\title{
OPTIMIZATION OF AN AIRFOIL'S PERFORMANCE THROUGH MOVING BOUNDARY CONTROL.
}

by

\author{
Sophie Dufresne
}

Thesis submitted to the Faculty of the

Virginia Polytechnic Institute and State University

in partial fulfillement of the requirenients for the degree of

Master of Science

in

Aerospace and Ocean Engineering

APPROVED:

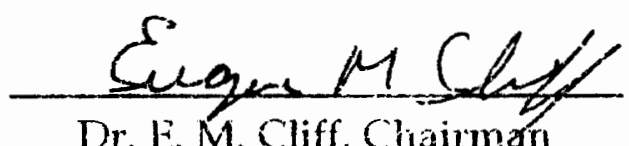

Dr. E. M. Cliff, Chairman
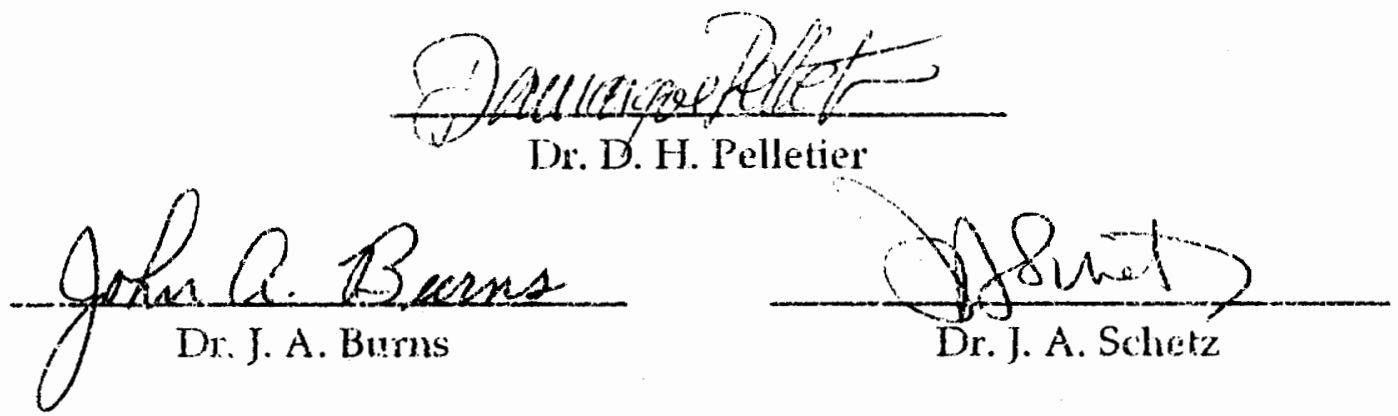

May 8,1993

Blacksburg, Virginia 


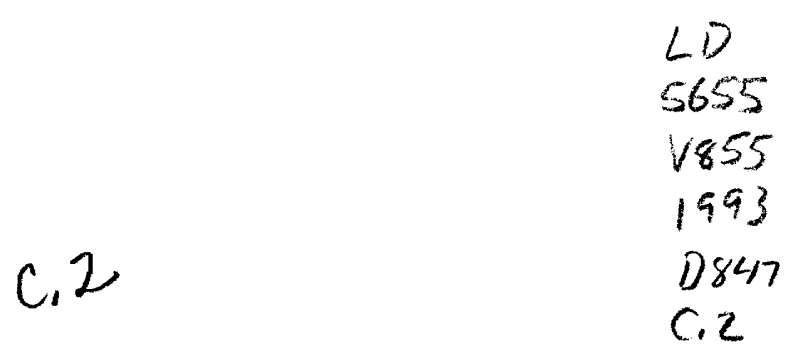




\title{
Optimization of an Airfoil's Performance \\ through Moving Boundary Control
}

\author{
by \\ Sophie Dufresne \\ Committee Chairman: Eugene M. Cliff \\ Aerospace and Ocean Engineering
}

(ABSTRACT)

The boundary-layer behavior over an airplane's wings is of great importance in take-off and landing of the airplane. If its angle of attack is increased past a critical value, the flow separates from the lifting surface, resulting in a drastic loss of lift and a major increase in drag. In response to this phenomenon, many mechanisms have been studied to control the boundary-layer. First neglected because of implementation difficulties of its application, moving wall boundary-layer control methods have mainly relied on experimental research. The moving wall concept is principally applied as a rotating cylinder protruding into the airfoil.

The purpose of this thesis is to provide a computational base to these experiments and to use mathematical tools of computational fluid dynamics and optimization to predict the optimum rotating speed of the cylinder, placed at the leading edge of the airfoil. For the sake of simplicity, we replace the airfoil by a flat plate with a wedge trailing edge. To model the incompressible viscous two-dimensional Navier-Stokes equations, the finite element method is applied on an unstructured two-dimensional mesh. An adaptive remeshing strategy utilized in conjunction with an error estimator controls the solution's accuracy. The aerodynamic forces acting on the total surface are computed from the finite element approximation. The ratio of the lift and the power required to move the flat plateairfoil and to rotate the cylinder forms the objective function to be optimized. A graph of the objective function versus the angle of attack is first constructed for several rotational speeds to provide a rough visual estimate of the optimum value for every angle of attack. Ultimately, an automatic optimization process provides the final solution. This results in the ideal rotational speed to be applied as the angle of attack varies. 


\section{Acknowledgments}

I would like to express my deep gratitude to Dr. E.M. Cliff and Dr. D.H. Pelletier who gave me the opportunity to complete my master's degree at Virginia Tech. Throughout the last two years, they have been very generous with their time and judicious advice and always ready to answer my questions. Here, I wish to give special thanks to Dr. Pelletier who, along with his expertise, provided the finite element program essential to the realization of this thesis. I would also like to thank Dr. J.A. Schetz and Dr. J.A. Burns who accepted to be part of my committee.

I wish to thank the Air Force Office of Scientific Research, who supported this research in parts under grant AFOSR-F49620-92-J-0078, and the F.C.A.R. of Québec (Fond Consolidé d'Aide à la Recherche) who granted me a two years scholarship.

I must mention the contribution of Jean-Francois Hétu whose help was very useful during the transfer from the VM/CMS system to a UNIX workstation. Also of great contribution was Benoit Ozell's visualization program (VU) with his explanations and advice. I wish to thank by the same occasion Vangelis Hytopoulos for many helpful and interesting discussions on fluid dynamics.

I am particularly grateful to my family who encouraged me to pursue my studies at Virginia Tech. Sincere thanks to Henry Brawner for his support throughout the course of this work and especially during these last difficult months. Finally, I wish to address many thanks to Marjukka Ollilainen, Horace and Jyothsna Sequeira and my caring roommates, Chiara Gratton Luc Lebel and Arend Van Wamelen, for their unfailing friendship and understanding in all circumstances. 


\section{TABLE OF CONTENTS}

\section{Chapter}

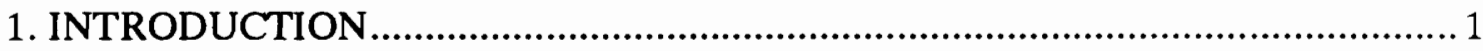

1.1 Historical Notes .......................................................................... 1

1.2 Moving Boundary-Layer Control Methods .........................................2 2

1.3 Rotating Cylinder as a Boundary-Layer Control ....................................2

1.4 Optimal Design of the Moving Boundary Control ..................................... 3

1.5 Optimization Study............................................................................ 4

2. FINITE ELEMENT METHODOLOGY .................................................. 5

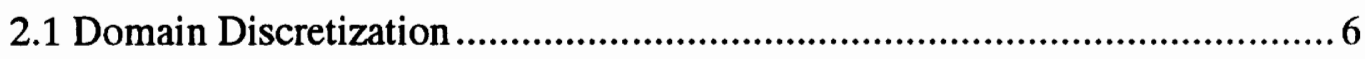

2.2 Finite Element Resolution................................................................. 7

2.3 Error Estimation................................................................................ 10

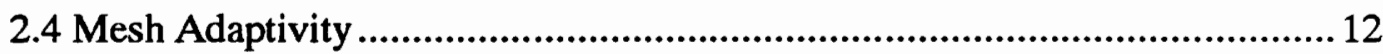

2.5 Aerodynamic Forces Computation.................................................... 14

3. FINITE ELEMENT METHOD APPLICATIONS ........................................... 18

3.1 Calibration Examples.............................................................. 18

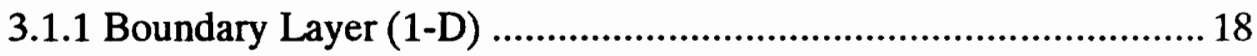

3.1.2 Two-Dimensional Half-Plates.............................................29

3.1.3 Two Concentric Rotating Cylinders ....................................... 41

3.2 Flat Plate Airfoil with Rotating L.E. Cylinder .....................................50

3.2.1 Discretization.................................................................50

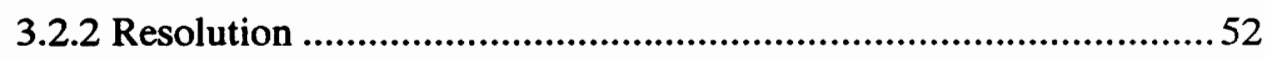

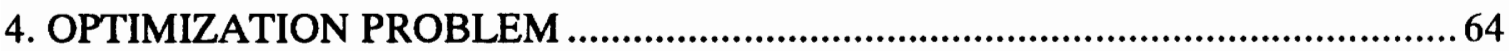

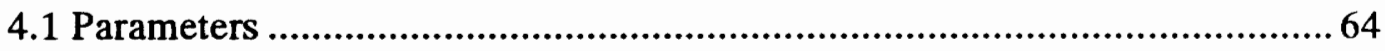

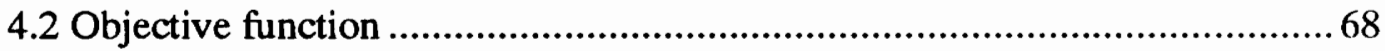

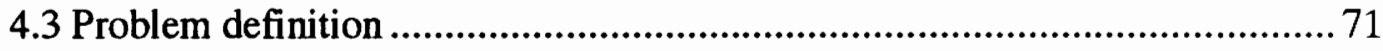

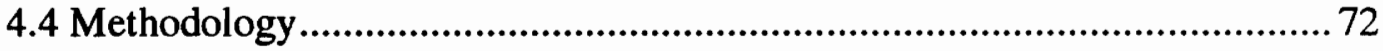

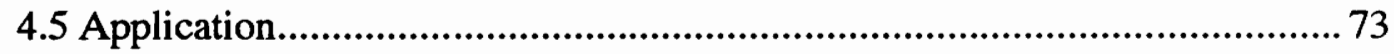




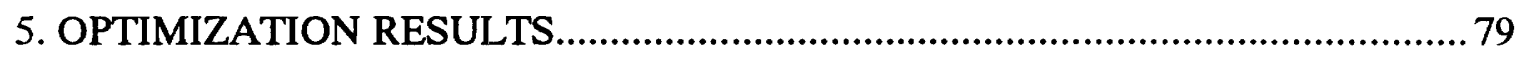

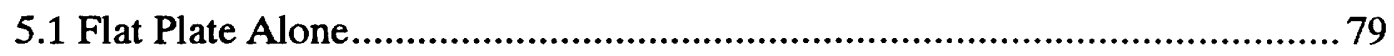

5.2 Flat Plate with Rotating Cylinder at Leading Edge ....................................... 81

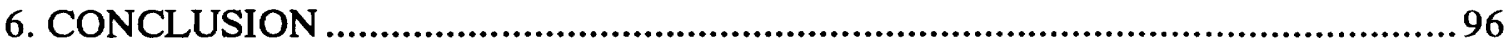

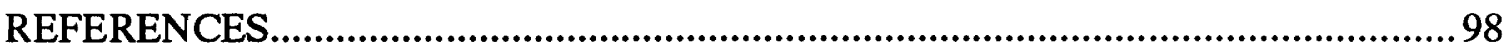

VITA 


\section{FIGURES}

2.1 Finite Element Adaptative Remeshing Algorithm.................................................5

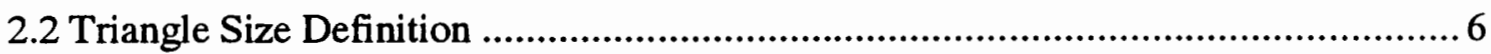

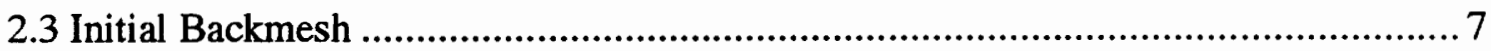

2.4 Crouzeix-Raviart element degrees of freedom .................................................... 9

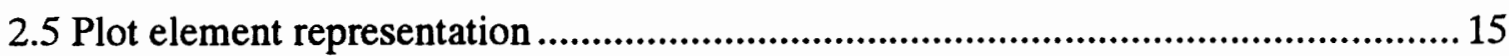

2.6 Geometrical transformation for Gauss rule (1-D)................................................... 16

3.1 Boundary-Layer Problem (1-D), Geometry …………................................................ 19

3.2 Boundary Layer Problem (1-D), First Backmesh ……...............................................20

3.3 Boundary-Layer Problem (1-D), Mesh Convergence ……….................................... 21

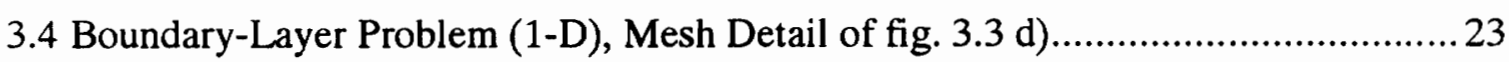

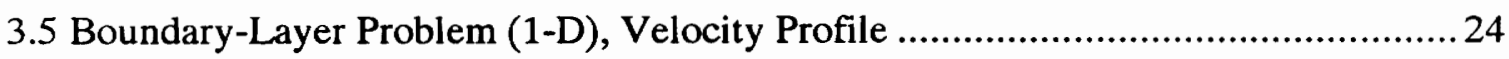

3.6 Boundary-Layer Problem (1-D), Pressure Distribution ............................................2. 25

3.7 Boundary-Layer Problem (1-D), Error Distribution a) Cycle (a) ................................26

b) Cycle (d) ..............................27

3.8 Two-Dimensional Half Plates, Geometry...............................................................29

3.9 Two-Dimensional Half-Plates, Computational region and boundary conditions.......29

3.10 Two-Dimensional Half-Plates, First Backmesh ...................................................... 30

3.11 Two-Dimensional Half-Plates, Mesh Convergence ................................................ 31

3.12 Two-Dimensional Half-Plate, $\mathrm{Re}=1200$, Velocity Distribution .............................. 32

3.13 Two-Dimensional Half-Plate, $R e=1200$, Pressure Distribution ...........................33

3.14 Two-Dimensional Half-Plate, $\mathrm{Re}=1200$, Error Distribution a) Cycle (a).............. 34

b) Cycle (b) ...............35

3.15 Transformation from Cartesian to Normal-Tangential System.................................36

3.16 Two-dimensional Half-Plate, $\operatorname{Re}=100$ a) Skin-Friction Coefficient

Distribution over the plate, b) Wake Centerline Velocity ................................................. 37

3.17 Two-dimensional Half-Plate, $\mathrm{Re}=400$ a) Skin-Friction Coefficient

Distribution over the plate, b) Wake Centerline Velocity .................................................38

3.18 Two-dimensional Half-Plate, $\mathrm{Re}=1200$ a) Skin-Friction Coefficient

Distribution over the plate, b) Wake Centerline Velocity .............................................39

3.19 Concentric Rotating Cylinders Problem, Geometry................................................. 41

3.20 Concentric Rotating Cylinders Problem, First Backmesh ....................................... 43

3.21 Concentric Rotating Cylinders Problem, Mesh Convergence .................................. 44 
3.22 Concentric Rotating Cylinders, Velocity Distribution .............................................45

3.23 Concentric Rotating Cylinders, Pressure Distribution ............................................46

3.24 Concentric Rotating Cylinders, Error Distribution a) Cycle (a), b) Cycle (b) ......... 47

3.25 Flat plate with Rotating L.E. Cylinder Problem ......................................................51

3.26 Flat Plate with Rotating L.E. Cylinder, Initial Backmesh ......................................52

3.27 Flat Plate with Rotating L.E. Cylinder, $\alpha=15^{\circ}$, Mesh Convergence .....................54

3.28 Flat Plate with Rotating L.E. Cylinder, $\alpha=15^{\circ}$, Mesh Detail of figure 3.27 c)......55

3.29 Flat Plate with Rotating L.E. Cylinder, Alpha $=15,(r \cdot w) / U=0.1$, Velocity

Distribution

3.30 Flat Plate with Rotating L.E. Cylinder, Alpha $=15,(r \cdot w) / U=0.1$, Pressure

Distribution

3.31 Flat Plate with Rotating L.E. Cylinder, Alpha $=15,(r \cdot w) / U=0.1$, Error

Distribution a) Cycle (a), b) Cycle (b), c) Cycle (c) in Figure 3.27.

3.32 Flat plate with L.E. Rotating Cylinder, Comparison with Modi \& Fernando's

Flat Plate with rotating L.E. Cylinder and non-rotating T.E. Cylinder.

4.1 Characteristic Quantities involved in the Flat Plate with Rotating Cylinder at

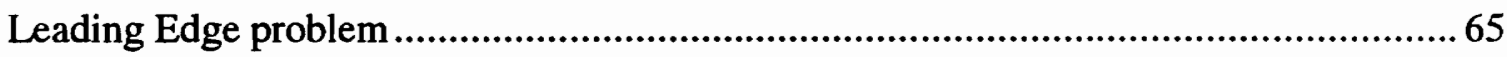

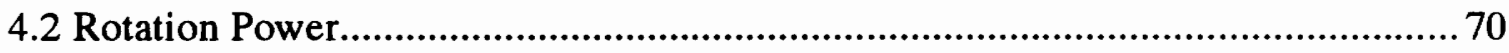

4.3 Initial Curve for the 20 Cylinder-Flat Plate System ................................................. 73

4.4 Iterative Optimization Process for the 20 Cylinder-Flat Plate System a) Second Iteration, b) Third Iteration, c) Corrected Third Iteration, d) Fourth Iteration, e) Fifth Iteration 74

5.1 Lift to Drag Ratio versus Angle of Attack for Flat Plate Alone 79

5.2 Lift and Drag coefficients versus Angle of Attack for Flat Plate Alone a) Lift Coefficient, b) Drag Coefficient 80

5.3 Finite Element Model, Lift Coefficient versus Angle of Attack 81

5.4 Experimental Results, Lift Coefficient versus Angle of Attack a) Modi \& Fernando, Flat Plate with Leading and Trailing Cylinders, b) Mokhtarian, Modi \& Yokomiso, Joukowsky Airfoil with Leading Edge Cylinder 82

5.5 Effect of Including Rotation Power in the Performance Ratio a) Lift to Drag ratio, b) Lift to Total Power ratio. 84 
5.6 Lift to Total Power ratio for various Rotation Speeds a) $0.0<(r \cdot \omega) / U<2.0$

b) $2.0<(r \cdot \omega) / U<4.0$

5.7 Optimization Problem, = 0 a) Non Symmetrical Domain b) Symmetrical

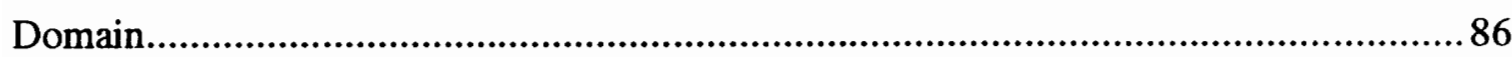

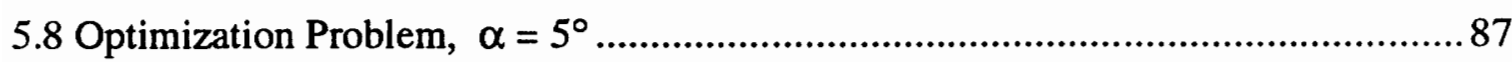

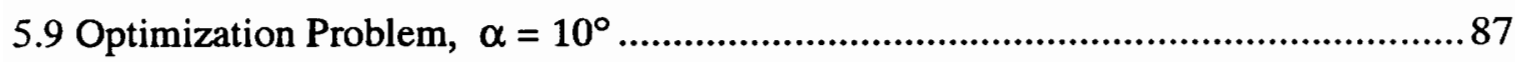

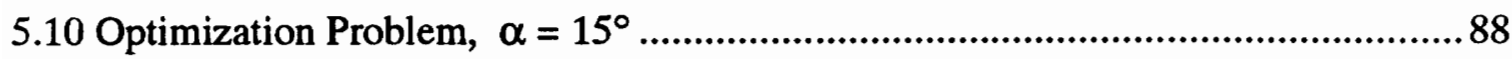

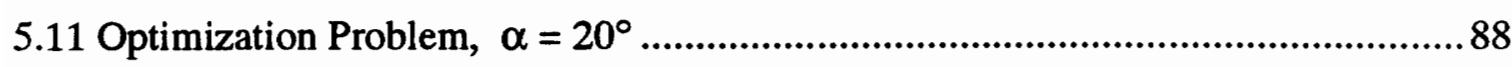

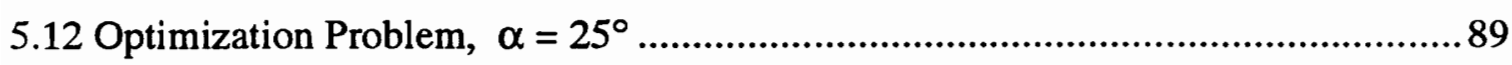

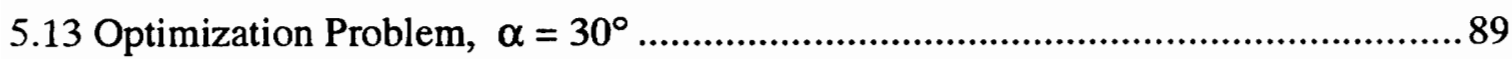

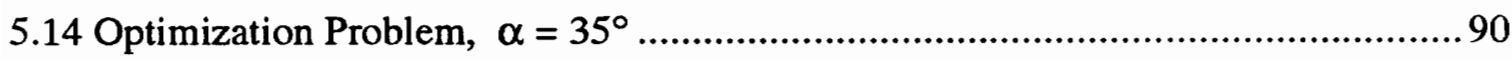

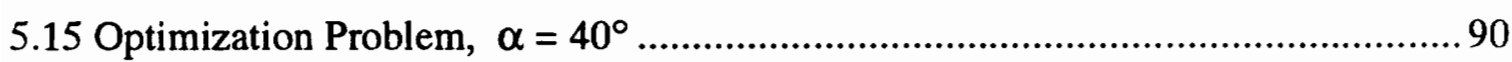

5.16 Base Flate Plate, Alpha = 15, Velocity Distribution ...........................................

5.17 Flate Plate with Rotating L.E. Cylinder, Alpha $=15,(r \cdot w) / U=1.63=$

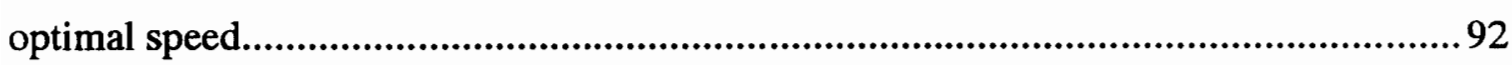

5.18 Optimal Cylinder Angular Speed versus Angle of Attack.....................................94 


\section{TABLES}

3.1 Boundary-Layer problem, Adaptivity Strategy ......................................................22

3.2 Comparison between analytical results and f.e.m. solution for the one-

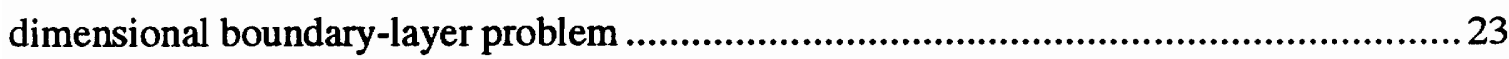

3.3 Characteristics of the domain for the two-dimensional half-plates ...........................29

3.4 Two-Dimensional Half-Plates, size function for the initial backmesh..........................30

3.5 Concentric Rotating Cylinders, Adaptivity Strategy ....................................................49

3.6 Comparison between analytical results and f.e.m. solution for the two co-axial

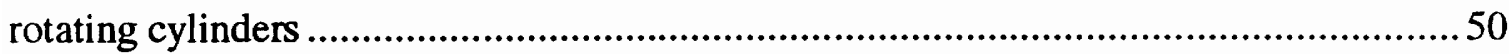

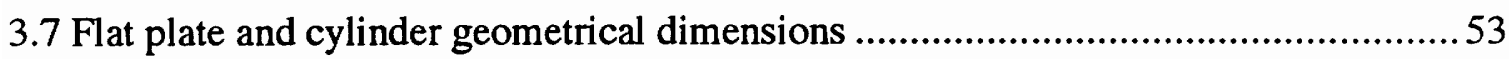

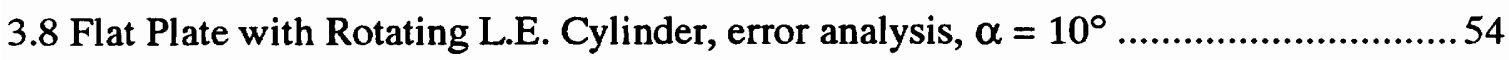

3.9 Flat Plate with Rotating L.E. Cylinder, Adaptivity Strategy .....................................56

3.10 Flat Plate with Rotating L.E. Cylinder, $\alpha=15^{\circ}, \mathrm{Re}=1000$, Aerodynamic

Forces 62

3.11 Flat Plate with Rotating Cylinder at Leading Edge and Non-Rotating Cylinder at Trailing Edge, $\alpha=15^{\circ}, \operatorname{Re}=30000$, Aerodynamic Coefficients …........................... 77

4.1 Iterative Optimization Process for the $20^{\circ}$ angle case .............................................94

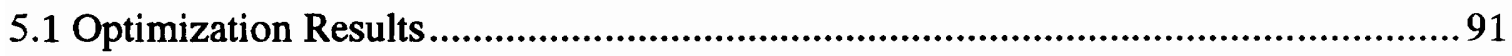




\section{CHAPTER 1}

\section{INTRODUCTION}

\subsection{Historical Notes}

Throughout history, one of the greatest dream of human kind was to be able to fly like birds. From legendary stories, such as the myth of Daedalus and Icarus, through speculations of artists like Leonardo da Vinci (1452-1519), and to the first aerodynamic experiments, human flight remained in the realm of imagination. Preceded by many unsuccessful bird imitating models, the rigid airplane principle was first introduced by $\mathrm{Sir}$ George Caley (1773-1857) in 1799. Little theory about fluid mechanics was known at the time except for Newton's theory of air resistance (Philosophiae Naturalis Principia Mathematica,1726) and the more rational findings of the mathematician D'Alembert (Essai d'une nouvelle théorie de la résistance des fluides, 1752). Throughout the nineteenth century, the mathematical theory of fluid dynamics grew but was of little use to the practical application of flight. The early aerodynamicists were especially interested in finding the most efficient wing shape. Wind tunnel tests conducted on several wing profiles revealed the superiority of curved surfaces over flat ones. However, no theory could explain the fact that curved surfaces produced lift at zero angle of attack. The theory of lift, based on circulation and derived from the so-called Magnus effect (Lanchester•1878-1946, Kutta•1867-1944 and Joukowski•1847-1921), appeared around the same time the Wright brothers accomplished their first successful powered flight in 1903. But it is only with the introduction of the boundary layer concept by Prandtl (1904) that aeronautical design found its mathematical base.1

Prandtl showed that, for the problem of a body moving through a fluid of small viscosity such as air or water, viscous forces were significant only in a thin layer adjacent to the surface of the body, the boundary-layer. This viscosity causes a shear stress at the surface which slows down the fluid particles in the boundary-layer. If the presence of an adverse pressure gradient in the flow retards the fluid particles sufficiently, a phenomenon called separation occurs, causing reverse flow downstream of the separation point3. Two major consequences of a separating flow over a lifting surface are a drastic loss of lift and a major increase in drag2. Prandtl showed by several experiments, as he introduced the concept of boundary-layer and the mechanism of separation, that the boundary-layer could 
be controlled in order to postpone separation. Such a technique of control is called boundary-layer control (BLC).

\subsection{Moving Boundary-Layer Control Methods}

Boundary-layer control is used in many applications involving fluid flow, such as aeronautic and hydronautic vehicles, diffusers, compressors and windmills For the particular case of an airplane, boundary-layer control can lead to greater maneuverability, longer range, shorter take-off and landing runs. Many boundary-layer control methods have been studied, including shaping, wall suction, wall motion, wall heating/cooling, wave cancellation, turbulators, streamlining and the moving wall effect3.

Of all these methods, the moving wall boundary-layer control, derived from the so-called Magnus effect, has received relatively little attention. The effect of a deviation on the trajectory of a flying ball when set in rotation has long been observed and used (in ballistics and sports) before a mathematical theory could explain the phenomenon. Magnus (1852) first recognized the presence of an aerodynamic force produced by an unsymmetrical pressure distribution due to the Bernouilli effect. Around the same time, Rayleigh (1877) studied the case of a rotating cylinder in a flow stream and later, Flettner (1924) applied this concept to a ship where he replaced the sail by rotating cylinders4. Introduced as a possible boundary-layer control device by Prandtl about 1907, followed by Goldstein in 1934, the first experiments of an aeronautical application were conducted in Holland (Wolff and Koning, 1926) and in France (Favre, 1936)s. Favre's experiment replaced the upper part of an airfoil with an endless band of silk in motion with the fluid. $\mathrm{He}$ obtained a significant increase in lift at very high angle of attack but because of the mechanical difficulty of its implementation, the idea was abandoned.

\subsection{Rotating Cylinder as a Boundary-Layer Control}

About 25 years later, in 1961, Alvarez-Calderon tried another form of the moving wall boundary control by putting a rotating cylinder at the leading edge of an airfoil's flap for a V./S.T.O.L.-type aircraft. Flight tests of the system showed great improvement in handling qualities and control characteristics of the aircraft. This configuration was tested again with success on OV-10A-type aircraft at NASA's Ames Research Center, s, in the seventies. In a more scientific approach, Tennant et al. tested different possibilities for the rotating cylinder-airfoil combination. They compared analytical models to experimental 
results for a leading edge rotating cylinder mounted on a wedge shaped flap and a trailing edge rotating cylinder on a symmetrical airfoil. Also studied was the boundary-layer growth on moving surface taking into account gap effects $10,11,12$.

More recently, Modi, Mokhtarian et al. studied several configurations of one or more rotating cylinders mounted on an airfoil. They found that the leading edge cylinder provided more benefit than the trailing edge cylinder ${ }^{13}$, which spoils the configuration with an additional gap ${ }^{14}$. However, the trailing edge cylinder acts effectively as a flap, increasing the lift at the smaller angles of attack. The scooped leading edge cylinder configuration was found to be of limited advantage since it only affects the flow at low rotational speeds ${ }^{15}$. The leading edge cylinder effect results in a large suction peak at the nose which still isn't sufficient enough to prevent separation downstream if a high angle of attack causes an important adverse pressure gradient. The most promising configuration seems to be a cylinder located at the upper surface of the airfoil as it helps to reduce separation further downstream ${ }^{16}$. Using more than one cylinder is not the most economical solution since its mechanical implementation is more complicated and more power is required to rotate the cylinders.

\subsection{Optimal Design of the Moving Boundary Control}

In the previous work on the effects of a rotating cylinder as a high lift device on the aerodynamic performances of an airfoil, results have mainly been obtained empirically on specific configurations. Several tests have been performed on many arrangements that resulted in no definite indication of the parameters to use in order to generate the optimal aerodynamic characteristics. Some of these parameters could be listed as:

- Cylinder diameter

- Cylinder-airfoil gap

- Cylinder location on the airfoil

- Cylinder rotational speed

Using the powerful capacities of the computer, models of different configurations (type of airfoil, location and dimensions of the cylinder, free stream velocity, etc.) can be created without great difficulty or resources. Although the "real world" situation is greatly simplified, a relatively good idea of the actual phenomenon can be deduced from a numerical model. Furthermore, a numerical implementation allows one to easily 
manipulate the above parameters in order to study their effects. Optimization techniques supply a powerful way to determine the parameters that will generate the optimal aerodynamic performances of a given configuration.

\subsection{Optimization Study}

Based on the previous experimental results, the present study will consider the leadingedge configuration which provides a good compromise between efficiency and simplicity. As pointed earlier, there exists no mechanism that can theoretically predict the optimum parameters of a moving wall boundary-layer control. Mokhtarian and Modi1 used a numerical surface singularity approach and a finite difference boundary layer scheme accounting for viscous effects, both combined in an iterative procedure to solve for the flow past an airfoil with a leading-edge cylinder. They obtained fairly good agreement with a wind tunnel test, although they suggest a resolution of the full Navier-Stokes equations to obtain better accuracy. The finite element approach provides a powerful way to model such equations. We will use this method combined with an adaptative remeshing strategy. An unstructured two-dimensional mesh is used to model the leading-edge cylinder configuration.

The following chapter outlines the methodology of the finite element resolution for the Navier-Stokes equations. Also in the second chapter, we describe the computation of the aerodynamic forces from the finite element solution. The third chapter shows applications of the methodology. Three simple examples provide a way to calibrate the method and are followed by the flow resolution of the airfoil with rotating cylinder at leading edge. To simplify the calculations, the airfoil is replaced by a flat plate with a wedge trailing edge. A description of the optimization problem comes in the fourth chapter where we summarize the optimization procedure for a fixed angle of attack. Finally, the fifth chapter presents the results of the optimization study for the flat plate-airfoil with leading edge rotating cylinder. 


\section{CHAPTER 2}

\section{Finite Element Methodology}

To model the flow around the high lift wing, the assumption that little variations exists in the spanwise direction is postulated. Therefore, a two-dimensional simplification can be applied and the domain results in a rectangular area surrounding the wing section. The flow is solved by an adaptive finite element method.

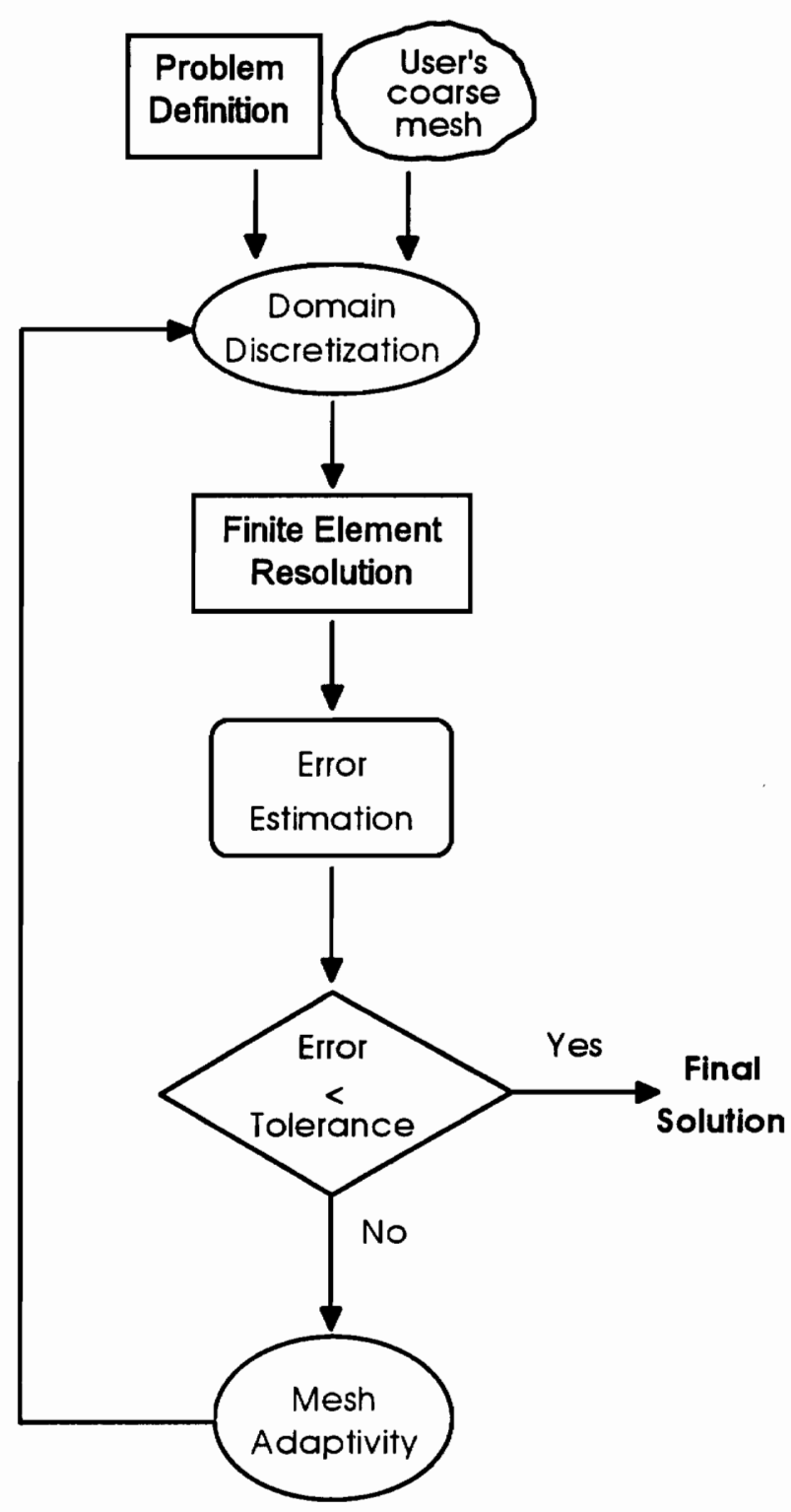

Figure 2.1 Finite Element Adaptive Remeshing Algorithm 
The general scheme consist of four steps:
1. Domain discretization
2. Finite Element resolution
3. Error Estimation
4. Mesh Adaptivity

The relation between these four steps can be schematically represented by figure 2.1. This chapter covers briefly each phase of the procedure.

\subsection{Domain Discretization}

To construct a finite element problem, we must discretize the problem's computational area into many sub-domains and define its characteristic on the grid formed by these subdomains. The problem characteristics consist in:

- flow description (two-dimensional, steady, laminar, no heat transfer.)

- geometry (coordinates, curves, areas)

- boundary conditions (Dirichlet, Neumann)

- mesh attributes (type of element,...)

- resolution parameters (initial conditions, convergence criteria,...)

A unstructured triangular grid is used to mesh the domain of interest because these grids provide the flexibility to handle complex geometries. This grid is generated by an advancing front mesh generator ${ }^{29}$.

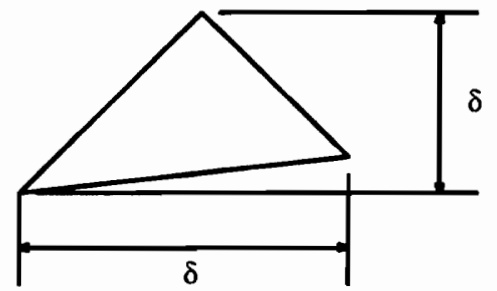

Figure 2.2 Triangle Size Definition

The size of the triangles (see figure 2.2) to be generated are linearly interpolated on the backmesh were point values for the elements size are defined on each node. This allows flexibility to force more elements where the flow resolution requires more accuracy and fewer elements where it is not needed. The geometry is defined within areas which themselves are delimited with curves. A curve is defined by a set of geometrical points. The mesh generator starts the triangulation from the boundaries and gradually advances toward the center of the area. The triangulation process starts at one point and generates 
the elements successively along the boundaries. For this reason, the grids generated by the mesh generator won't generally be symmetric. The backmesh can be made of very few elements but must cover entirely the computational area, as shown in figure 2.3.

The initial backmesh is a coarse mesh made of linear (three nodes) triangles and is constructed manually. The mesh generator produces the first grid according to the elements size defined on the backmesh. The size function $\delta$ is piecewise linear. It is only continuous within one element and is constructed from three nodal values defined on that element. The grid generated on this framework should be able to capture roughly the domain characteristics.

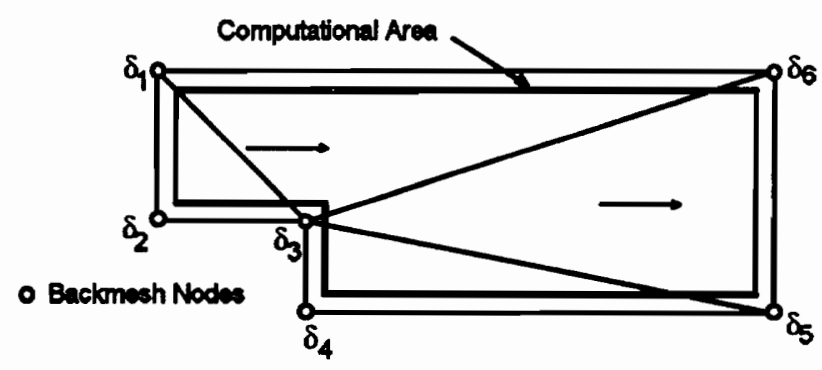

Figure 2.3 Initial Backmesh

Once an approximate solution is obtained with the initial backmesh, we compute the error distribution on the grid. If the solution is not accurate enough, a new grid density function is generated to construct a mesh that is more "adapted" to the problem. The algorithm then begin a second iteration. The size function is applied on the first mesh which becomes the backmesh for the second iteration. This process is repeated until a satisfactory degree of accuracy is obtained. These ideas are described in more details in section 2.4.

\subsection{Finite Element Resolution}

For Reynolds numbers beyond a critical value, the developing boundary-layer would become turbulent. While it is possible to directly simulate such flows, the mathematical model must account for unsteadiness and numerical approximations would require very fine spatial discretization. The more usual procedure, based on Reynolds averaging, requires some explicit model for the turbulence-induced stresses. In this study, we consider only the case of laminar flow. Since our concern here is mainly the take off and landing flight situations, a low speed flow is understood. Thus, the flow is assumed to be 
incompressible. Isothermal and steady state conditions are implied. Accordingly, the governing equations used to model this flow are the isothermal Navier-Stokes equations with the Boussinesq approximation 19,20:

$$
\begin{array}{lll}
\text { Conservation of mass } & \nabla \cdot \mathbf{u}=0 & \text { in } \Omega \\
\text { Conservation of momentum } & \rho \mathbf{u} \cdot \nabla \mathbf{u}=-\nabla p+\nabla \cdot \tau+\mathbf{f} \quad \text { in } \Omega
\end{array}
$$

where $\tau$ is the viscous stress tensor is described as

$$
\tau=2 \mu \dot{\gamma}
$$

for a Newtonian fluid. The strain tensor $\dot{\gamma}$ is defined as

$$
\dot{\gamma}(\mathbf{u})=\frac{1}{2}\left\{\nabla \mathbf{u}+(\nabla \mathbf{u})^{T}\right\} .
$$

Finally, to complete the problem description, boundary conditions are applied on $\partial \Omega$ :

$$
\begin{array}{lr}
\mathbf{u}=\mathbf{u}_{0} & \text { on } \Gamma_{\boldsymbol{u}} \\
2 \mu \dot{\gamma} \cdot \mathbf{n}-p \mathbf{n}=\hat{\mathbf{t}} & \text { on } \Gamma_{\hat{t}},
\end{array}
$$

where $\mathbf{u}_{0}$ and $\hat{\mathbf{t}}$ are specified fuctions. The boundary $\partial \Omega$ is divided between $\Gamma_{u}$ and $\Gamma_{\hat{t}}$ such that

$$
\begin{aligned}
& \partial \Omega=\Gamma_{u} \cup \Gamma_{\hat{t}} \\
& \Gamma_{u} \cap \Gamma_{\hat{i}}-\varnothing
\end{aligned}
$$

A variational method 18 can be applied to solve the system of equations once the differential equations have been cast in a variational (or weak) form19,20:

$$
\begin{aligned}
& (\mathbf{q}, \nabla \cdot \mathbf{u})=0 \\
& (\boldsymbol{\rho} \mathbf{u} \cdot \nabla \mathbf{u}, \mathbf{v})+a(\mathbf{u}, \mathbf{v})-(p, \nabla \cdot \mathbf{v})=(\vec{f}, \mathbf{v})+\langle\hat{\mathbf{t}}, \mathbf{v}\rangle_{\Gamma}
\end{aligned}
$$

where

$$
a(u, v) \equiv \int_{\Omega} 2 \mu \dot{\gamma}(u): \dot{\gamma}(v) d \Omega
$$

and $\mathbf{v}$ and $\mathbf{q}$ are appropriate test functions such that

$$
\begin{aligned}
& \mathbf{v} \in V=\left\{\mathbf{v} \in\left(H^{1}(\Omega)\right)^{2} \mid \mathbf{v}=0 \text { on } \Gamma_{u}\right\} \\
& \mathbf{q} \in Q=L^{2}(\Omega)
\end{aligned}
$$


and $(;)$ is the inner product defined on $\mathrm{L}^{2}(\Omega)$.

These equations are discretized by the seven node Crouzeix-Raviart element (enriched quadratic velocity interpolation and discontinuous linear pressure approximation)19:

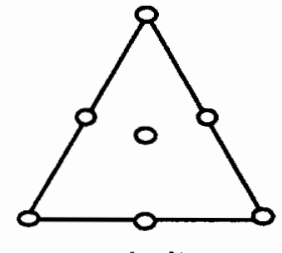

velocity

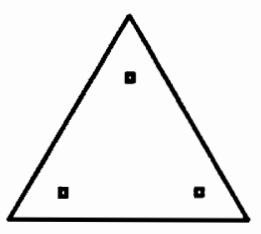

pressure

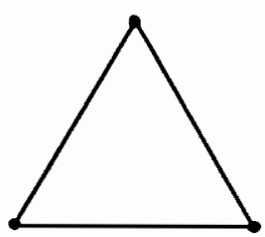

geometry

Figure 2.4 Crouzeix-Raviart element degrees of freedom

The Galerkin variational formulation is used. Thus the test functions are also equal to the interpolation functions associated with the element. Inspired from the Stokes linear case where a quadratic functional exists and is represented by

$$
\begin{aligned}
& I(\mathbf{u}, p)=\hat{I}(\mathbf{u})-(p, \nabla \cdot u), \\
& \hat{I}(\mathbf{u}) \equiv \frac{1}{2} a(u, u)-(f, u)-\langle\hat{t}, u\rangle_{\Gamma},
\end{aligned}
$$

we can easily see that the problem can be formulated as minimizing $\hat{\mathrm{I}}(\mathbf{u})$ under the constraint $\nabla \cdot \mathbf{u}=0$, taking $p$ as a Lagrange multiplier. If we apply the penalty method on this problem by replacing the second right hand side term in (2.13) with the penalty term $\varepsilon(\nabla \cdot \mathbf{v}, \nabla \cdot \mathbf{u})$, we eliminate pressure as a degree of freedom. This can also be applied to the Navier-Stokes non-linear case since the convection terms do not involve the pressure21. Since we want to solve the equations for both velocity and pressure, we use a modified version of the penalty method. This modified version combines the Lagrangian formulation with the penalty method and is called augmented Lagrangian or Uzawa formulation. Reformulating the variational form (2.9) in the Uzawa formulation, we get:

$$
\begin{gathered}
\Rightarrow(\rho \mathbf{u} \cdot \nabla \mathbf{u}, v)^{n+1}+a(u, v)^{n+1}-(\vec{f}, v)^{n+1}-\langle\hat{t}, v\rangle_{\Gamma}^{n+1}+\varepsilon(\nabla \cdot v, \nabla \cdot u)^{n+1}=\left(p^{n}, \nabla \cdot v\right) \\
|\nabla \cdot v| \leq \text { stolerance } \underset{\text { yos }}{\rightarrow} \text { stop } \\
\Leftarrow p^{n+1}=p^{n} \downarrow \varepsilon(\nabla \cdot v, \nabla \cdot u)
\end{gathered}
$$


In order to eliminate the pressure degree of freedom from the system and still solve for it, the problem is formulated as an iterative process. An initial guess is given for the pressure value $(n=0)$ with which we solve for $\mathbf{u}$ the upper equation in (2.15). Subsequently, we check the continuity constraint requirement. If the constraint is not satisfied within a certain tolerance, we compute a new estimate for the pressure and feed it back into the equation. The process is repeated until the constraint conforms to the specified tolerance. Applied to a finite element problem, the Augmented Lagrangian formulation reduces its total number of degrees of freedom by one third. In addition, the elimination of two velocity and two pressure d.o.f. at the centroid by a condensation of the bubble function in the Crouzeix-Raviart element finally reduces the system from $66 \%$ to $44 \%$ of its total $\operatorname{size}^{2}$.

The weak form of the Navier-Stokes equations in (2.14) is obtained on each element. All elementary matrices are then assembled in a compacted skyline format to save memory. The system of nonlinear equations is linearized by either the successive substitution or the Newton-Raphson method. The choice of the method to use relies on the following consideration: the method of successive substitution has a large radius of convergence but has also, for many problems, a slow rate of convergence and the Newton-Raphson method has a quadratic rate of convergence but a much smaller convergence radius. The strategy found to be the most effective is to use the successive substitution method for the first few iterations and then switch to Newton-Raphson method until convergence is reached. Each iteration of the Uzawa formulation requires the resolution of the assembled system by LU decomposition.

\subsection{Error Estimation}

The a posteriori error estimators used by the adaptive module fall in the the following categories:

- Exact error method;

- Projection method;

- Element residuals method;

- Local PDE problem method;

- Internal-external approximation method.

When the exact solution is not available, we must use one the four last methods. For the Navier-Stokes equations, the most effective methods were found to be the projection and 
the local PDE problem methods ${ }^{19}$. The error estimator chosen here is the projection method since it treats the problem more globally than the local PDE problem method and requires a lower degree for the approximation polynomials. To measure the error, an appropriate norm must be selected. A series of norms or seminorms are available to measure velocity, pressure, and temperature in a scalar or vectorial form, as required (see ref. 19, p.57). The Stokes norm will be applied here since it involves both velocity and pressure:

$$
\|(\mathbf{u}, \mathbf{p})\|_{\mathrm{S}, \Omega}=\left\{\|\mathbf{u}\|_{E, \Omega}{ }^{2}+\|p\|^{2}{ }_{0, \Omega}\right\}^{\frac{1}{2}},
$$

where the first term in the right hand side is called the energy norm and is defined as:

$$
\|\mathbf{u}\|_{E, \Omega}^{2}=\int_{\Omega} 4 \mu^{2} \dot{\gamma}(\mathbf{u}): \dot{\gamma}(\mathbf{u}) \mathrm{d} \Omega
$$

and the second right hand side term is the norm $\mathbf{L}^{2}$ of the pressure:

$$
\|p\|_{0, \Omega}^{2}=\int_{\Omega}|p|^{2} d \Omega
$$

From this definition, the error norm for velocity and pressure over an element is described as:

where

$$
\left\|e^{u, p}\right\|_{K}=\left\{\int_{K}\left[\mu^{2}\left(\nabla e^{u}+\nabla\left(e^{u}\right)^{T}\right)^{2}+\left|e^{p}\right|^{2}\right] d x\right\}^{\frac{1}{2}}
$$

and $\quad e^{p}=p-p_{h}$

$$
\nabla e^{u}=\frac{\partial e^{u}}{\partial x}=\frac{\partial u}{\partial x}-\frac{\partial u_{h}}{\partial x}
$$

The projection method, introduced by Zhu and Zienkiewicz, considers the fact that the exact solution has continuous derivatives while the approximate or finite-element derivatives are discontinuous. The idea is to estimate the exact derivatives with continuous functions which are obtained by projecting the discontinuous $\partial u_{h} / \partial x$ and $p_{h}$ in a leastsquare space of continous functions, say the velocity interpolation functions:

$$
\left[\sum_{k \in \mathbf{t}} \int_{K} \phi_{j} \phi_{i} d \mathbf{x}\right]\left\{\frac{\partial \tilde{\mathbf{u}}_{h}}{\partial \mathbf{x}}\right\}_{i}=\left\{\sum_{k \in \mathrm{t}} \int_{K} \phi_{j} \frac{\partial \mathbf{u}_{h}}{\partial \mathbf{x}} d \mathbf{x}\right\}
$$


and

$$
\left[\sum_{k \in \mathbf{t}} \int_{K} \phi_{j} \phi_{i} d \mathbf{x}\right]\left\{\tilde{p}_{h}\right\}_{i}=\left\{\sum_{k \in t} \int_{K} \phi_{j} p_{h} d \mathbf{x}\right\}
$$

The $\phi_{i}$ are the quadratic velocity interpolation functions of the Crouzeix-Raviart element. Assembling the global system and solving for the nodal values $\left\{\frac{\partial \tilde{\mathbf{u}}_{h}}{\partial \mathbf{x}}\right\}_{i}$ and $\left\{\tilde{p}_{h}\right\}_{i}$, we can compute the Stokes norm for the error estimated over the domain knowing that

$$
\nabla e^{\mathbf{u}} \cong \frac{\partial e_{h}^{\mathbf{u}}}{\partial \mathbf{x}}=\frac{\partial \tilde{\mathbf{u}}_{h}}{\partial \mathbf{x}}-\frac{\partial \mathbf{u}_{h}}{\partial \mathbf{x}}
$$

and

$$
e^{p} \cong \tilde{p}_{h}-p_{h}
$$

For more information on the error estimators, consult ref. 19, chap.3.

\subsection{Mesh Adaptivity}

From the estimated error distribution over the domain, a new improved mesh is generated. Several strategies exists to adapt the discretization for a better solution:

- $\quad h$-method: mesh refinement;

- $r$-method: relocation of nodes in an existing mesh;

- $\quad p$-method: enrichment of space containing the interpolation functions;

- combined methods: combination of the methods above.

The method used here is the mesh refinement technique for its flexibility, simplicity and its effectiveness on an unstructured mesh. A mechanism called the transition operator deduces from the error estimation the size function that will lead to an optimal mesh in the sense that the error is uniformly distributed on the mesh. Two different transition operators may be used to construct the new size function. The difference between the two operator lies in the definition of the total admissible error $\mathrm{e}_{\mathbf{T}}$ over the domain. The reduction operator tends to reduce the error by a given percentage $\xi$ while the fixed target operator seeks a given value of the relative error $\bar{\eta}$ :

reduction

$$
\mathrm{e}_{\mathrm{T}}=\bar{\eta}\left\|\mathrm{u}_{\mathrm{h}}\right\|_{\Omega}
$$




\section{fixed target}

$$
e_{T}^{n+1}=\xi\left\|e_{h}^{n}\right\|_{\Omega}
$$

so that

$$
\bar{\eta}=\frac{\left\|e_{h}\right\|_{\Omega}}{\left\|u_{h}\right\|_{\Omega}} .
$$

$\left\|e_{h}\right\|_{\Omega}$ and $\left\|u_{h}\right\|_{\Omega}$ are respectively the approximate error and velocity norms over the domain. As pointed out in the previous section, the Stokes norm is used for this problem. For the mesh to be optimal, all elements must have the same error. The admissible error $\overline{\mathrm{e}}$ over an element $k$ can be stated as follows:

$$
\bar{e}=\frac{e_{T}}{\sqrt{N}}
$$

where $\mathbf{N}$ represents the total number of elements in the mesh. On the other hand, we can relate the error over an element $k$ to its size $h$ with a constant of proportionality $C$, using the element's asymptotic rate of convergence $\alpha$ :

$$
\|e\|_{k} \cong C h_{k}^{\alpha}
$$

This relation can also be applied to the admissible error over an element $k$ and its corresponding optimal size $\delta_{k}$ :

$$
\bar{e} \cong C \delta_{k}^{\alpha} .
$$

Using (2.29) and solving for $C$ in (2.30) we get for (2.31):

$$
\frac{e_{T}}{\sqrt{N}}=\frac{\|e\|_{k}}{h_{k}^{\alpha}} \delta_{k}^{\alpha} .
$$

Finally, replacing equations (2.25) and (2.36) in (2.32), we obtain the optimal size for each element of the domain:

reduction

$$
\delta_{k}=\left[\frac{\bar{\eta}\left\|\mathbf{u}_{h}\right\|_{\Omega}}{\sqrt{N}\|e\|_{k}}\right]^{1 / \alpha} h_{k}
$$




\section{fixed target}

$$
\delta_{k}=\left[\frac{\xi\left\|e_{h}\right\|_{\Omega}}{\sqrt{N}\|e\|_{k}}\right]^{1 / \alpha} h_{k}
$$

Depending upon the choice of transition operator, equations (2.33) or (2.34) is applied on the nodes of each element $k$ of the mesh. This mesh then becomes the backmesh for the next iteration (when the type of element used to discretize the domain has more than three nodes, the elements of the actual mesh are cut into linear triangles to meet the requirements of the mesh generator, cf. section 2.1). The mesh generator constructs a new grid according to this backmesh and a new solution is computed on this improved grid. We may perform another error analysis on this new solution and begin a new iteration (see figure 2.1). This iterative process is repeated until we obtain a good level of accuracy for the resolution of the Navier-Stokes equations. To solve these equations, each iteration uses the previous solution as initial conditions and the first iteration is solved with zero initial conditions.

\subsection{Aerodynamic Forces Computation}

The lift and drag are defined respectively as the vertical and horizontal force generated by the fluid flow:

$$
\mathbf{F}=\left\{\begin{array}{l}
F_{x} \\
F_{y}
\end{array}\right\}=\int_{\Gamma}(\tau \cdot \mathbf{n}) d \Gamma=\left\{\begin{array}{l}
L \\
D
\end{array}\right\}
$$

For a viscous incompressible Newtonian fluid, the stress tensor $\tau$ is defined as:

$$
\begin{aligned}
{\left[\begin{array}{ll}
\tau_{x x} & \tau_{x y} \\
\tau_{x y} & \tau_{y y}
\end{array}\right] } & =\mu\left[\nabla \mathbf{u}+(\nabla \mathbf{u})^{T}\right]-p[I] \\
& =\left[\begin{array}{ll}
-p+2 \mu \frac{\partial u}{\partial x} & \mu\left(\frac{\partial u}{\partial y}+\frac{\partial \nu}{\partial x}\right) \\
\mu\left(\frac{\partial u}{\partial y}+\frac{\partial \nu}{\partial x}\right) & -p+2 \frac{\partial \nu}{\partial y}
\end{array}\right],
\end{aligned}
$$


where $u$ and $v$ are the Cartesian components of the velocity vector.

Thus, combining (2.35) and (2.36), we obtain for the lift and drag the following expression:

$$
\left\{\begin{array}{l}
L \\
D
\end{array}\right\}=\int_{\Gamma}\left[\begin{array}{ll}
\tau_{x x} & \tau_{x y} \\
\tau_{x y} & \tau_{y y}
\end{array}\right]\left\{\begin{array}{l}
n_{x} \\
n_{y}
\end{array}\right\} d \Gamma=\int_{\Gamma}\left\{\begin{array}{l}
\tau_{x x} n_{x}+\tau_{x y} n_{y} \\
\tau_{x y} n_{x}+\tau_{y y} n_{y}
\end{array}\right\} d \Gamma,
$$

where $n_{x}$ and $n_{y}$ expresses the Cartesian decomposition of the vector $\mathbf{n}$ that is normal to the body surface, and $\Gamma$ is the total boundary formed by the cylinder and the airfoil.

The finite element solution obtained from the solver gives the pressure distribution $p$ over the element and the estimated values for the two velocity component $u$ and $v$ at each node of the mesh. However, in order to compute the stress tensor from the f.e.m. approximation, we need to know the derivatives of $u$ and $v$ in addition to the pressure. Those derivatives can be easily computed from the finite element approximation on an element:

$$
\mathbf{u}(\mathbf{x})=\sum_{i=1}^{n d p} N_{i}^{u}(\mathbf{x}) \mathbf{u}_{i} \quad \Rightarrow \quad \frac{d \mathbf{u}}{d \mathbf{x}}(\mathbf{x})=\sum_{i=1}^{n d p} \frac{d N_{i}^{u}}{d \mathbf{x}}(\mathbf{x}) \mathbf{u}_{i}
$$

where the $N_{i}^{u}(x)$ are the interpolation functions associated with the velocity degrees of freedom (see section 2.2), and the $u_{i}$ are the nodal values of velocity. The equations necessary to construct the objective function only involve line integrals along the airfoil and cylinder curves.

Those curves are divided into one-dimensional elements, called plot elements, which are coincident with the adjacent side of the corresponding triangular element:

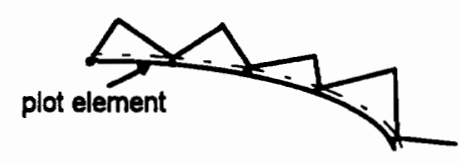

Figure 2.5 Plot element representation 
Referring to Gauss's numerical integration ruless for the one-dimensional case, we only need to compute the stress tensor at specific points, called gauss points, on this boundary. The total integral is decomposed to a sum of sub-integrals, performed over each plot element. Gauss's rule is therefore applied individually on each plot element:

$$
\left\{\begin{array}{l}
F_{x} \\
F_{y}
\end{array}\right\}=\sum_{e=1}^{N} \sum_{i=1}^{n_{g}}\left[\begin{array}{ll}
\tau_{x x} & \tau_{x y} \\
\tau_{x y} & \tau_{y y}
\end{array}\right]_{i}\left\{\begin{array}{l}
n_{x} \\
n_{y}
\end{array}\right\}_{i}|J|_{e} w_{s_{i}}
$$

Each element is transposed from the $x-y$ plane into a local plane of reference $r-s$. The expression $|J|_{a}$ in equation (2.39) refers to the determinant of the jacobian matrix of this transformation for an element $e$. The Gaussian quadrature rule is transposed from the standard $[-1,1]$ interval into the $r-s$ plane, according to the element side adjacent to the plot element and then transformed into the global $x-y$ coordinates:

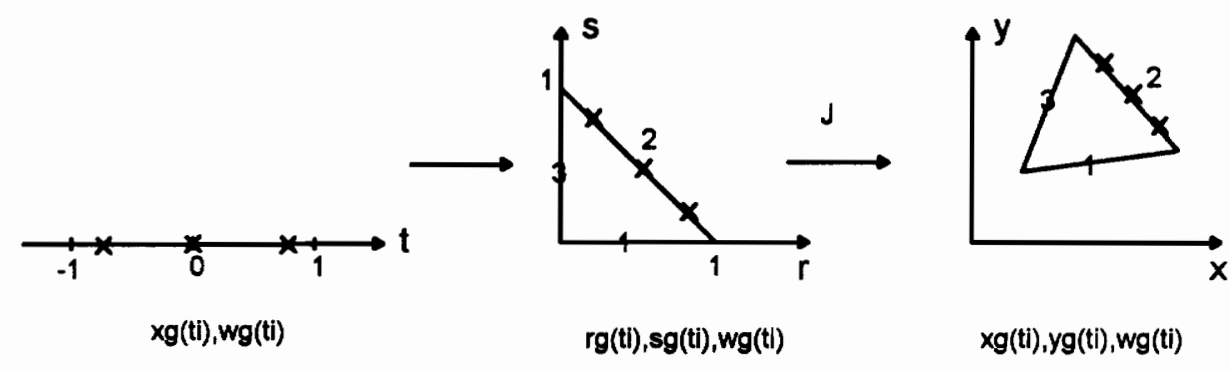

Figure 2.6 Geometrical transformation for Gauss rule (1-D)

To evaluate the tensor at gauss points, all its constitutive quantities must be defined at these same points. Since the velocity interpolation functions and their derivatives are defined in the local r-s plane, we must apply the chain rule to obtain the corresponding global values:

$$
\frac{d N^{u}}{d \mathbf{x}}\left(t_{i}\right)=\frac{d N^{u}}{d r} \frac{d r}{d \mathbf{x}}\left(t_{i}\right)+\frac{d N^{u}}{d s} \frac{d s}{d \mathbf{x}}\left(t_{i}\right)
$$

with

$$
\frac{d r, s}{d \mathbf{x}}\left(t_{i}\right)=\left[\frac{d \mathbf{x}}{d r, s}\right]^{-1}\left(t_{i}\right)=\left[\sum_{j=1}^{n n g} \frac{d N_{j}^{g}}{d r, s}\left(t_{i}\right) \mathbf{x}_{j}\right]^{-1}
$$


Similarly, the velocity at gauss points is found from:

$$
\mathbf{u}\left(t_{i}\right)=\sum_{j=1}^{n n u} N_{j}^{u}\left(t_{i}\right) \mathbf{u}_{j}
$$

Referring to (2.38) and using the two preceding results, we finally get the global velocity derivatives at gauss points. The pressure distribution over one element is defined linearly by:

$$
p(x)=p_{c}+\left[\frac{d p}{d x}\right]_{c}\left(x-x_{c}\right)+\left[\frac{d p}{d y}\right]_{c}\left(y-y_{c}\right)
$$

where $p_{c},[d p / d x]_{c}$, and $[d p / d y]_{c}$ are respectively the pressure and its gradient evaluated a the element centroid. Therefore, knowing the global gauss coordinates, (2.43) yields the pressure at the gauss points. We now possess every necessary component to compute the stress tensor at the gauss integration points. Once that result is achieved, the forces acting on the cylinder and the airfoil can finally be obtained from (2.39). 


\section{CHAPTER 3}

\section{Finite Element Method Applications}

This chapter presents a few applications of the finite element methodology discussed in chapter 2. First, we validate the computation of the aerodynamic forces acting on a surface by comparing the finite element approximations to some known solutions. Then we describe the problem of the flat plate with leading edge rotating cylinder in terms of a finite element problem.

\subsection{Calibration Examples}

\subsubsection{Boundary Layer (1-D)}

The first problem concerns a boundary-layer along a flat plate. The portion of the thin layer that is considered lays in the fully developed region so that no variation for the velocity exits along the $\mathbf{x}$ direction. The problem is then simplified to a one-dimensional case. It is an artificial problem that presents a rapid variation in one variable and is set to verify the finite-element methodology. From the fact that the velocity profile in a boundary-layer behaves asymptotically in the y direction, we can set the solution in the following form:

$$
\begin{aligned}
& u(y)=\left(1-e^{-R e y}\right) /\left(1-e^{-R e}\right), \\
& v=0 \\
& p(x)=x-0.2,
\end{aligned}
$$

for a domain described by figure 3.1. In order to obtain the solution stated by (3.1), we simulate body forces in the equations of motion. Instead of letting $f_{x}$ and $f_{y}$ be zero and solving for $u, v$ and $p$, we reverse the usual process by solving for the body forces. The Navier-Stokes equations (2.2) can be rewritten in the form:

$$
\begin{aligned}
& \rho\left(u \frac{\partial u}{\partial x}+v \frac{\partial u}{\partial y}\right)=-\frac{\partial P}{\partial x}+\mu\left(\frac{\partial^{2} u}{\partial x^{2}}+\frac{\partial^{2} u}{\partial y^{2}}\right)+f_{x} \\
& \rho\left(u \frac{\partial v}{\partial x}+v \frac{\partial v}{\partial y}\right)=-\frac{\partial P}{\partial y}+\mu\left(\frac{\partial^{2} v}{\partial x^{2}}+\frac{\partial^{2} v}{\partial y^{2}}\right)+f_{y}
\end{aligned}
$$


Replacing (3.1) into (3.2), we obtain for $f_{x}$ and $f_{y}$ :

$$
\begin{aligned}
& f_{x}(y)=1+(\operatorname{Re}) e^{-\operatorname{Re} y} /\left(1-e^{-\operatorname{Re}}\right) \\
& f_{y}=0
\end{aligned}
$$

These terms are given to the finite element solver. The finite element solution over the domain should therefore correspond approximately to (3.1).

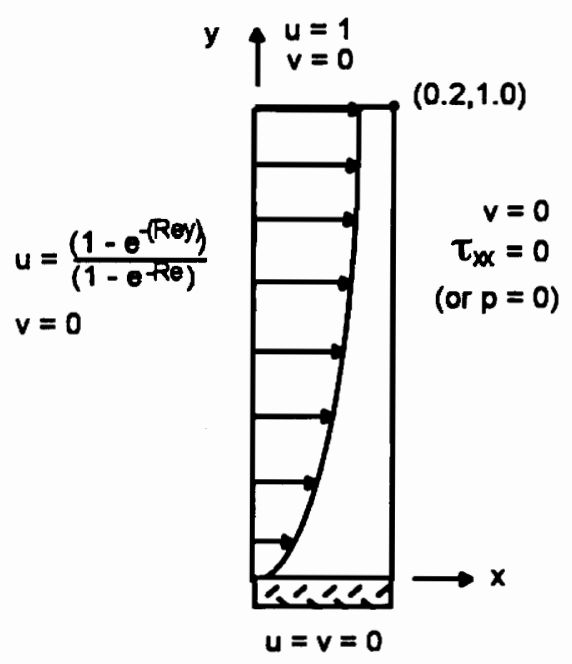

Figure 3.1 1-D BoundaryLayer Problem (1-D), Geometry

To compute the Cartesian forces acting on each side of the domain, we use equations (2.36) and (2.37):

$$
\mathbf{F}(x, y)=\int_{\Gamma}\left[\begin{array}{cc}
-p+2 \mu \frac{\partial u}{\partial x} & \mu\left(\frac{\partial u}{\partial y}+\frac{\partial v}{\partial x}\right) \\
\mu\left(\frac{\partial u}{\partial y}+\frac{\partial v}{\partial x}\right) & -p+2 \frac{\partial v}{\partial y}
\end{array}\right]_{\Gamma}\left\{\begin{array}{l}
n_{x} \\
n_{y}
\end{array}\right\}_{\Gamma} d \Gamma
$$


Setting values for $x$ and $y$ in (3.4), we get an expression the force vector on each boundary:

$$
\begin{aligned}
& F_{\text {left }}=\int_{0}^{1}\left[\begin{array}{cc}
-x+0.2 & e^{-R e y} /\left(1-e^{-R e}\right) \\
e^{-R e y} /\left(1-e^{-R o}\right) & -x+0.2
\end{array}\right]\left\{\begin{array}{l}
1 \\
0
\end{array}\right\} d y=\left\{\begin{array}{c}
0.2 \\
1 / R e
\end{array}\right\} \\
& F_{\text {right }}=\int_{0}^{1}\left[\begin{array}{cc}
0 & e^{-R e y} /\left(1-e^{-R o}\right) \\
e^{-R e y} /\left(1-e^{-R e}\right) & 0
\end{array}\right]\left\{\begin{array}{c}
-1 \\
0
\end{array}\right\} d y=\left\{\begin{array}{c}
0 \\
-1 / R e
\end{array}\right\} \\
& F_{\text {top }}=\int_{0}^{02}\left[\begin{array}{cc}
-x+0.2 & e^{-R_{\theta}} /\left(1-e^{-R_{\theta}}\right) \\
e^{-R_{0}} /\left(1-e^{-R_{0}}\right) & -x+0.2
\end{array}\right]\left\{\begin{array}{c}
0 \\
-1
\end{array}\right\} d x=\left\{\begin{array}{c}
-0.2 e^{-R_{\theta}} /\left(1-e^{-R_{\theta}}\right) \\
-0.02
\end{array}\right\} \\
& F_{\text {bottom }}=\int_{0}^{0.2}\left[\begin{array}{cc}
-x+0.2 & 1 /\left(1-e^{-R e}\right) \\
1 /\left(1-e^{-R e}\right) & -x+0.2
\end{array}\right]\left\{\begin{array}{l}
0 \\
1
\end{array}\right\} d x=\left\{\begin{array}{c}
0.2 /\left(1-e^{-R e}\right) \\
0.02
\end{array}\right\}
\end{aligned}
$$

Following the scheme shown in figure 2.1, the first step to the finite element method is to provide the equations solver with the problem definition and a coarse hand-made grid. A uniform grid of two elements with a uniform size function was used as the first backmesh (see figure 3.2).

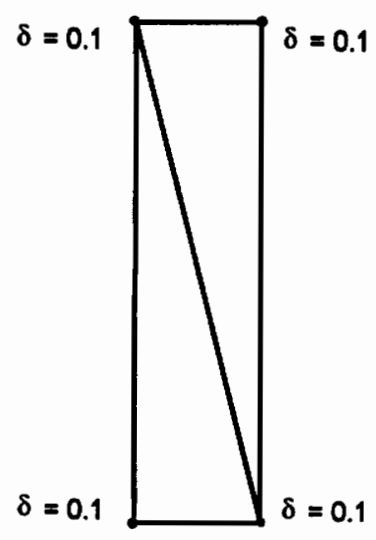

Figure 3.2 Boundary Layer Problem (1-D), First Backmesh

The grid adaptation sequence is illustrated in figure 3.3. The mesh (a) has a uniform distribution of elements $(\delta=0.1)$ and is constructed with the coarse grid of figure 3.2. 

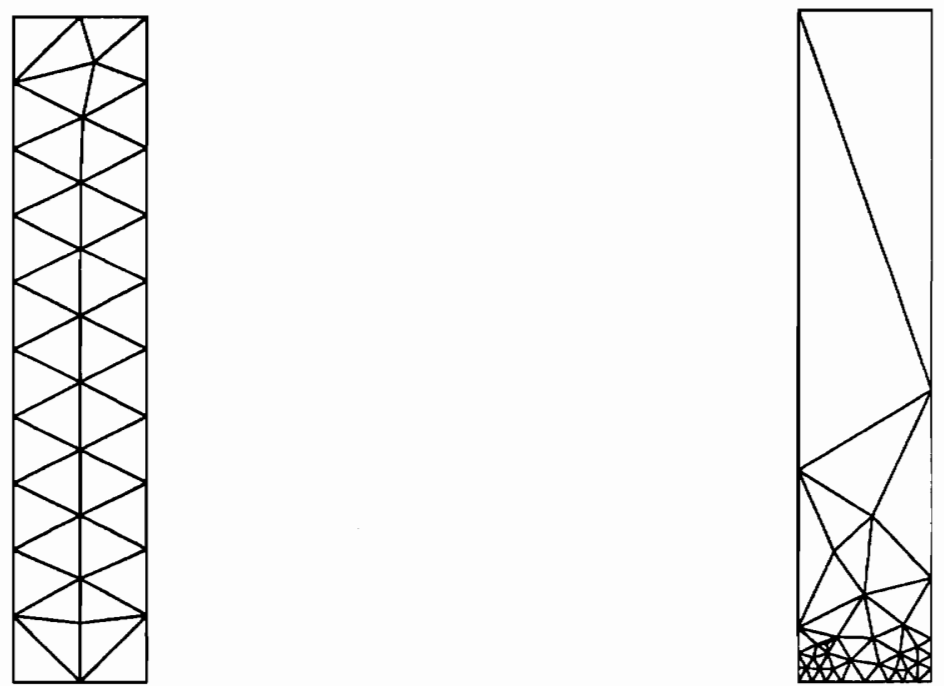

(c)

(d)
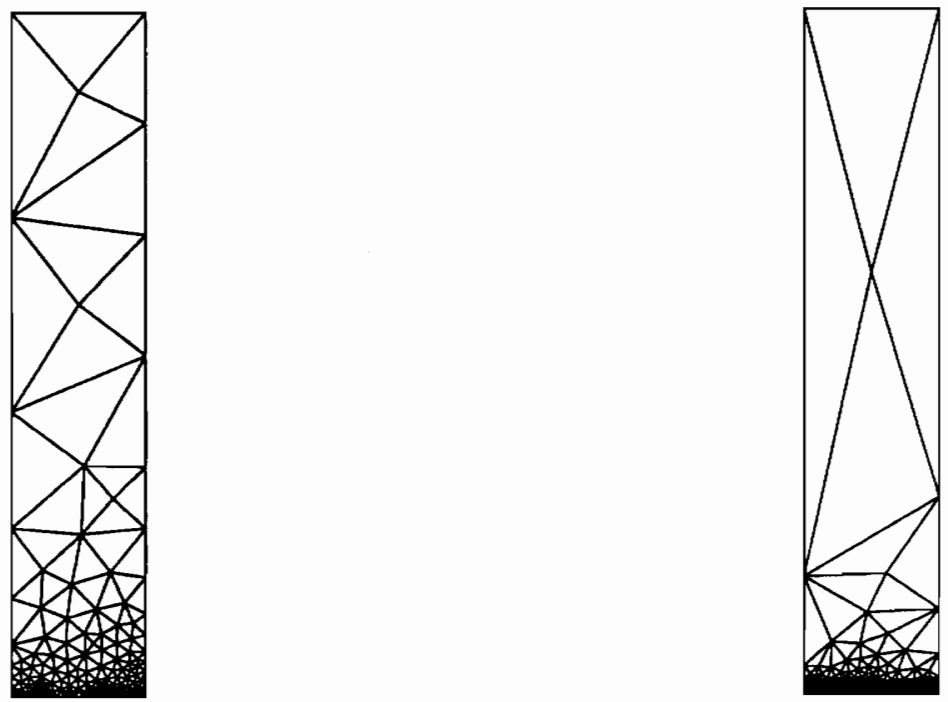

Figure 3.3 Boundary-Layer Problem (1-D), Mesh Convergence

After we have obtained the first finite element solution, we analyse the error distribution over the domain. Almost always, the error on the first grid (Figure 3.3 (a)) is non negligible so we must generate a new grid that will better capture the problem characteristics. Since we know the analytical solution to this problem, we use the exact error to compute the size function for the next grid. 
The selection of the transition operator (cf. section 1.4) is arbitrary. Most often, we choose to reduce the error by a certain percentage. In general, both operators don't reach the desired target in one cycle, particularly if the actual relative error is too large. This phenomenon is explained by the adaptative strategy based on (2.30). This rule comes from the interpolation theory and is exact only in an asymptotic way. Consequently, the error must already be small for (2.30) to be verified. In short, either one or the other gives good results after a few cycles, the choice relies on the user's judgment and experience. Because the complexity of the problem lies in the velocity field, we exclude the pressure from the error evaluation by using the energy norm instead of the Stokes norm. The finite-element approximation representing the velocity field quadratically, the error will be more important for the velocity which behaves asymptotically than for the pressure. We see in figure 3.4 the tendency of the adaptive module to produce an important concentration of elements in the region of the boundary layer, where the velocity gradients are high. In figure 3.7 a), the error is spread in the lower region of the domain. For this reason, many small elements are placed in this area while large ones covers the rest of the domain were the velocity field is uniform (see figure 3.3 (b), (c) and (d)). In the end, the largest error, however still quite small $(\mathrm{O}(\mathrm{e}-05))$, lies in the very bottom of the computational region (figure $3.7 \mathrm{~b}$ )). The results of the mesh adaptivity strategy is reviewed in table 3.1.

Table 3.1 Boundary-Layer problem, Adaptivity Strategy

\begin{tabular}{|c|c|c|c|c|c|}
\hline Cycle & $\begin{array}{c}\text { Absolute } \\
\text { Error } \\
\text { Energy } \\
\text { norm }\end{array}$ & $\begin{array}{c}\text { Solution } \\
\text { Energy } \\
\text { norm }\end{array}$ & $\begin{array}{c}\text { Relative } \\
\text { Error }\end{array}$ & $\begin{array}{c}\text { Transition } \\
\text { Operator }\end{array}$ & $\begin{array}{c}\text { Target Rel. } \\
\text { Error }\end{array}$ \\
\hline \hline (a) & $1.360 \mathrm{e}-02$ & $2.968 \mathrm{e}-03$ & 4.6 & reduction & 0.10 \\
\hline (b) & $1.660 \mathrm{e}-02$ & $7.648 \mathrm{e}-03$ & 2.2 & reduction & 0.10 \\
\hline (c) & $6.667 \mathrm{e}-03$ & $1.260 \mathrm{e}-02$ & 0.53 & fixe & 0.025 \\
\hline (d) & $6.321 \mathrm{e}-04$ & $1.413 \mathrm{e}-02$ & $4.47 \mathrm{e}-02$ & & \\
\hline
\end{tabular}




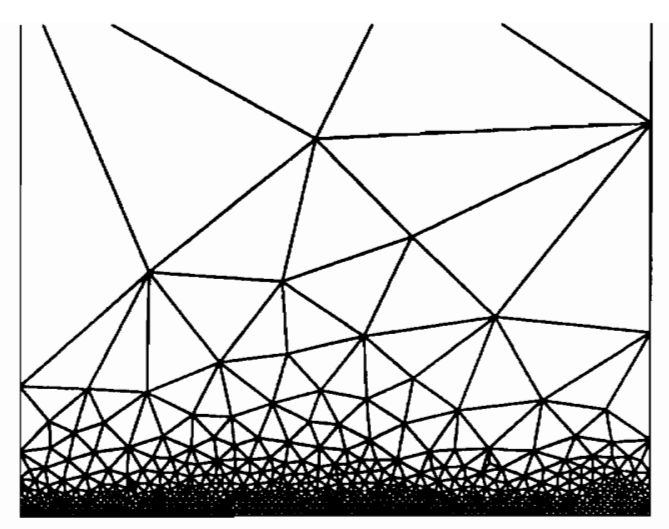

Figure 3.4 Boundary-layer problem (1-D), Mesh Detail of fig. 3.3 (d)

Figures 3.5 and 3.6 show, respectively, the velocity and pressure distributions of the final finite element solution. The velocity profile is typical of a boundary-layer where the vertical component is zero and the horizontal component quickly rises from zero to the free stream velocity within the boundary-layer width (approximately 0.0048 , see figure 3.5). In figure 3.6, we see that the pressure behaves as described in (3.1); it is constant in the $\mathrm{y}$ direction and varies in the $\mathrm{x}$ direction from -0.2 to 0 within the domain. Table 3.2 shows a comparison between the aerodynamic forces computed from the finite element solution and the analytical solution for a Reynold number of 1000 .

Table 3.2 Comparison between analytical results and f.e.m. solution for the one-dimensional boundarylayer problem.

\begin{tabular}{|c||c|c|c|c|c|}
\cline { 2 - 6 } \multicolumn{1}{c||}{} & \multicolumn{2}{c|}{ Analytical Solution } & \multicolumn{2}{c|}{ Finite Element Method } & $\begin{array}{c}\text { Relative } \\
\text { Error }\end{array}$ \\
\hline Boundary & $\mathrm{F}_{\mathrm{x}}$ & $\mathrm{F}_{\mathrm{v}}$ & $\mathrm{F}_{\mathrm{x}}$ & $\mathrm{F}_{\mathrm{v}}$ & $\mathrm{e}_{\mathrm{F} \mid}$ \\
\hline \hline Left & 0.2 & 0.001 & 0.200053 & $8.97181 \mathrm{e}-04$ & $2.626 \mathrm{e}-04$ \\
\hline Right & 0 & -0.001 & $2.20129 \mathrm{e}-05$ & $-9.85138 \mathrm{e}-04$ & $1.462 \mathrm{e}-02$ \\
\hline Top & 0 & -0.02 & $-7.88065 \mathrm{e}-10$ & -0.0200008 & $4.000 \mathrm{e}-04$ \\
\hline Bottom & 0.2 & 0.02 & 0.187451 & 0.0200531 & $6.208 \mathrm{e}-02$ \\
\hline
\end{tabular}




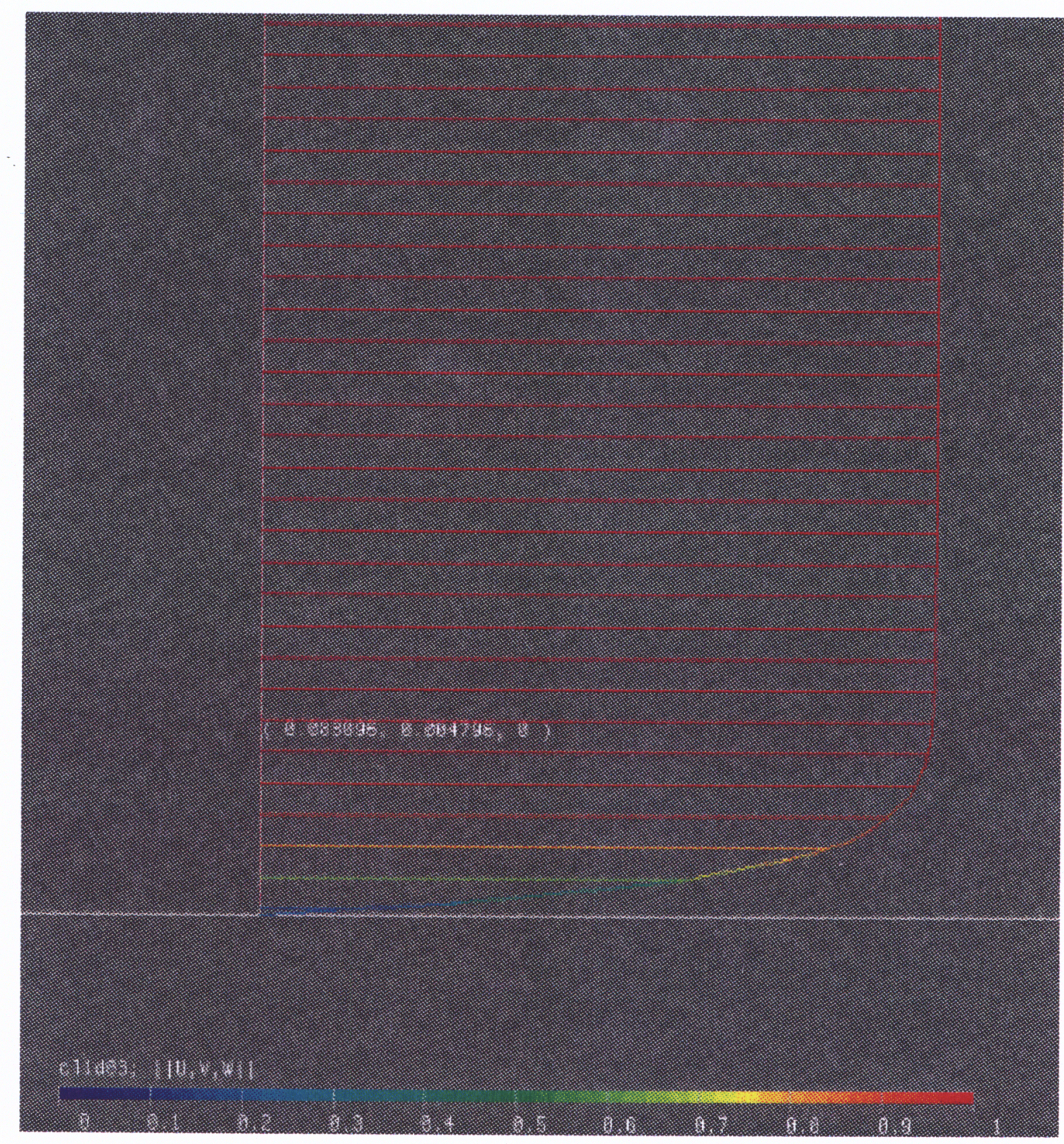

Figure 3.5 Boundary-layer Problem (1-D), velocity Profile 


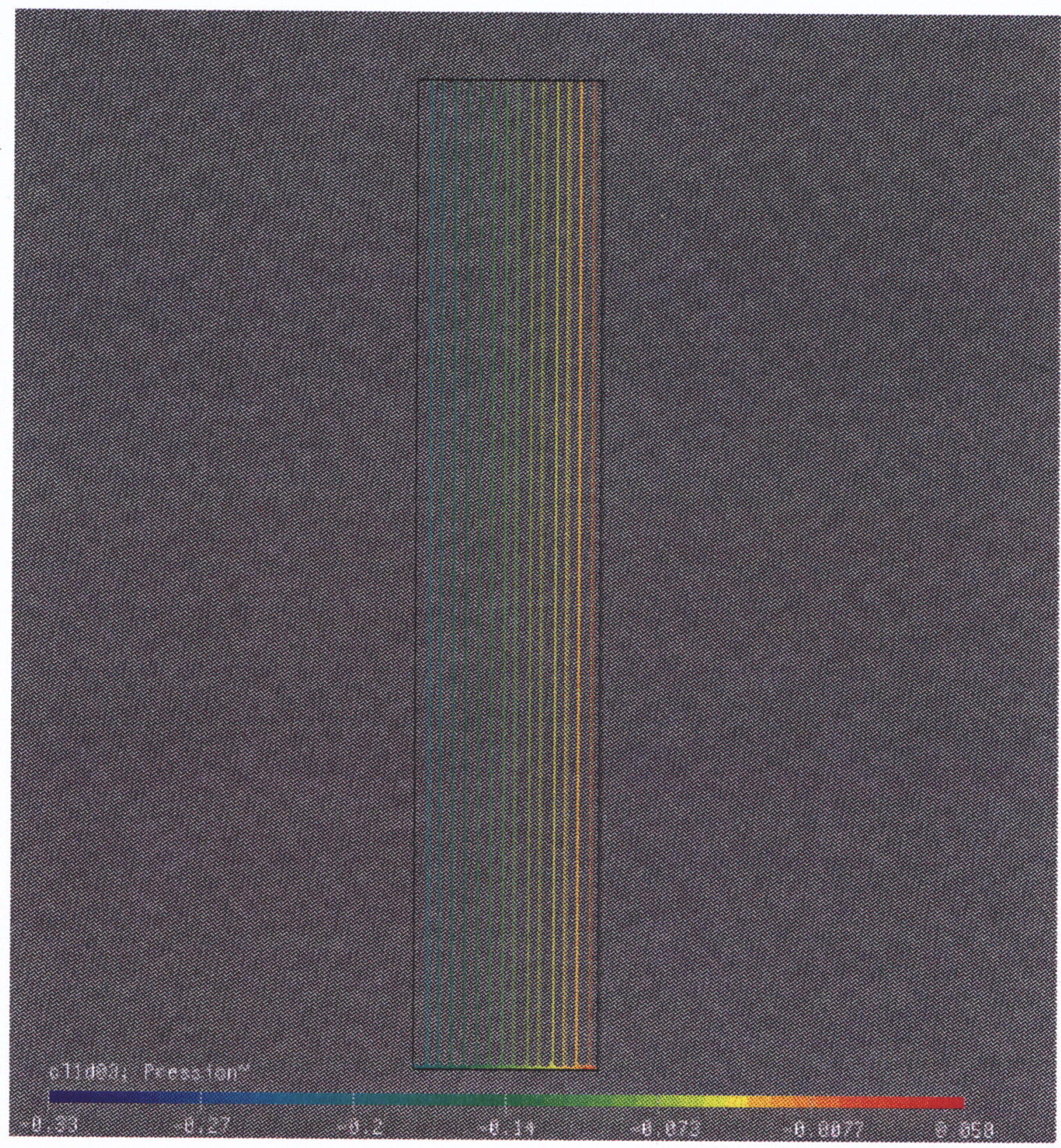

Figure 3.6 Boundary-Layer Problem (1-D), Pressure Distribution 
a) Cycle (a) in Figure 3.3

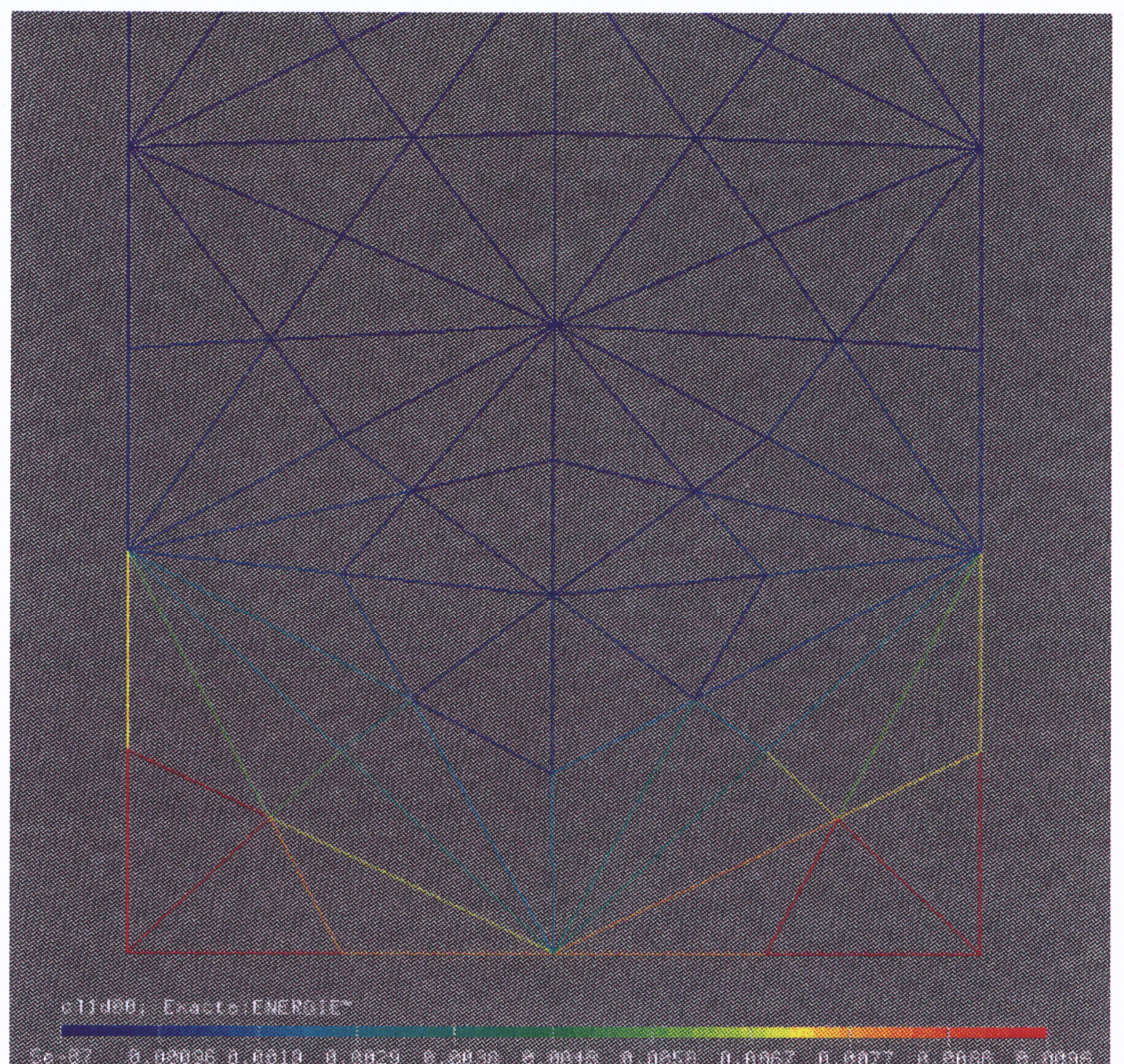

Figure 3.7 Boundary-Layer Problem (1-D), Error Distribution 
b) Cycle (d) in Figure 3.3

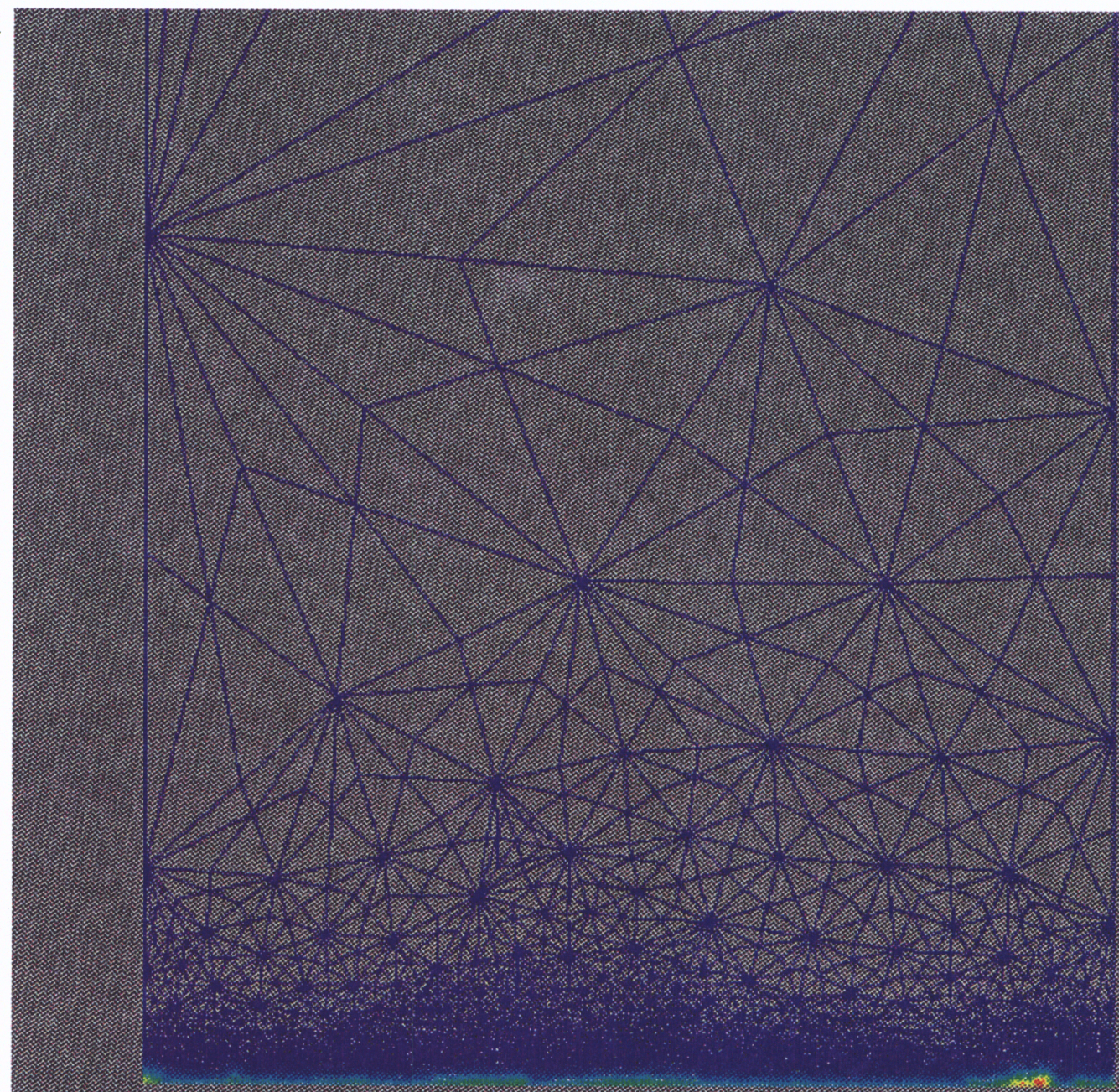

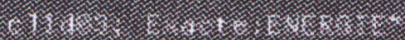

1.

Figure 3.7 Boundary-Layer Problem (1-D), Error Distribution 
The largest error in the results summarized by table 3.2 lies in the bottom boundary since it is where the flow is most complex, thus generating more error. Let's mention that, in general, the error on the derivatives of a function is larger than the error on the function itself. Therefore, there will be more error on the computed aerodynamic forces than on the velocity distribution because the stress tensor involves the derivatives of the velocity field.

From Table 3.2, we see that the finite element modeling provides a reliable approximation to the solution of the Navier-Stokes equations. However, this problem is quite a simple flow situation and further investigations should be made in order to assure the validity of the methodology. 


\subsubsection{Two-Dimensional Half-Plates}

To study a more realistic representation of the flow about a flat plate, we model three plates of finite length and non negligible thickness. The analysis is based on J. Caillé and J.A. Schetz numerical study of a flow about a flat plate2r. The computer models are shown in figure 3.8.

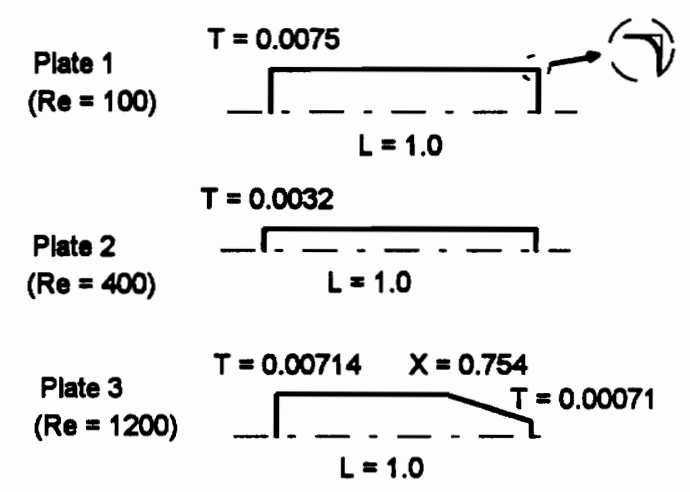

Figure 3.8 Two-Dimensional Half Plates, Geometry

Figure 3.9 indicates the computational region and its boundary conditions. Caillé \& Schetz based the upstream boundary location $X_{\text {up }}$ on Walter and Larsen ${ }^{27}$ results and fixed the top boundary condition $Y_{\text {top }}$ to twice the boundary-layer thickness predicted by the Blasius solution.

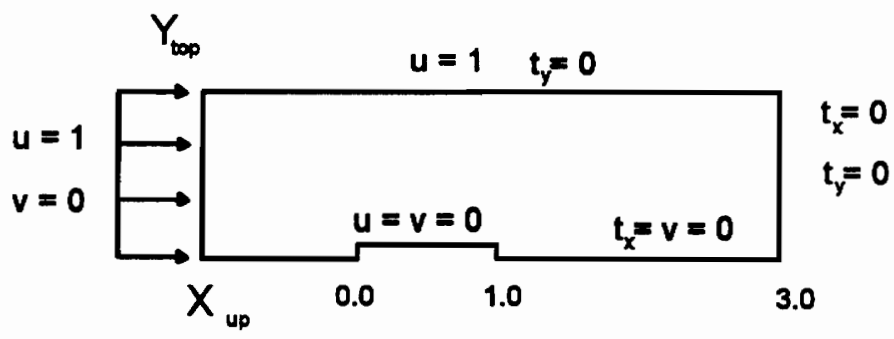

Figure 3.9 Two-Dimensional Half-Plates, Computational region and boundary conditions

Table 3.3 Characteristics of the domain for the two-dimensional half-plates

\begin{tabular}{|c|c|c|}
\hline $\operatorname{Re}$ & $\mathrm{X}_{\text {up }}$ & $\mathrm{Y}_{\text {top }}$ \\
\hline \hline 100 & -2.00 & 1.000 \\
\hline 400 & -1.00 & 0.500 \\
\hline 1200 & -0.50 & 0.400 \\
\hline
\end{tabular}


To keep the mesh in reasonable proportions, we soften the singularities created from the half-plate sharp corners by replacing them with small quarter circles. For each half-plate, the mesh is refined twice using the reduction transition operator (target of 0.25 the first time and a target of 0.20 the second time). Since we don't know the exact solution to this problem, we use the error estimator described in section 2.3. The relative error over each final mesh is around one percent. Figure 3.11 shows the mesh adaptivity sequence for the half-plate with $\mathrm{Re}=1200$. Again, we notice a tendency to concentrate many small elements in the regions of high pressure and velocity gradients, near the corners of the plate. In figure $3.14 \mathrm{a}$ ), we observe more error near the left corner of the plate. This is taken into account by the adaptive module which places many small elements around that area in the next grid (figure 3.11 (b)). In the next cycle, error in the wake of the plate (figure $3.14 \mathrm{~b}$ )) produces a larger concentration of elements behind the plate in figure 3.11 (c).

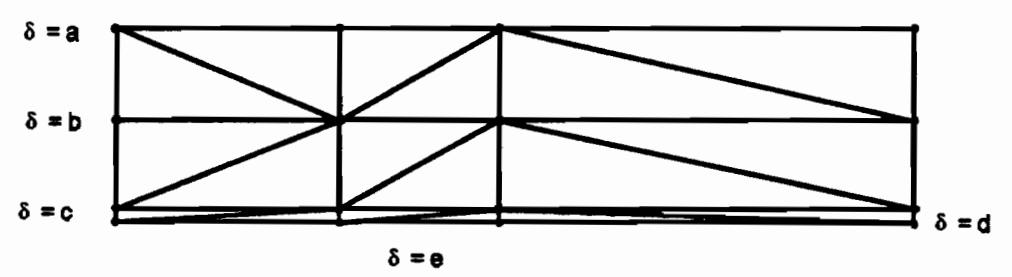

Figure 3.10 Two-Dimensional Half-Plates, First Backmesh

Table 3.4 Two-Dimensional Half-Plates, size function for the initial backmesh

\begin{tabular}{|c|c|c|c|c|c|}
\hline Plate & $\mathrm{a}$ & $\mathrm{b}$ & $\mathrm{c}$ & $\mathrm{d}$ & $\mathrm{e}$ \\
\hline \hline 1 & 1.0 & 0.5 & 0.75 & 0.15 & 0.0375 \\
\hline 2 & 0.5 & 0.25 & 0.32 & 0.064 & 0.016 \\
\hline 3 & 0.4 & 0.2 & 0.071 & 0.0284 & 0.0284 \\
\hline
\end{tabular}

The initial backmesh is based on the same pattern for the three plates. In figure 3.10, the function size has the same value $\delta=\mathrm{a}$ for the four top nodes, $\delta=\mathrm{b}$ for the four middle nodes, $\delta=\mathrm{c}$ for the two upstream nodes, $\delta=\mathrm{d}$ for the two downstream nodes and $\delta=\mathrm{e}$ for the plate nodes. Table 3.3 lists the $\mathrm{a}, \mathrm{b}, \mathrm{c}, \mathrm{d}$ and e values for each plate. 
(a)

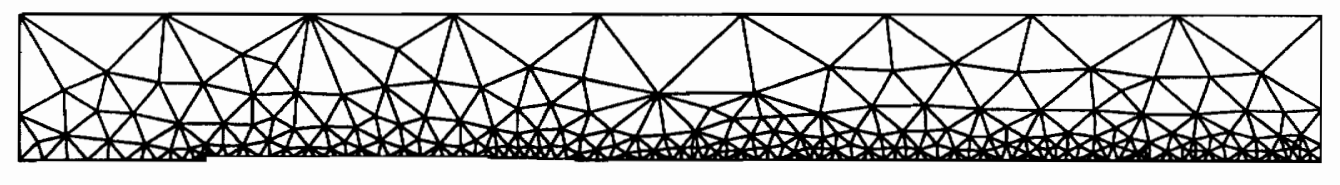

(b)

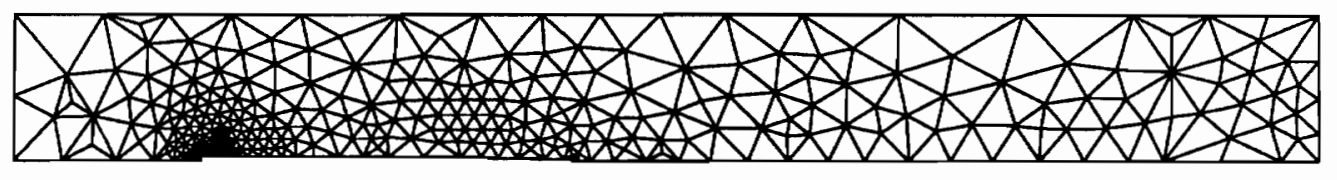

(c)

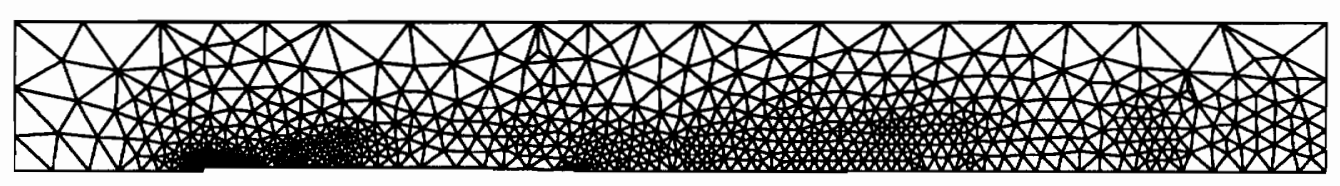

Figure 3.11 Two-Dimensional Half-Plates, Mesh Convergence (case Re $=1200$ )

Figures 3.12 and 3.13 present velocity and pressure distribution on the final mesh for the half-plate at $\operatorname{Re}=1200$. Both figures presents high gradients in the vicinity of the plate's corners which are responsible for the need of a fine discretization in that area. 


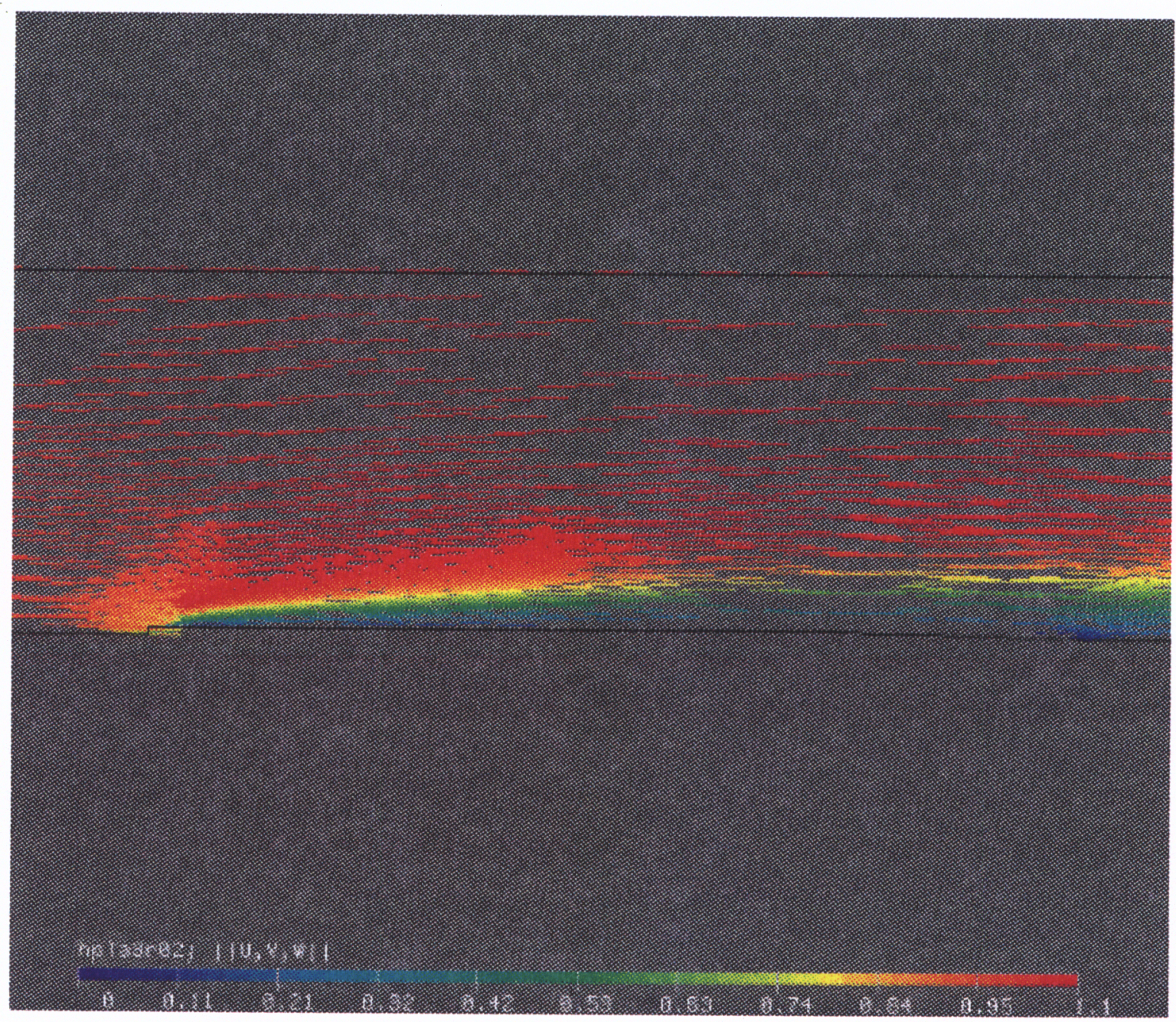

Figure 3.12 Two-Dimensional Half-Plate, $\operatorname{Re}=1200$, Velocity Distribution 


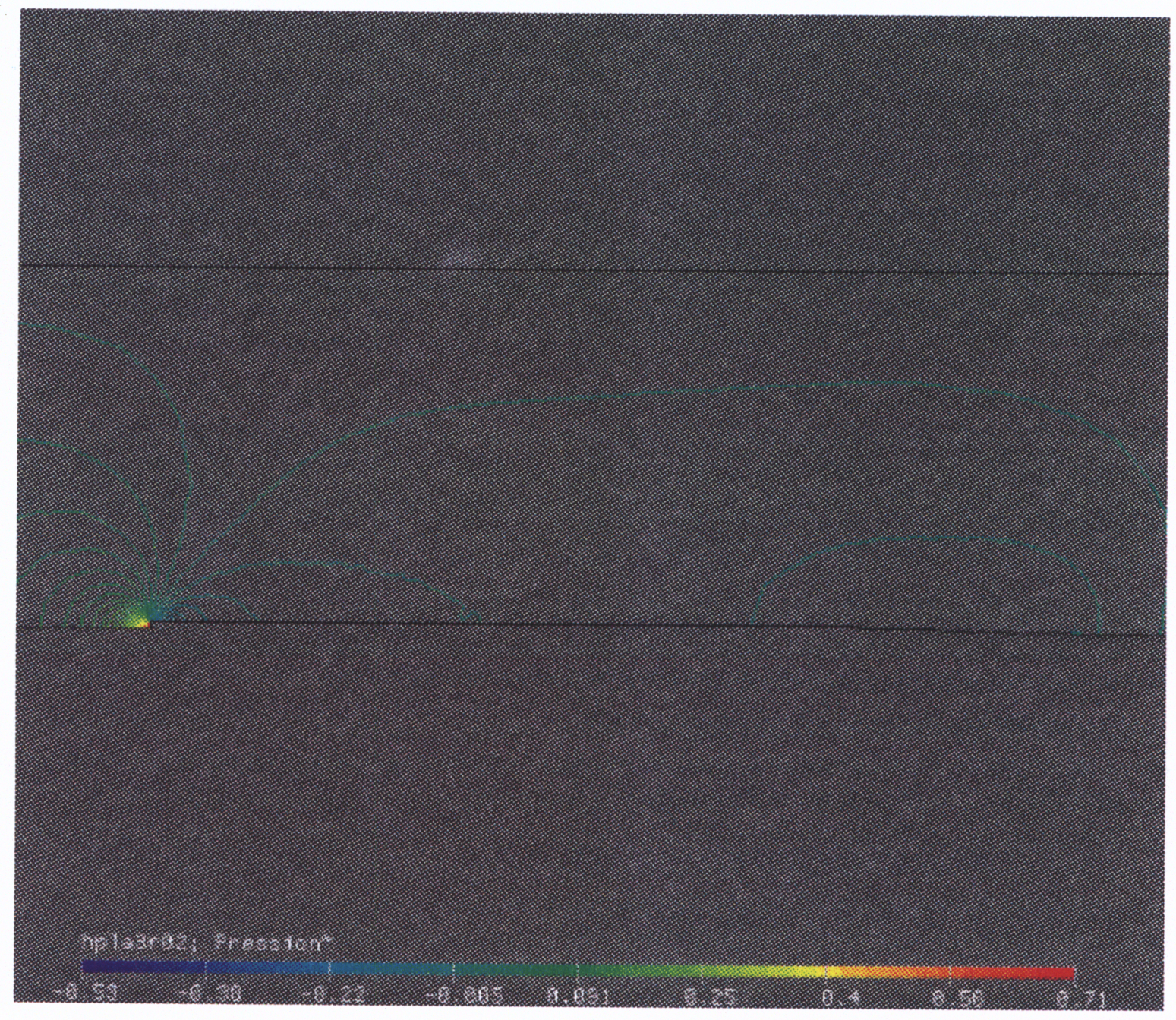

Figure 3.13 Two-Dimensional Half-Plate, Re $=1200$, Pressure Distribution 
a) Cycle (a) in Figure 3.11

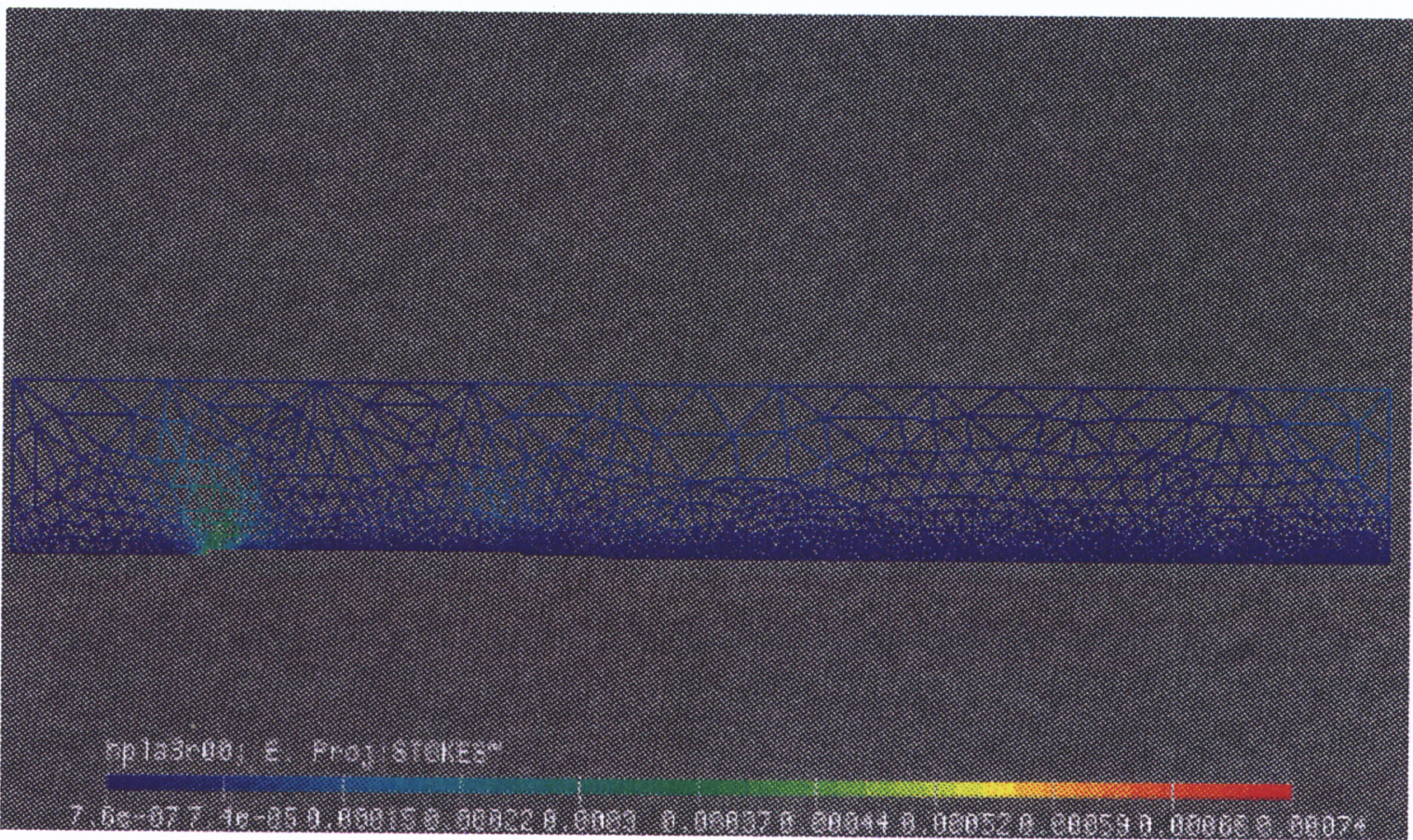

Figure 3.14 Two-Dimensional Half-Plate, $\mathrm{Re}=1200$, Error Distribution 
b) Cycle (b) in Figure 3.11

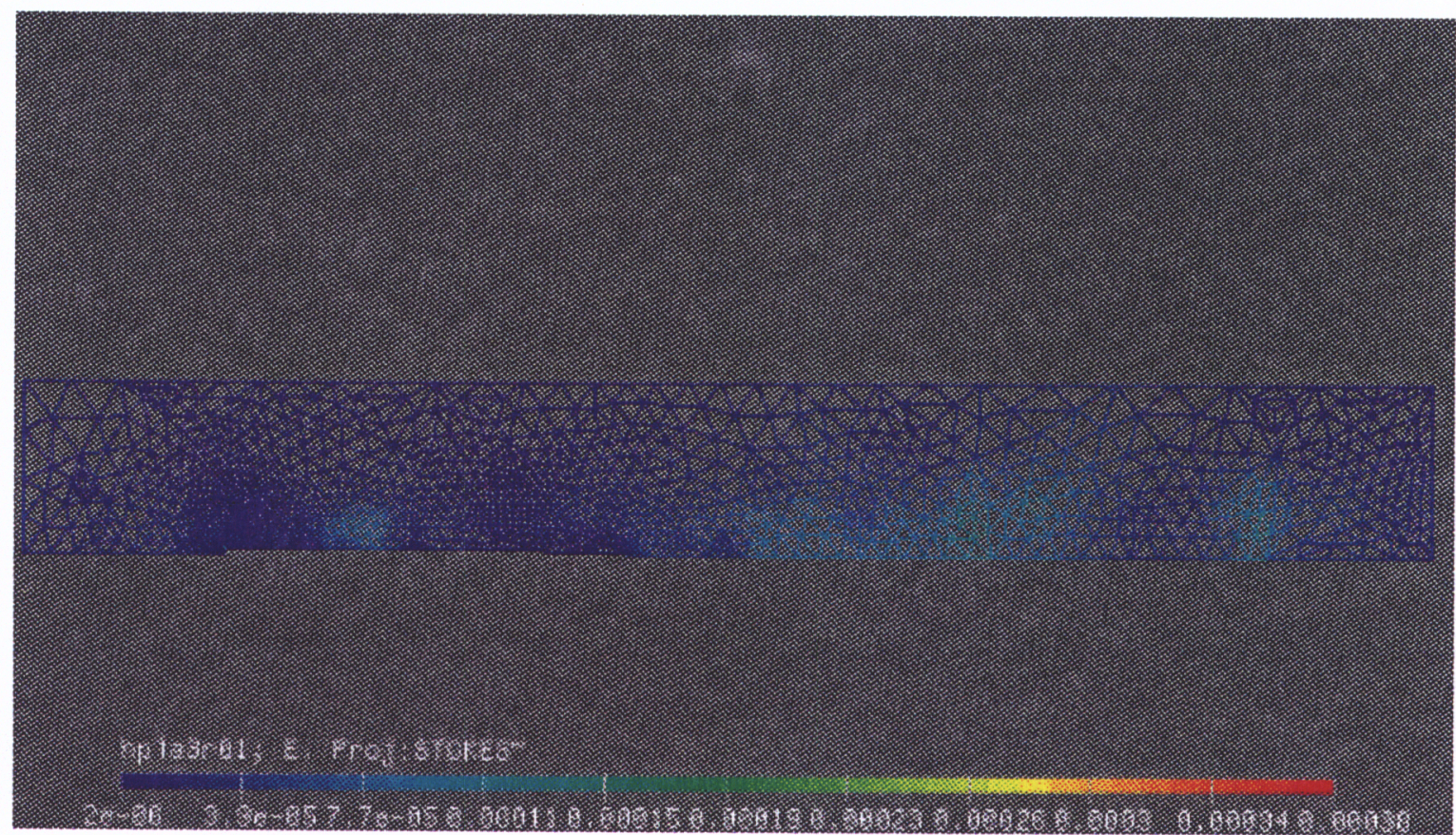

Figure 3.14 Two-Dimensional Half-Plate, $\mathrm{Re}=1200$, Error Distribution 
Caille and Schetz studied the skin friction coefficient over each half-plate as well as the wake centerline velocity. From the finite element solutions, we computed the forces acting on the half-plates surface and deduced the skin friction coefficient. The skin friction coefficient is defined as the tangential force acting on the surface divided by the dynamic pressure $\left(\frac{1}{2} \rho U_{\infty}^{2} L\right)$. Here, the dynamic pressure is equal to $(1 / 2)$ since the density, the free stream velocity and the characteristic lentgh are equal to unity. Therefore, the skin friction coefficient is twice the tangential force.

Since the finite element method is implemented in the Cartesian coordinate system, we must transform the resulting Cartesian forces in the normal-tangential system. This is easily accomplished with a simple rotation transformation about the $\mathrm{z}$ axis:

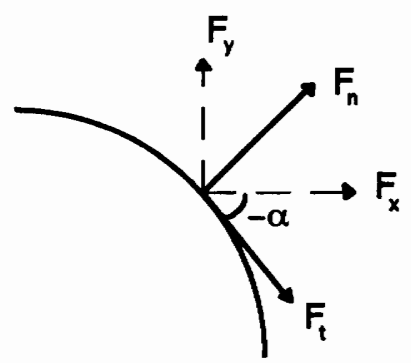

Figure 3.15 Transformation from Cartesian to Normal-Tangential system

$$
\left\{\begin{array}{l}
F_{t} \\
F_{n}
\end{array}\right\}=\left[\begin{array}{cc}
\cos (-\alpha) & \sin (-\alpha) \\
-\sin (-\alpha) & \cos (-\alpha)
\end{array}\right]\left\{\begin{array}{l}
F_{x} \\
F_{y}
\end{array}\right\}
$$

Figures 3.16 through 3.18 compare the curves obtained from the finite-element solution with Caillé \& Schetz ${ }^{26}$ results. 
a) Skin-Friction Coefficient Distribution over the plate

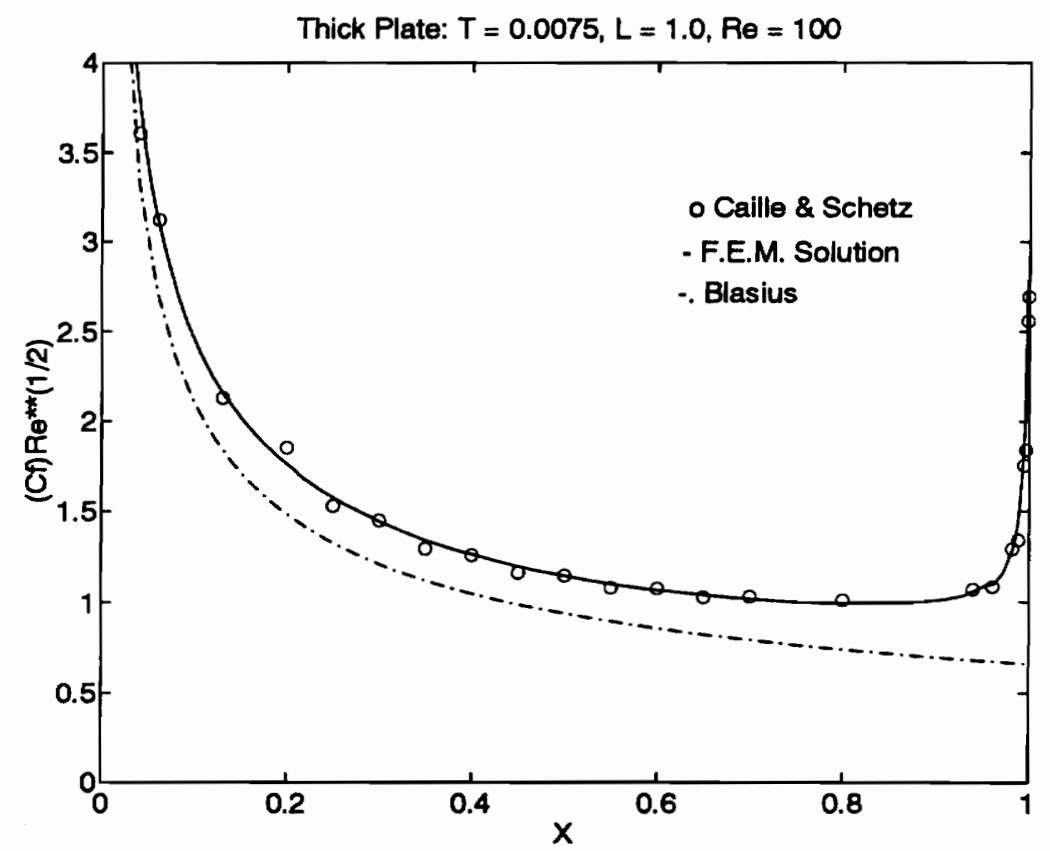

b) Wake Centerline Velocity

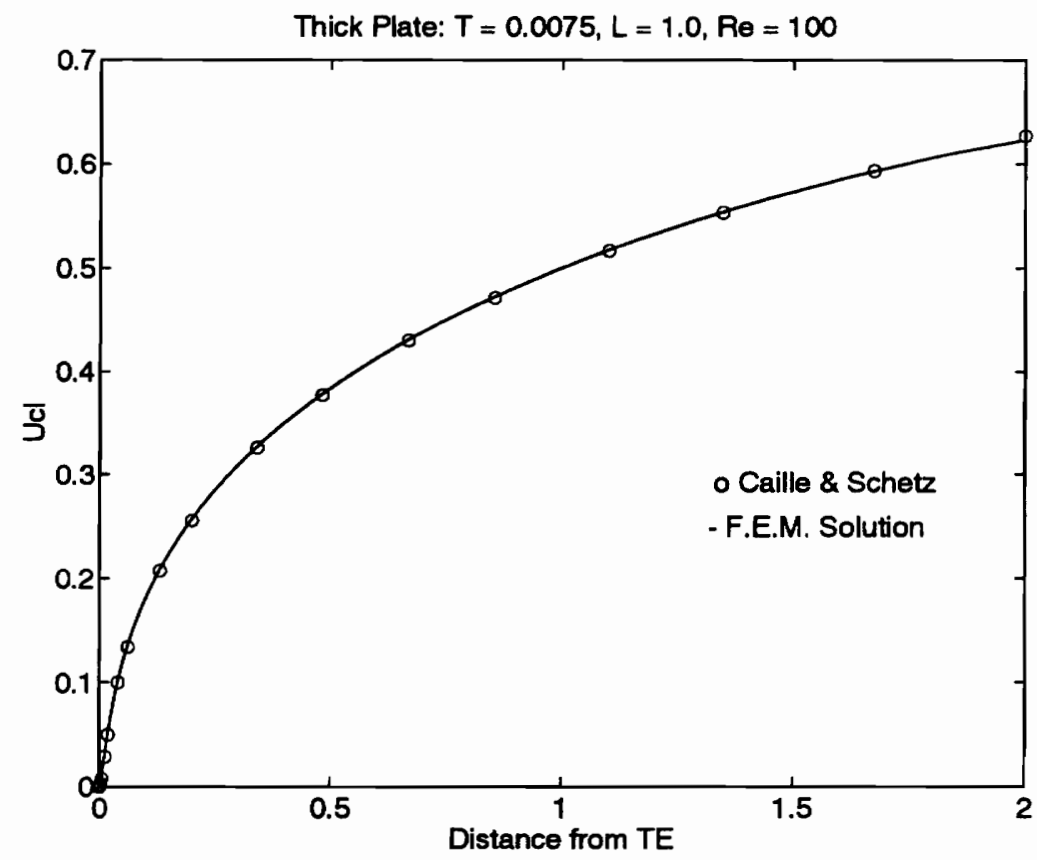

Figure 3.16 Two-dimensional Half-Plate, $\mathrm{Re}=100$ 
a) Skin-Friction Coefficient Distribution over the plate

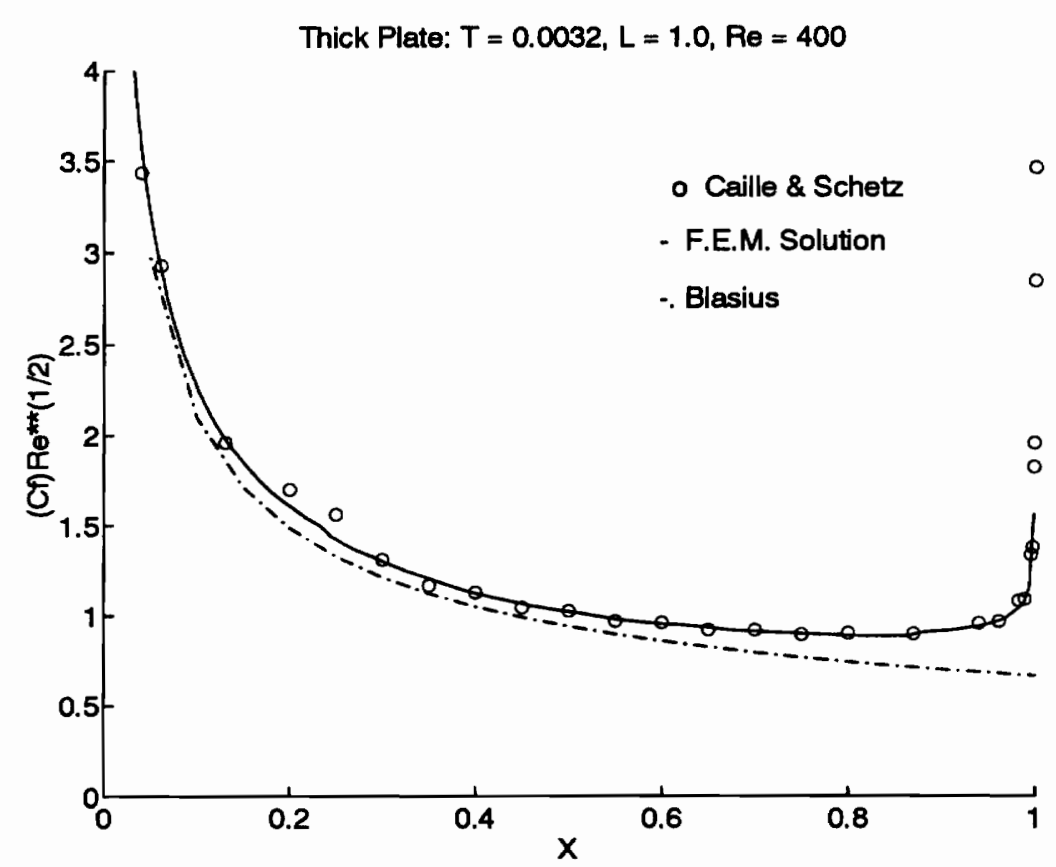

b) Wake Centerline Velocity

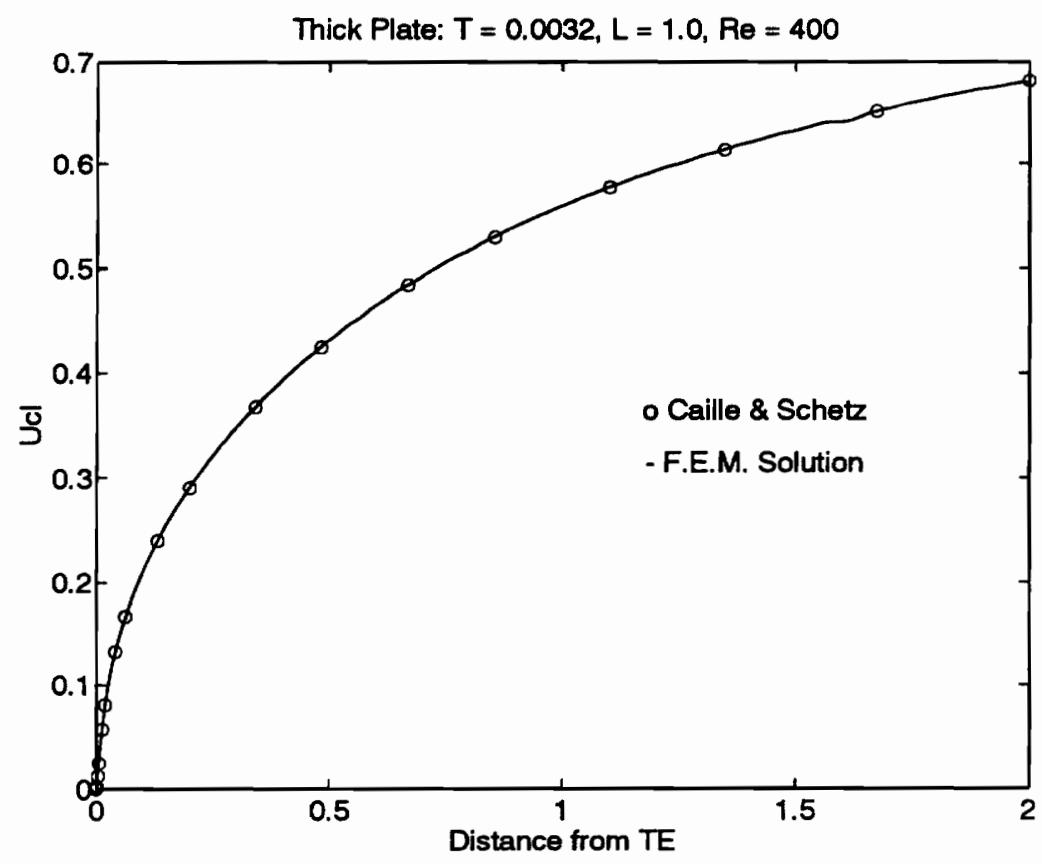

Figure 3.17 Two-dimensional Half-Plate, $\mathrm{Re}=400$ 
a) Skin-Friction Coefficient Distribution over the plate

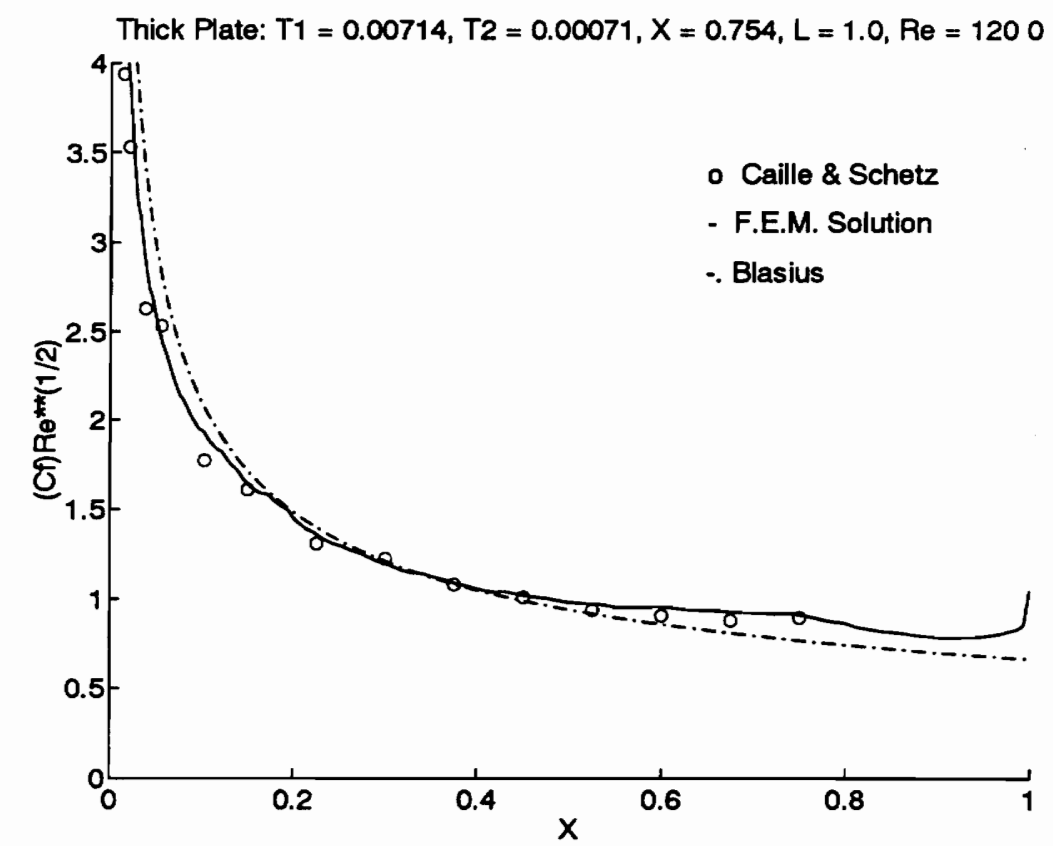

b) Wake Centerline Velocity

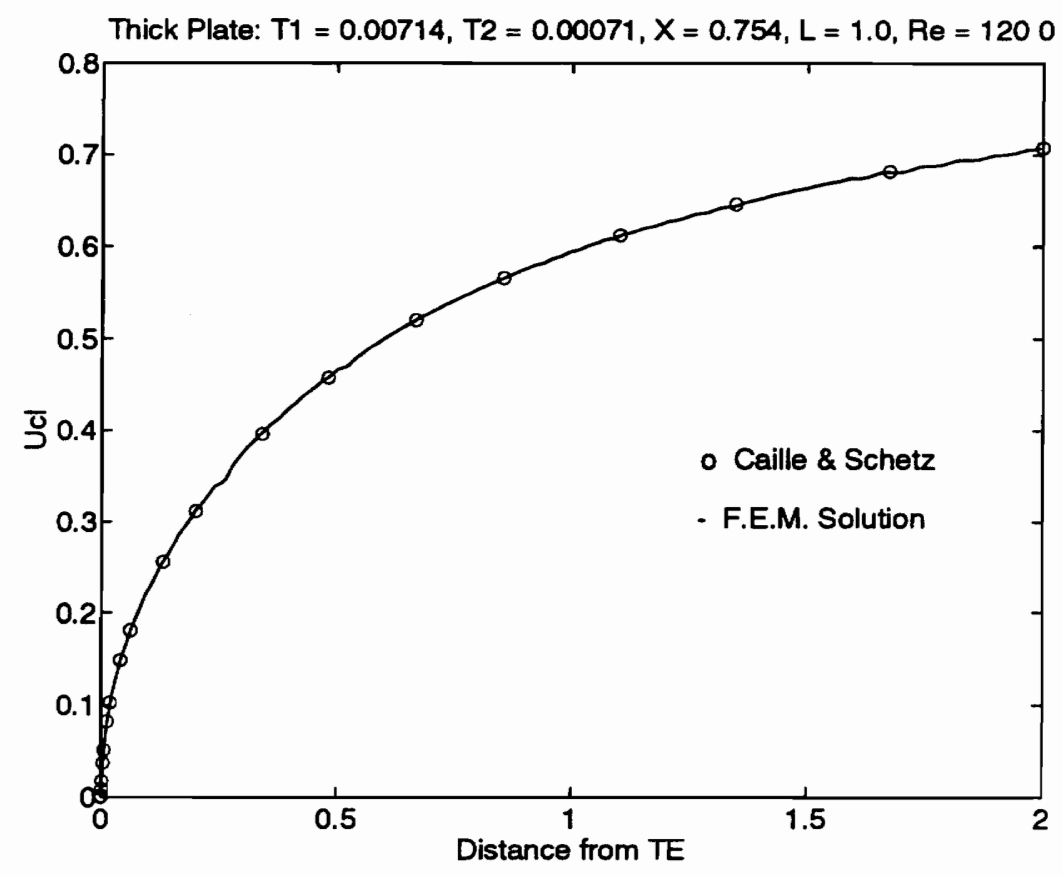

Figure 3.18 Two-dimensional Half-Plate, $\operatorname{Re}=1200$ 
We see from these results that the finite-element solution for the flow behavior about a flat plate responds well to a more elaborate geometry. However, in the present study, a rotating cylinder increases the complexity of the flow. In order to understand the flow behavior about a rotating cylinder, we next solve the problem of two concentric cylinders rotating at different speeds. 


\subsubsection{Two Concentric Rotating Cylinders}

The problem of two cylinders rotating at different speeds about the same axis can be solved analytically from the Navier-Stokes equations (in cylindrical form):

$$
\begin{aligned}
& \rho\left(\frac{D v_{r}}{D t}-\frac{v_{\theta}}{r}\right)=-\frac{\partial P}{\partial r}+\mu\left(\nabla^{2} v_{r}-\frac{v_{r}}{r^{2}}-\frac{2}{r^{2}} \frac{\partial v_{\theta}}{\partial \theta}\right) \\
& \rho\left(\frac{D v_{\theta}}{D t}+\frac{v_{r} v_{\theta}}{r}\right)=-\frac{1}{r} \frac{\partial P}{\partial \theta}+\mu\left(\nabla^{2} v_{\theta}+\frac{2}{r^{2}} \frac{\partial v_{r}}{\partial \theta}-\frac{v_{\theta}}{r^{2}}\right)
\end{aligned}
$$

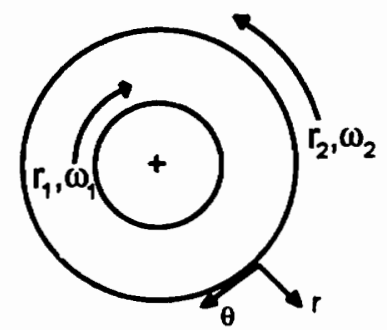

Figure 3.19 Concentric Rotating Cylinders Problem, Geometry

Assuming steady-flow conditions and radial symmetry, we find that:

$$
\begin{aligned}
& v_{r}=0, v_{\theta}=f(r) \\
& p=f(r)
\end{aligned}
$$

Replacing (3.11) in (3.10), we get

$$
\begin{aligned}
& \mu\left(\frac{d^{2} v_{\theta}}{d r^{2}}+\frac{1}{r} \frac{d v_{\theta}}{d r}-\frac{v_{\theta}}{r^{2}}\right)=0 \\
& \frac{d p}{d r}=\frac{\rho v_{\theta}^{2}}{r}
\end{aligned}
$$

with the boundary-conditions:

$$
\begin{aligned}
& v_{\theta}\left(r_{1}\right)=r_{1} \omega_{1} \\
& v_{\theta}\left(r_{2}\right)=r_{2} \omega_{2}
\end{aligned}
$$


(3.12) and (3.13) can be solved for $p(r)$ and $v_{\theta}(r)$ :

$$
\begin{aligned}
& v_{\theta}(r)=\alpha r+\frac{\beta}{r} \\
& p(r)=\rho\left(\frac{\alpha^{2} r^{2}}{2}-\frac{\beta^{2}}{2 r^{2}}+2 \alpha \beta \ln (r)\right)+\text { const }
\end{aligned}
$$

with

$$
\begin{aligned}
& \alpha=\frac{\omega_{1} r_{1} / r_{2}-\omega_{2} r_{2} / r_{1}}{r_{1} / r_{2}-r_{2} / r_{1}} \\
& \beta=\frac{\left(\omega_{2}-\omega_{1}\right) r_{1} r_{2}}{r_{1} / r_{2}-r_{2} / r_{1}} .
\end{aligned}
$$

To compute the forces acting on the cylinders' surface, we use the cylindrical stress tensor:

$$
\left[\begin{array}{ll}
\tau_{r} & \tau_{r \theta} \\
\tau_{\theta r} & \tau_{\theta \theta}
\end{array}\right]=\left[\begin{array}{cc}
-p+2 \mu \frac{\partial v_{r}}{\partial r} & \mu\left(\frac{\partial v_{\theta}}{\partial r}-\frac{v_{r}}{\theta}+\frac{1}{r} \frac{\partial v_{r}}{\partial \theta}\right) \\
\mu\left(\frac{\partial v_{\theta}}{\partial r}-\frac{v_{r}}{\theta}+\frac{1}{r} \frac{\partial v_{r}}{\partial \theta}\right) & -p+2 \mu\left(\frac{1}{r} \frac{\partial v_{\theta}}{\partial \theta}+\frac{v_{r}}{r}\right)
\end{array}\right]
$$

which reduces to

$$
\left[\begin{array}{ll}
\tau_{\pi} & \tau_{\theta \theta} \\
\tau_{\theta r} & \tau_{\theta \theta}
\end{array}\right]=\left[\begin{array}{cc}
-p(r) & \mu\left(\frac{\partial v_{\theta}}{\partial r}-\frac{v_{\theta}}{r}\right) \\
\mu\left(\frac{\partial v_{\theta}}{\partial r}-\frac{v_{\theta}}{r}\right) & -p(r)
\end{array}\right]=\left[\begin{array}{cc}
-p & -\frac{2 \mu \beta}{r^{2}} \\
-\frac{2 \mu \beta}{r^{2}} & -p
\end{array}\right]
$$

after using (3.10) and substituting (3.12). The same procedure as in section 3.1 holds to evaluate the forces, except this time we use the cylindrical coordinate system:

$$
\mathbf{F}(r)=\left\{\begin{array}{l}
F_{r} \\
F_{\theta}
\end{array}\right\}=\int_{\Gamma}\left[\begin{array}{cc}
-p(r) & -2 \mu \beta / r^{2} \\
-2 \mu \beta / r^{2} & -p(r)
\end{array}\right]\left\{\begin{array}{l}
n_{r} \\
n_{\theta}
\end{array}\right\}
$$


On the inner cylinder, $r=r_{1}, n_{r}=-1$. and $n_{\theta}=0$ :

$$
F_{1}=\int_{r_{1}}\left[\begin{array}{cc}
-p\left(r_{1}\right) & -2 \mu \beta / r_{1}^{2} \\
-2 \mu \beta / r_{1}^{2} & -p\left(r_{1}\right)
\end{array}\right]\left\{\begin{array}{c}
-1 \\
0
\end{array}\right\} d s=2 \pi r_{1}\left\{\begin{array}{c}
p\left(r_{1}\right) \\
2 \mu \beta / r_{1}^{2}
\end{array}\right\}
$$

On the outer cylinder, $r=r_{2}, n_{r}=1$. and $n_{\theta}=0$ :

$$
F_{2}=\int_{r_{2}}\left[\begin{array}{cc}
-p\left(r_{2}\right) & -2 \mu \beta / r_{2}^{2} \\
-2 \mu \beta / r_{2}^{2} & -p\left(r_{2}\right)
\end{array}\right]\left\{\begin{array}{l}
1 \\
0
\end{array}\right\} d s=2 \pi r_{2}\left\{\begin{array}{c}
p\left(r_{2}\right) \\
2 \mu \beta / r_{2}^{2}
\end{array}\right\}
$$

For numerical comparison, we set

$$
\begin{aligned}
& r_{1}=\omega_{1}=1 \\
& r_{2}=-\omega_{2}=2 \\
& \rho=1 \\
& \mu=1 / \operatorname{Re}=1 / 1000
\end{aligned}
$$

With (3.21), we can compute $\alpha$ and $\beta$ using (3.15):

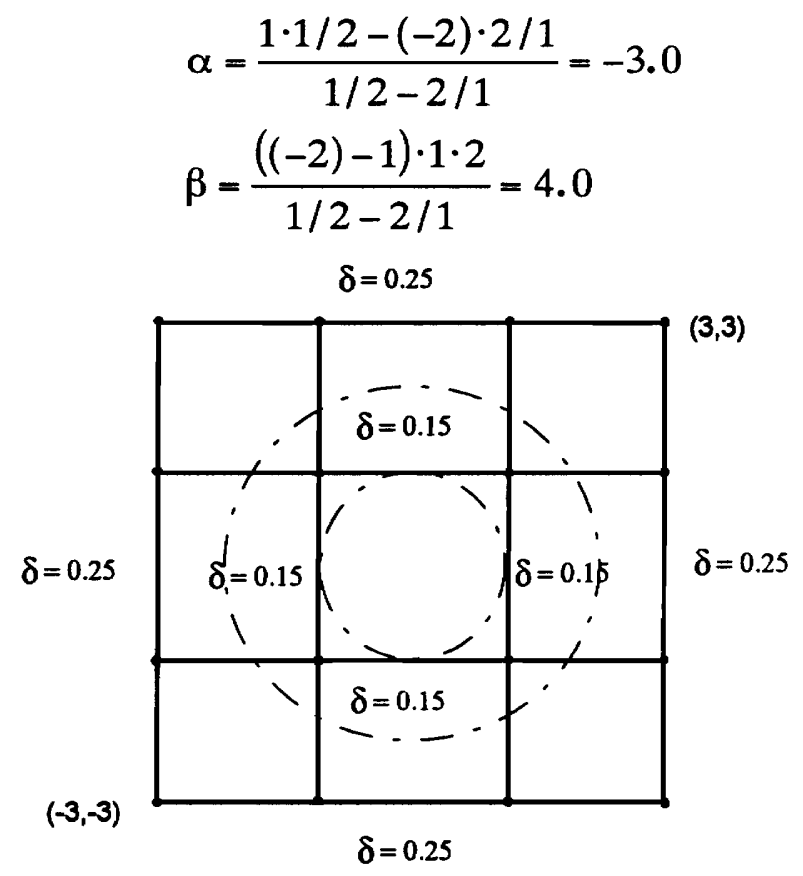

Figure 3.20 Concentric Rotating cylinder problem, First Backmesh 
a)

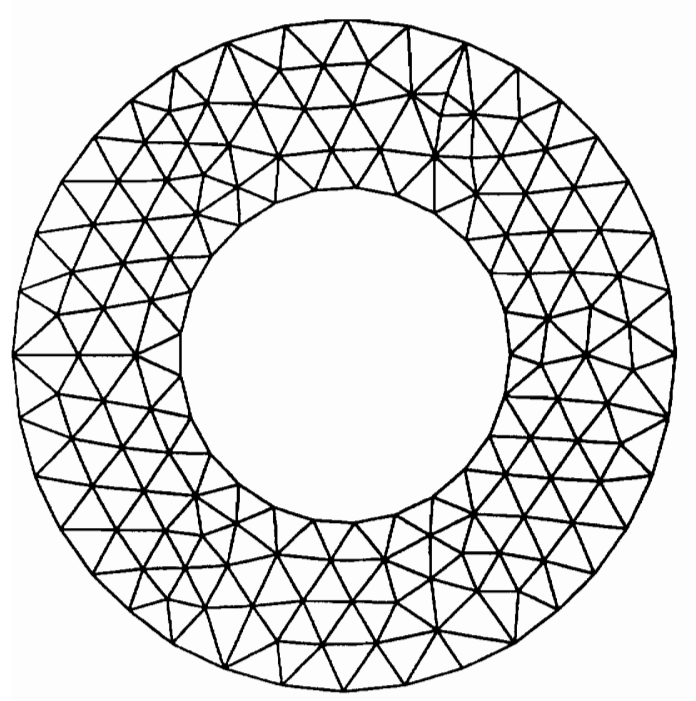

b)

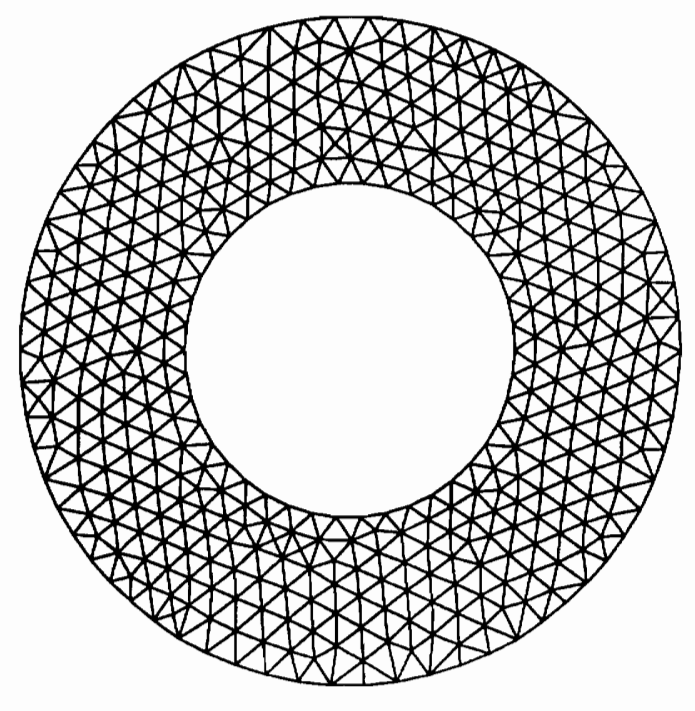

c)

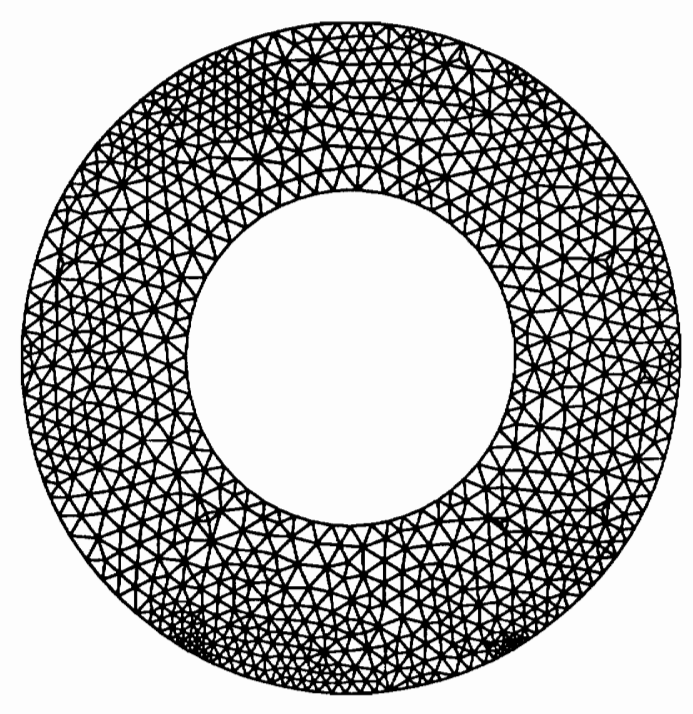

Figure 3.21 Concentric Rotating cylinders problem, Mesh Convergence

Because of the unknown constant in the pressure expression of the analytical solution, we used the projection method (cf. section 2.3) to estimate the error over the domain. Here again, we used the reduction operator for the mesh adaptivity. 


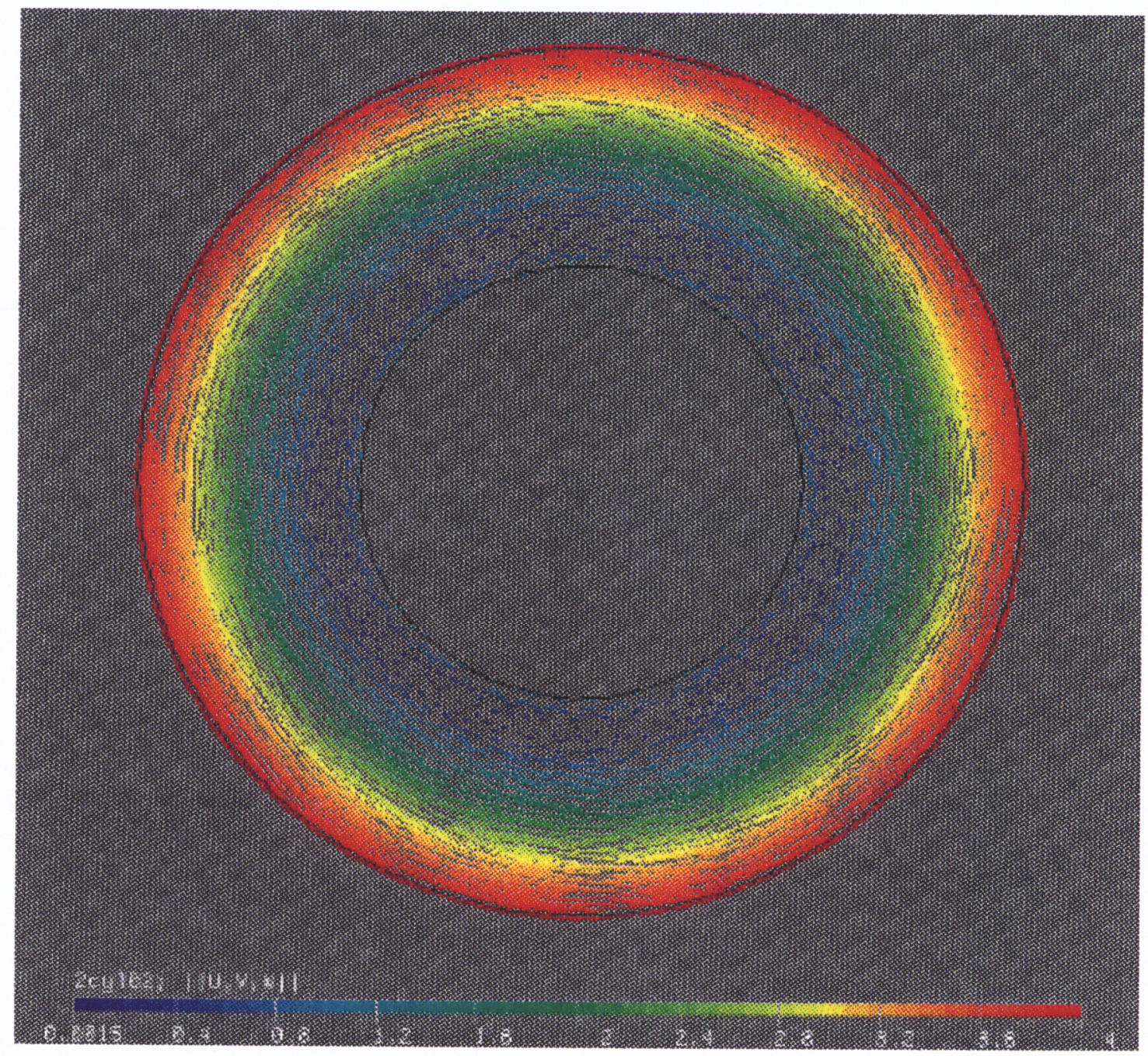
Figure 3.22 Concentric Rotating Cylinders, velocity Distribution




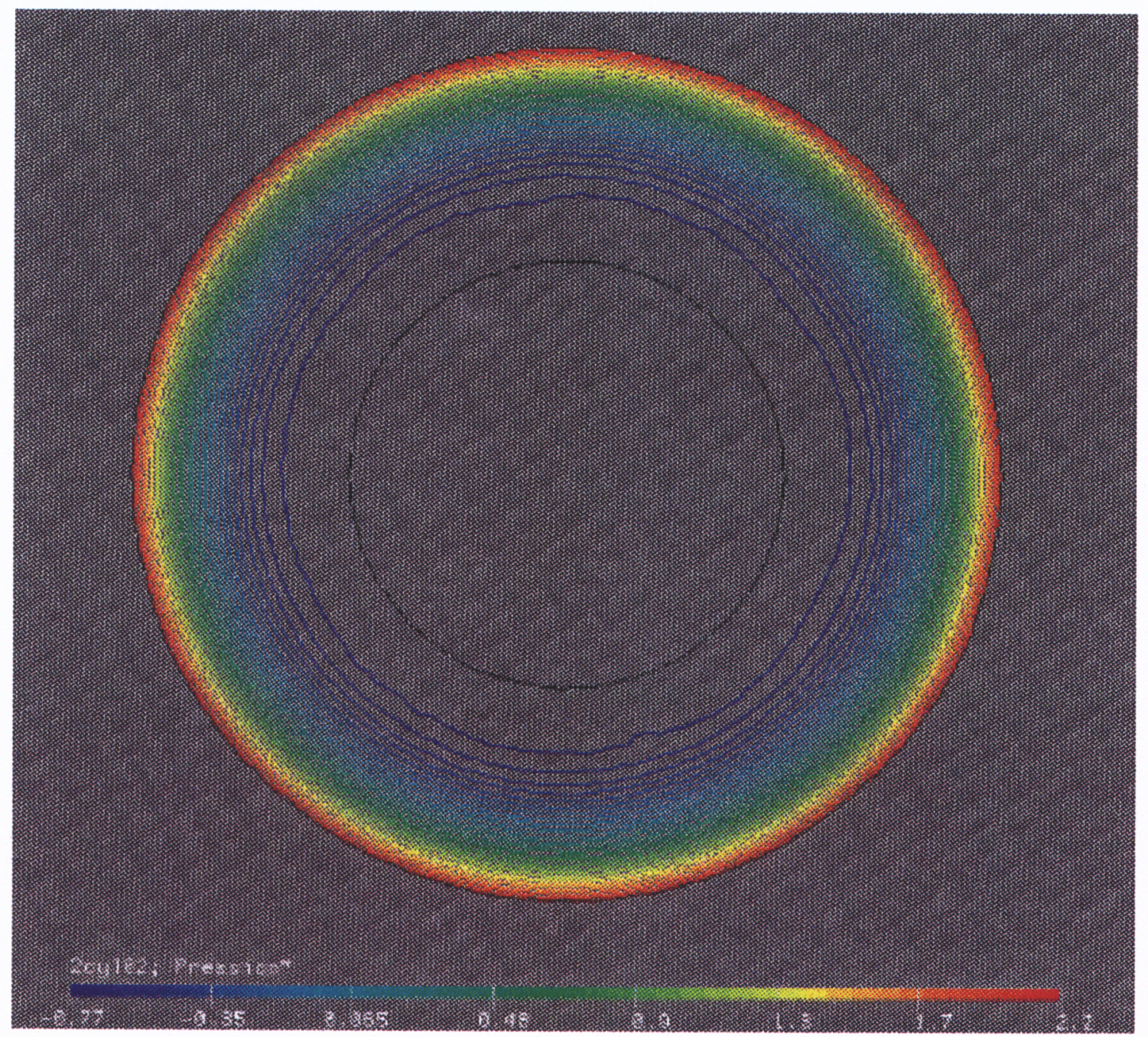

Figure 3.23 Concentric Rotating Cylinders, Pressure Distribution 
a) Cycle (a) in Figure 3.21

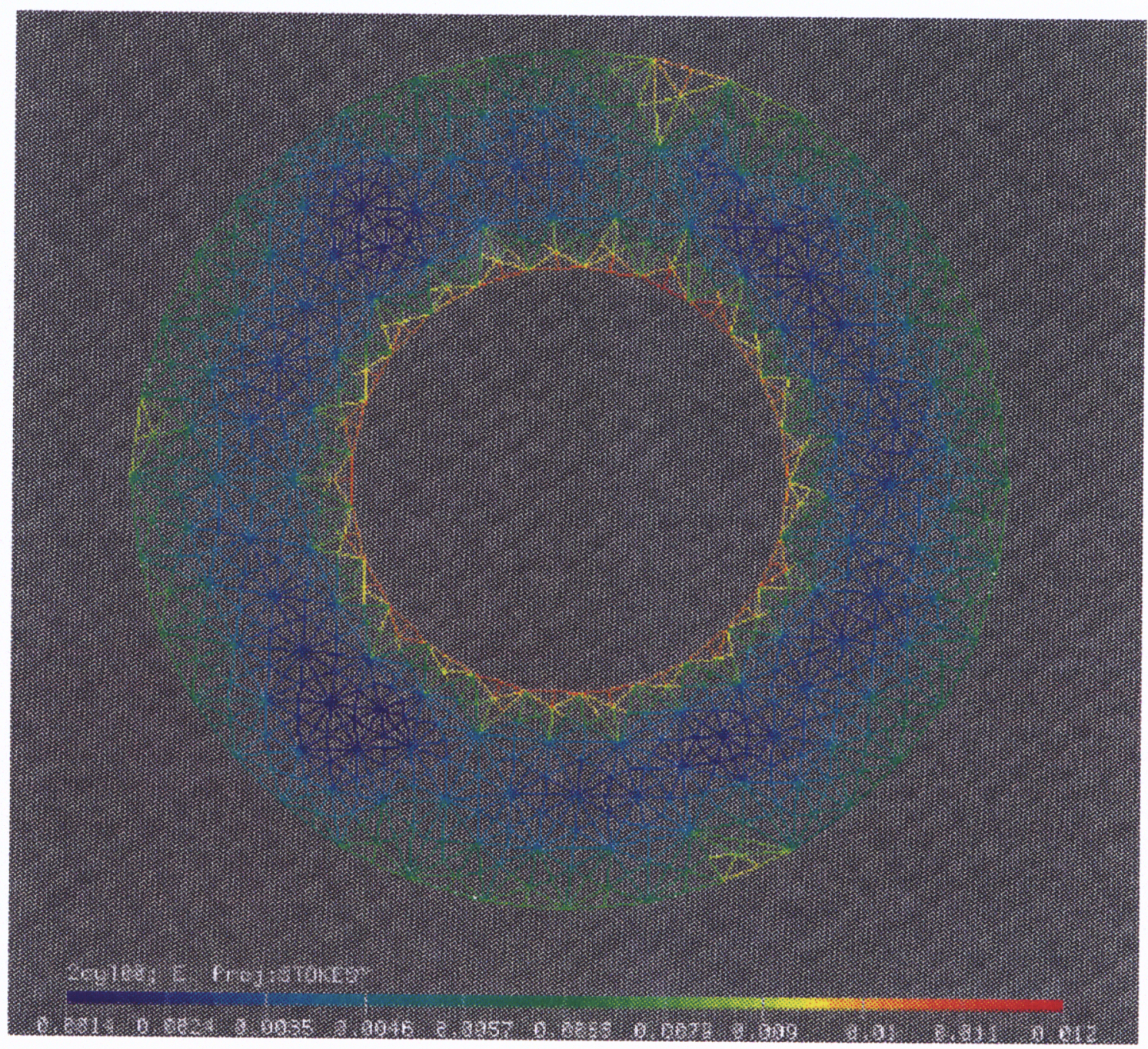

Figure 3.24 Concentric Rotating Cylinders, Error Distribution 
b) Cycle (b) in Figure 3.21

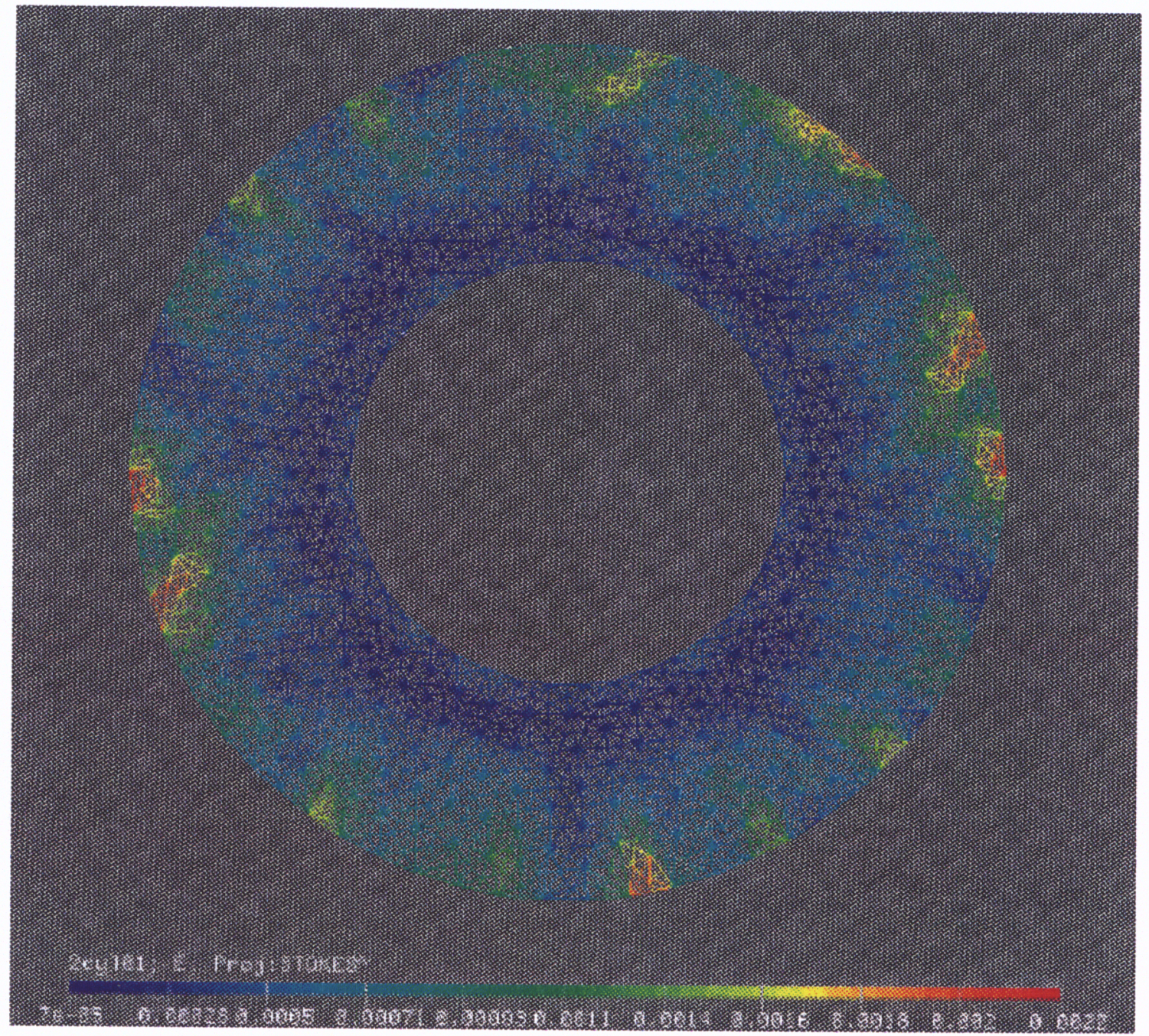

Figure 3.24 Concentric Rotating Cylinders, Error Distribution 
It is interesting to note that the target for the relative error has been reached for both cycles and that is probably because the error was quite small to start with. Also of interest is the uniformity of the three grids. This is explained by the fact that the solution is quite uniform over the domain with no rapid variation of either the velocity or the pressure (see figures 3.22 and 3.23).

Table 3.5 Concentric Rotating Cylinders, Adaptivity Strategy

\begin{tabular}{|c|c|c|c|c|c|}
\hline Cycle & $\begin{array}{c}\text { Absolute Error } \\
\text { Stokes norm }\end{array}$ & $\begin{array}{c}\text { Solution } \\
\text { Stokes norm }\end{array}$ & $\begin{array}{c}\text { Relative } \\
\text { Error }\end{array}$ & $\begin{array}{c}\text { Transition } \\
\text { Operator }\end{array}$ & $\begin{array}{c}\text { Target Rel. } \\
\text { Error }\end{array}$ \\
\hline \hline (a) & $9.040 \mathrm{e}-02$ & $3.167 \mathrm{e}+00$ & $2.85 \mathrm{e}-02$ & reduction & 0.25 \\
\hline (b) & $1.928 \mathrm{e}-02$ & $2.670 \mathrm{e}+00$ & $7.22 \mathrm{e}-03$ & reduction & 0.45 \\
\hline (c) & $8.502 \mathrm{e}-03$ & $2.679 \mathrm{e}+00$ & $3.17 \mathrm{e}-03$ & & \\
\hline
\end{tabular}

Both velocity and pressure distribution of the final solution show no variation in the angular direction. This confirms the assumption of radial symmetry in (3.11). Even though the error distribution shown in figure 3.24 presents some areas of larger error, the adaptative module doesn't concentrate elements drastically in any particular region because of the uniformity of the solution.

To compare the numerical and analytical solutions for the forces acting on the rotating cylinders, we must convert the Cartesian forces to the normal-tangential system, which is equivalent to $\langle r, \theta\rangle$ in this case. The normal component contains only pressure effects and the tangential component only viscous effects. Because the constant in the pressure expression is difficult to evaluate, we take the pressure out of the tensor expression in order to consider only viscous effects. The normal component therefore should be zero.

Table 3.6 Comparison between analytical results and f.e.m. solution for the two co-axial rotating cylinders.

\begin{tabular}{|c||c|c|c|c|c|}
\cline { 2 - 6 } \multicolumn{1}{c|}{} & \multicolumn{2}{c|}{ Analytical Solution } & \multicolumn{2}{c|}{ Finite Element Method } & $\begin{array}{c}\text { Relative } \\
\text { Error }\end{array}$ \\
\hline Cylinder & $\mathrm{F}_{\mathrm{t}}$ & $\mathrm{F}_{\mathrm{n}}$ & $\mathrm{F}_{\mathrm{t}}$ & $\mathrm{F}_{\mathrm{n}}$ & $\mathrm{e}_{\mathrm{FI}}$ \\
\hline \hline Inner & -.05026548 & 0.0 & -0.0488874 & $.86424 \mathrm{e}-03$ & $2.726 \mathrm{e}-02$ \\
\hline Outer & .025132741 & 0.0 & 0.0250085 & $-.52099 \mathrm{e}-03$ & $4.727 \mathrm{e}-03$ \\
\hline
\end{tabular}

This problem ends the validation section and demonstrate the ability of the finite element methodology to handle more complex flows such as a rotating annulus. 


\subsection{Flat Plate Airfoil with Rotating L.E. Cylinder}

With the previous problems, we verified the validity of the finite-element method to compute the forces exerted on a surface by a moving fluid. We tested its capability to solve a full Navier-Stokes (with Boussinesq approximation) problem in a simple case first and then in more realistic cases such as plate of non-negligible thickness in flows of various viscosities. We also studied the flow behavior in a rotating annulus and compared the viscous effects in the aerodynamic forces between the analytical solution and the finiteelement results. We are now ready to begin the analysis of a uniform flow about a cylinder rotating in front of an angled flat plate.

\subsubsection{Discretization}

The problem geometry and boundary-conditions are shown in figure 3.25. The non dimensional geometrical parameters are described in table 3.7.

Table 3.7 Flat plate and cylinder geometrical dimensions

\begin{tabular}{|c|c|}
\hline Quantity & dimension \\
\hline \hline $\mathrm{c}$ & 1.0 \\
\hline $\mathrm{d} / \mathrm{c}$ & 0.075 \\
\hline $\mathrm{h} / \mathrm{c}$ & 0.05 \\
\hline $\mathrm{e} / \mathrm{c}$ & 0.01 \\
\hline $\mathrm{t} / \mathrm{c}$ & 0.2 \\
\hline
\end{tabular}

All dimensions are based on the chord length. The plate and cylinder geometry was first derived from Modi \& Fernando's ${ }^{28}$ study of a flat plate with rotating cylinders at both leading and trailing edges $(d / c \cong 0.3, h / c \cong 0.1)$. After a few tests, it appeared that those dimensions were applied in a flow of relatively low viscosity $\left(\mathbf{R e}=3 \times 10^{4}\right)$ and that their size should be reduced for a flow of higher viscosity $(d / c \cong 0.075, h / c \cong 0.05)$. It was also decided to modify the shape of the trailing edge for a smoother flow around that edge. Modi \& Fernando make no mention of the gap size but in a later study on an aircraft ${ }^{14}$, Modi \& al. set the gap size to one percent of the chord. The external boundaries correspond to a length-width ratio of twenty percent, that is the wind tunnel section used in Modi's flat plate experiment. The plate is situated in the flow such that it remains approximately centered when the angle of attack increases. The fact that the plate is not precisely centered in the computational domain will produce some important effects on our results.

Along with the geometry, we must provide the initial backmesh to define the finite element problem. This task is more difficult than for the previous problems because of the complex 
geometry involved. There is no fixed method to construct the most efficient initial backmesh, the usual procedure relies on trial and error until a satisfactory result is obtained. The first step consist in locating the points on the geometry that require more precision in the discretization and construct the triangles that will define the coarse grid.

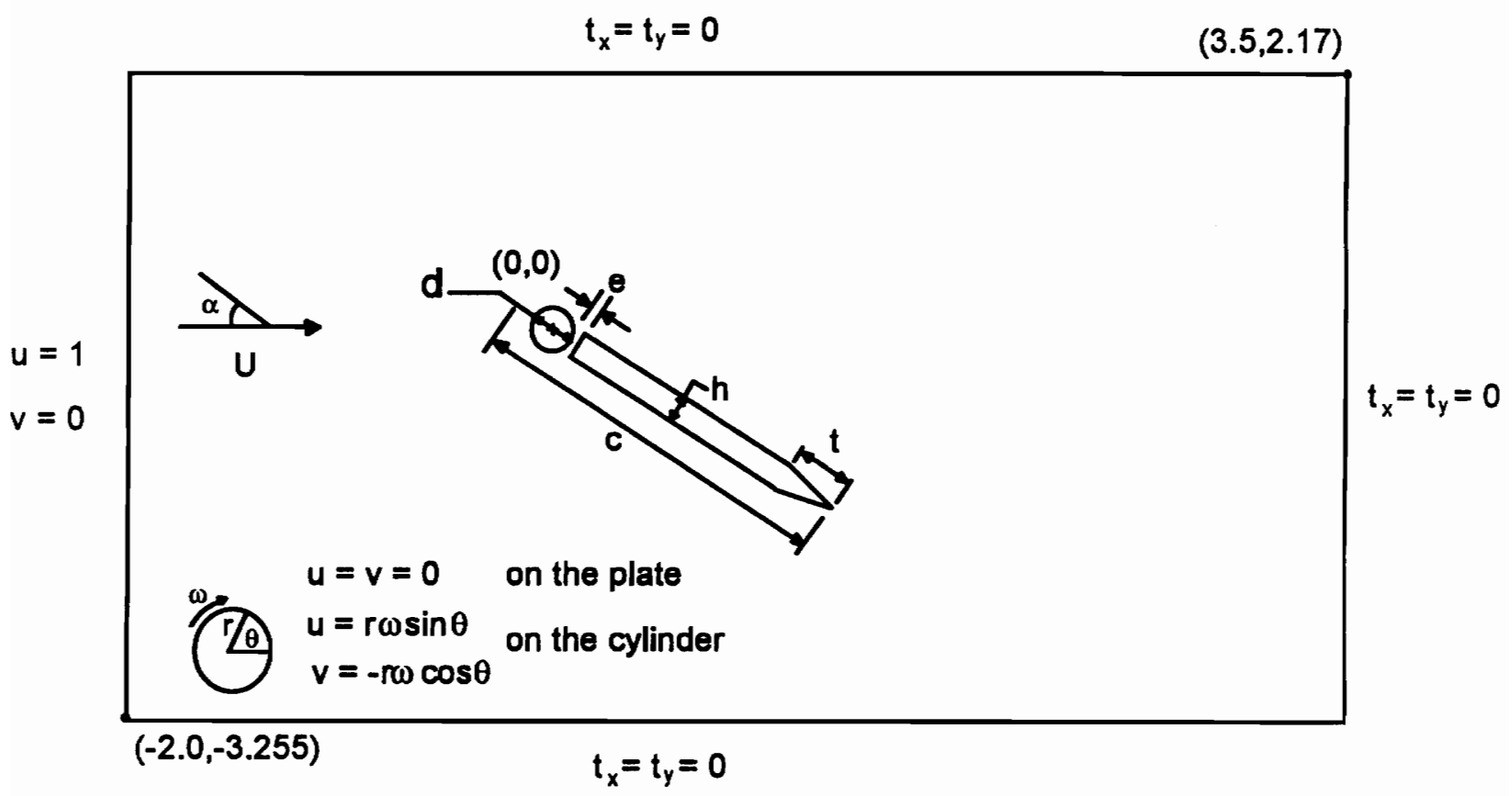

Figure 3.25 Flat Plate with Rotating L.E. Cylinder Problem, Geometry

In the second step, we have to impose values for the element size function $\delta$ on each node. The best guess for the element size $\delta$ is to use the smallest dimension of the problem geometry in the area of the node. According to the grid produced by the mesh generator, the element size function may (most probably!) have to be modified if there are too many or too few elements in some areas of the domain.

Each time we vary the angle of attack, the geometry changes along with the initial backmesh. Because the procedure described above is time consuming, a little program has been created to automatically compute the geometry and the initial backmesh for a given angle of attack. Figure 3.26 shows the initial backmesh as a function of the angle of attack $\alpha$. We give fixed values for the element size function since the dimensions of the plate and cylinder are constant from one angle to another. These values can be adjusted subsequently according to the amount of precision in the discretization needed as the complexity of the flow increases. 


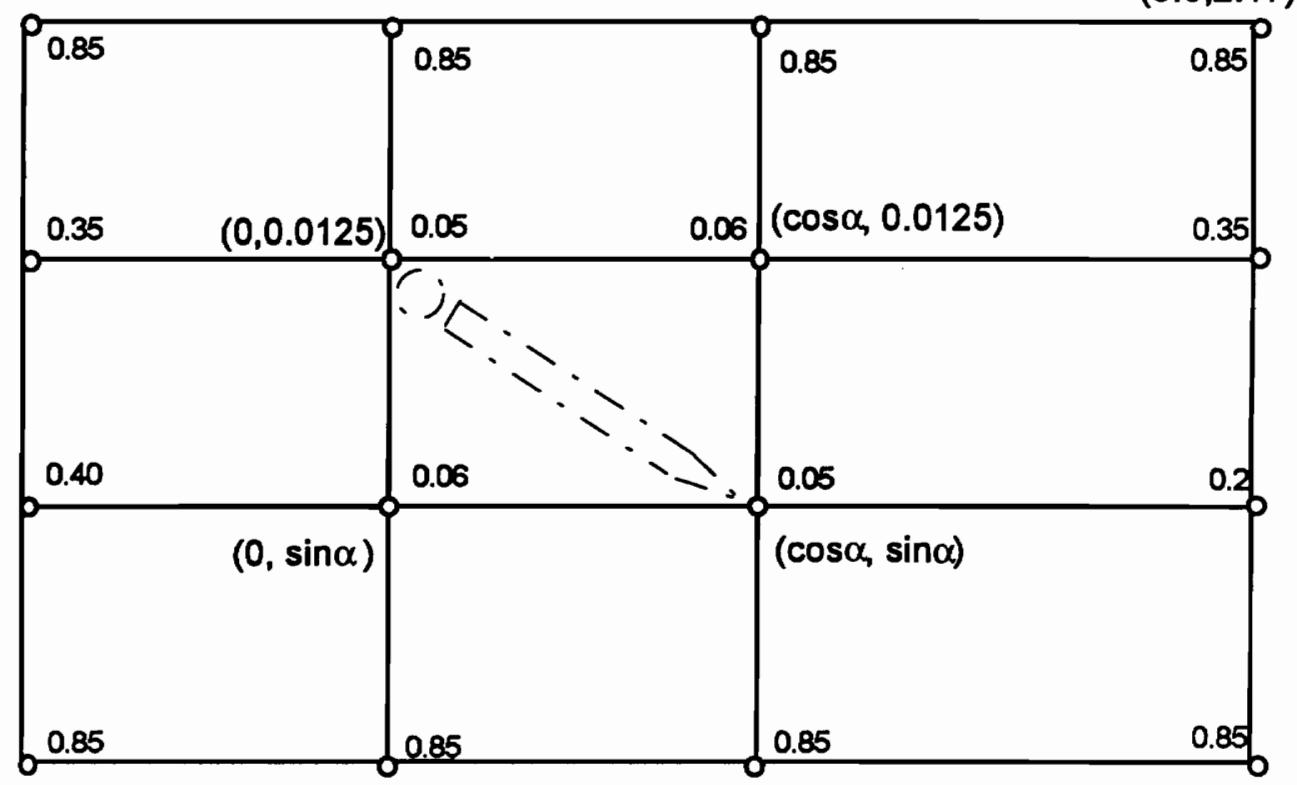

$(-2.0,-3.255)$

Figure 3.26 Flat Plate with Rotating L.E. Cylinder, Initial Backmesh

\subsubsection{Resolution}

Starting with $\alpha=5^{\circ}$, the first approximate solution is obtained from zero initial conditions. Those are fairly poor initial conditions if we want to solve the flow at a relatively high Reynolds number. For this reason, we have to start the computation at very low Reynolds number and slowly increase it to the desired value of 1000 . We call this process a continuation in Reynolds number. For example, after obtaining a first solution at $\operatorname{Re}=100$ from zero initial conditions, we set $\operatorname{Re}=300$ in the next iteration and use the $\operatorname{Re}=100$ solution as initial conditions. We repeat this operation, always using the same grid, until we reach $R e=1000$. Because this process requires a lot of time and computation, we used a non-standard procedure to obtain a solution at $\mathrm{Re}=1000$ for all other angles of attack. This procedure of resolution is similar for each angle of attack. We will describe it here for a single value of angle of attack, say $\alpha=15^{\circ}$.

We started from $\alpha=5^{\circ}$ and achieved a Reynolds number of a thousand as described above. Now, instead of repeating this process for $\alpha=10^{\circ}$, we directly set $\operatorname{Re}=1000$ and used a solution at $\alpha=5^{\circ}$ and $\operatorname{Re}=1000$ as initial conditions. After two adaptive 
remeshing iterations, we reached the same level of accuracy as if we had followed the normal procedure. For $\alpha=15^{\circ}$, we used a solution at $\alpha=10^{\circ}$ as initial conditions.

For every angle of attack, we first set the rotational speed of the cylinder at the lowest value possible. This value increases as we raise the angle of attack since the flow tends to separate from the plate surface. Separation generates instability in the flow and because we are assuming steady-state conditions, it becomes impossible to converge toward a unique solution. In addition, the discretization of the grid generated from the initial backmesh is usually not accurate enough to capture all the complexity of the flow. In the case that we are now studying, we have the following conditions:

$$
\begin{array}{ll}
\text { - } & \alpha=15^{\circ} \\
\text { - } & \operatorname{Re}=1000 \\
\text { - } & (r \cdot \omega) / U_{\infty}=0.1
\end{array}
$$

Because of the non-usual procedure described above, the strategy used to adapt the mesh differs from the previous examples. After a few tests, we concluded that the most effective strategy is to use the fixed target operator for the first adaptive cycle and to set the target approximately to the value obtained for the relative error in the previous angle solution. Then we switch to the reduction operator and request a $50 \%$ reduction of the error. Table 3.8 presents the discretization characteristics of the $\alpha=10^{\circ}$ solution.

Table 3.8 Flat Plate with Rotating L.E. Cylinder, error analysis, $\alpha=10^{\circ}$

\begin{tabular}{|c|c|c|c|}
\hline Cycle & \# Nodes & \# Elements & Relative Error \\
\hline \hline (a) & 2386 & 1155 & $4.91 \mathrm{e}-02$ \\
\hline (b) & 4524 & 2211 & $1.44 \mathrm{e}-02$ \\
\hline (c) & 4636 & 2263 & $7.19 \mathrm{e}-03$ \\
\hline
\end{tabular}

If we use the most accurate solution (cycle (c)) at $\alpha=10^{\circ}$ as initial conditions, the accuracy requirements for the solution at $\alpha=15^{\circ}$ will be too high and we won't be able to obtain the target fixed in the adaptive strategy. It was found more effective to use the solution with a moderate level of accuracy (cycle (b)) instead. The adaptive strategy is resumed in table 3.9 and the mesh convergence is shown in figure 3.27 . 
Table 3.9 Flat Plate with Rotating L.E. Cylinder, Adaptivity Strategy

\begin{tabular}{|c|c|c|c|c|c|}
\hline Cycle & $\begin{array}{c}\text { Absolute Error } \\
\text { Stokes norm }\end{array}$ & $\begin{array}{c}\text { Solution } \\
\text { Stokes norm }\end{array}$ & $\begin{array}{c}\text { Relative } \\
\text { Error }\end{array}$ & $\begin{array}{c}\text { Transition } \\
\text { Operator }\end{array}$ & $\begin{array}{c}\text { Target Rel. } \\
\text { Error }\end{array}$ \\
\hline \hline (a) & $8.195 \mathrm{e}-03$ & $3.072 \mathrm{e}-01$ & $2.67 \mathrm{e}-02$ & fixed target & 0.01 \\
\hline (b) & $3.822 \mathrm{e}-03$ & $3.002 \mathrm{e}-01$ & $1.27 \mathrm{e}-02$ & reduction & 0.40 \\
\hline (c) & $1.647 \mathrm{e}-03$ & $3.023 \mathrm{e}-01$ & $5.45 \mathrm{e}-03$ & & \\
\hline
\end{tabular}

(a)

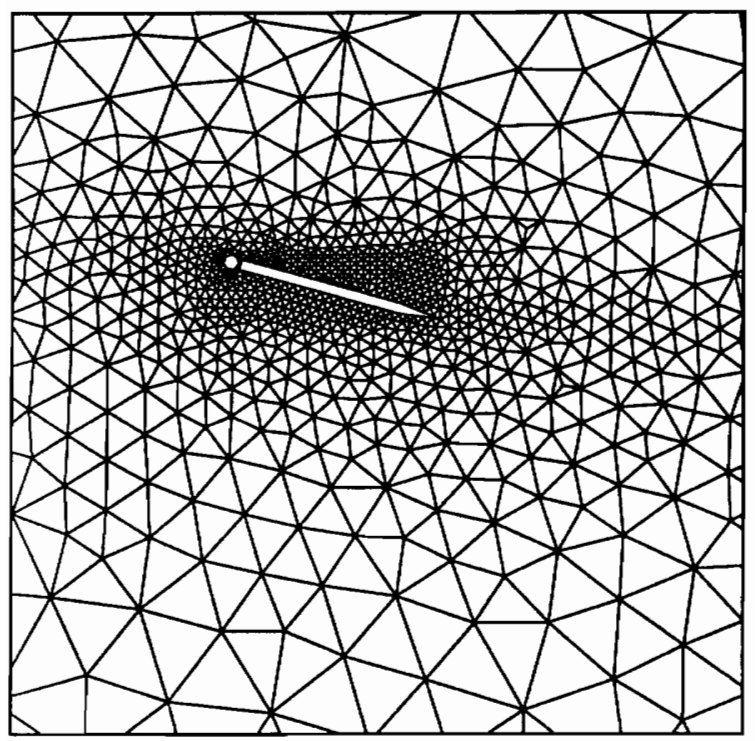

(b)

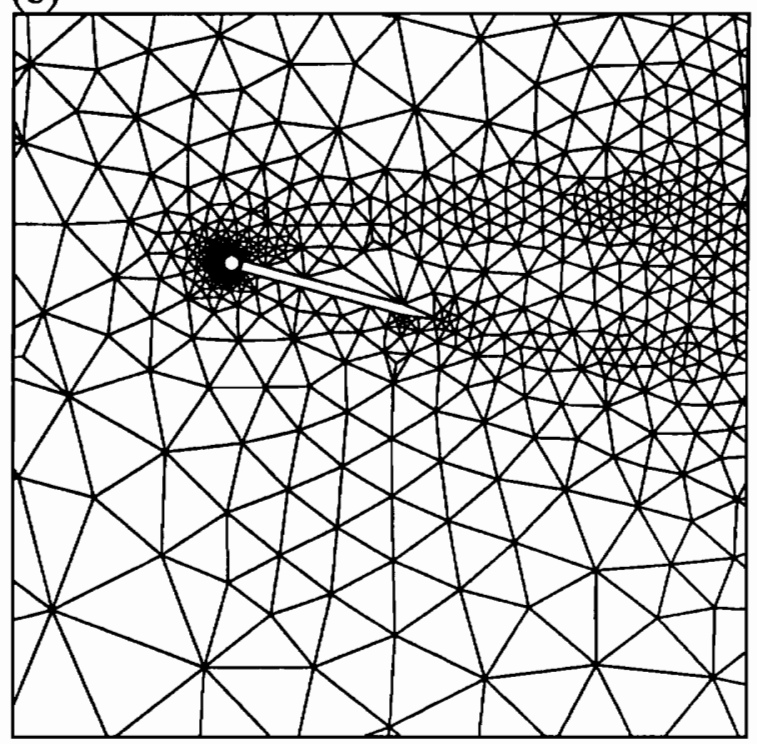

(c)

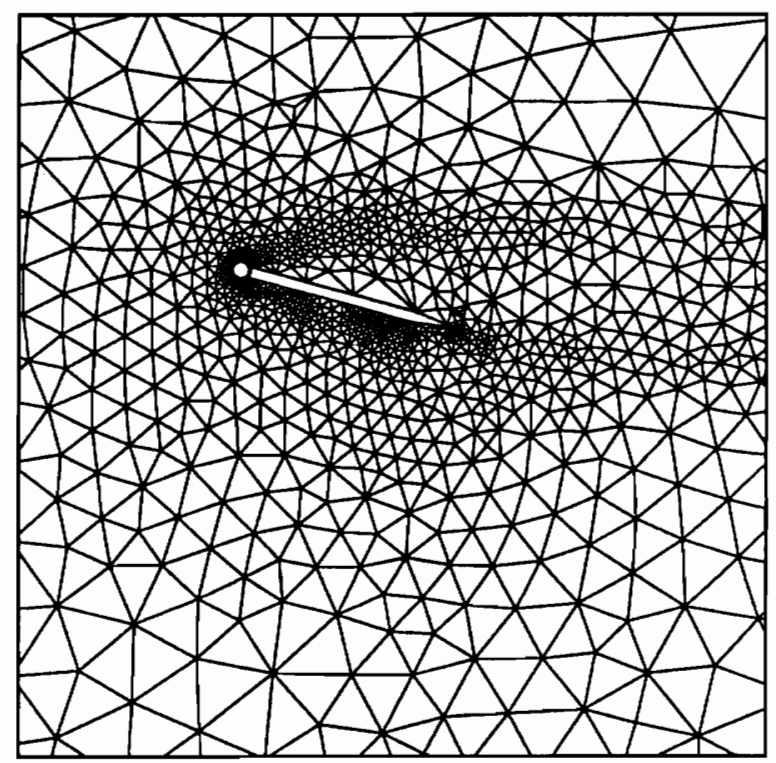

Figure 3.27 Flat Plate with Rotating L.E.Cylinder, $\alpha=15^{\circ}$, Mesh Convergence 
The first grid (figure 3.27 (a)) corresponds to the element size function defined on the initial backmesh. We see in figure 3.31 a) that the error is mostly concentrated around the cylinder on this mesh. The fixed target operator detects that error concentration and generates many small elements around the cylinder (figure 3.27 (b)). It also locates error in the wake behind the plate, thus we observe more elements in that region. The large elements under the plates can probably be caused by the fact that we used a solution at a smaller angle as initial conditions. The flow right under the plate at $\alpha=15^{\circ}$ is a less critical region for $\alpha=10^{\circ}$. Figure $3.31 \mathrm{~b}$ ) confirms this fact as we see that the biggest error is located under the plate. This phenomenom is corrected in the final mesh (figure 3.27 (c)) were we see that the reduction operator concentrated many small elements in that area. The wake is also more sharply defined. The large elements right over the plate come from the fact that the velocity in that region is quite small, and so is the estimated error. In that same mesh, the error (however pretty small) is located in the upper right corner of the domain since the reduction operator increased the element size in that area. Keeping in mind that the interest of this study is to compute the aerodynamic forces acting on the cylinder-plate system, the important fact is that the error is pretty small $(\mathrm{O}(\mathrm{e}-06))$ in that area. Figure 3.28 present in greater details the discretization around the plate.

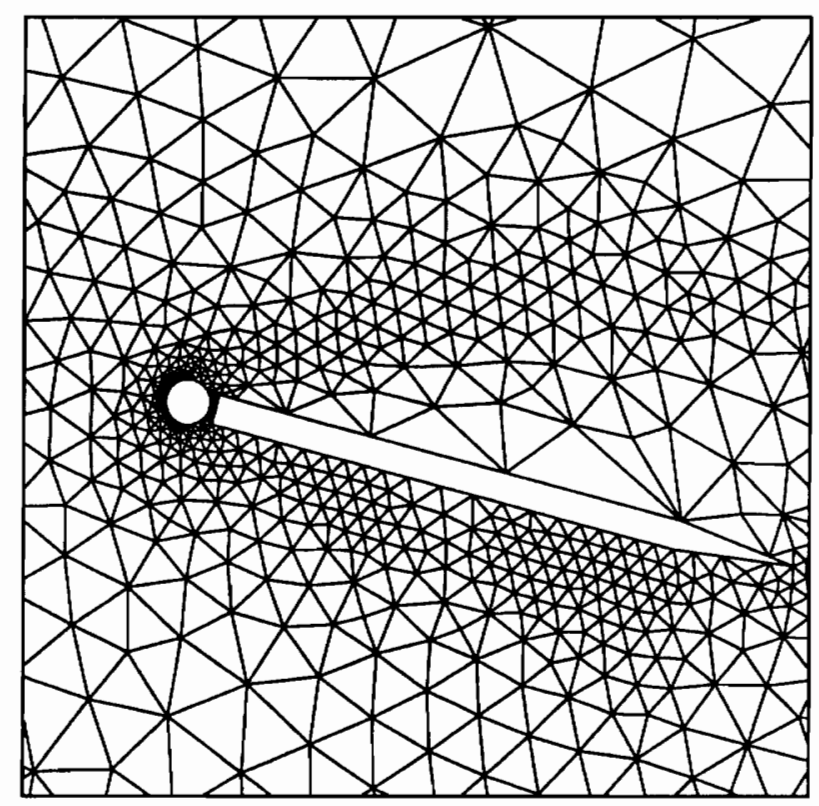

Figure 3.28 Flat Plate with Rotating L.E. Cylinder, $\alpha=15^{\circ}$, Mesh Detail of figure 3.27 (c) 
Velocity and pressure distribution for the final mesh are shown in figures 3.29 and 3.30. We can observe in figure 3.29 a recirculation zone above the plate. We will see, as we increase the rotational speed of the cylinder, this feature disappears and the wake region narrows down. The pressure distribution presents high gradients in the cylinder area. Also observed in the velocity distribution, those high gradients explain the need for a very fine discretization in the cylinder region.

Once we have an optimal grid for the flow resolution, we keep this same mesh and use it to generate solutions at different cylinder rotational speeds. The value of $\omega$ is gradually increased by modifying the cylinder boundary conditions at every iteration, each one using the preceding solution as initial conditions. Table 3.10 presents the aerodynamic forces on the system plate-cylinder for $0.0<\omega<4.0$.

Table 3.10 Flat Plate with Rotating L.E. Cylinder, $\alpha=15^{\circ}, \operatorname{Re}=1000$, Aerodynamic Forces

\begin{tabular}{|c|c|c|c|}
\hline$(r \cdot \omega) / U_{\infty}$ & Lift $(L)$ & $\operatorname{Drag}(\mathrm{D})$ & $(\mathrm{L} / \mathrm{D})=(\mathrm{Cl} / \mathrm{Cd})$ \\
\hline \hline 0.0 & 0.208064 & 0.111449 & 1.866899 \\
\hline 0.1 & 0.214153 & 0.109851 & 1.949486 \\
\hline 0.5 & 0.246472 & 0.103270 & 2.386676 \\
\hline 1.0 & 0.311805 & $0.962783 \mathrm{e}-01$ & 3.238580 \\
\hline 1.5 & 0.396791 & $0.921909 \mathrm{e}-01$ & 3.467707 \\
\hline 2.0 & 0.477312 & $0.922896 \mathrm{e}-01$ & 5.171894 \\
\hline 2.5 & 0.521081 & $0.924338 \mathrm{e}-01$ & 5.637343 \\
\hline 3.0 & 0.542160 & $0.912560 \mathrm{e}-01$ & 5.941089 \\
\hline 3.5 & 0.555091 & $0.896236 \mathrm{e}-01$ & 6.193581 \\
\hline 4.0 & 0.564291 & $0.878146 \mathrm{e}-01$ & 6.425936 \\
\hline
\end{tabular}

We see from this table that the moving wall boundary-layer control efficiently increases the lift and reduces the drag of the plate-cylinder system. For a fifteen degree angle of attack, Modi \& Fernando2s obtained the results in table 3.11 for $\mathrm{Re}=30000$. 


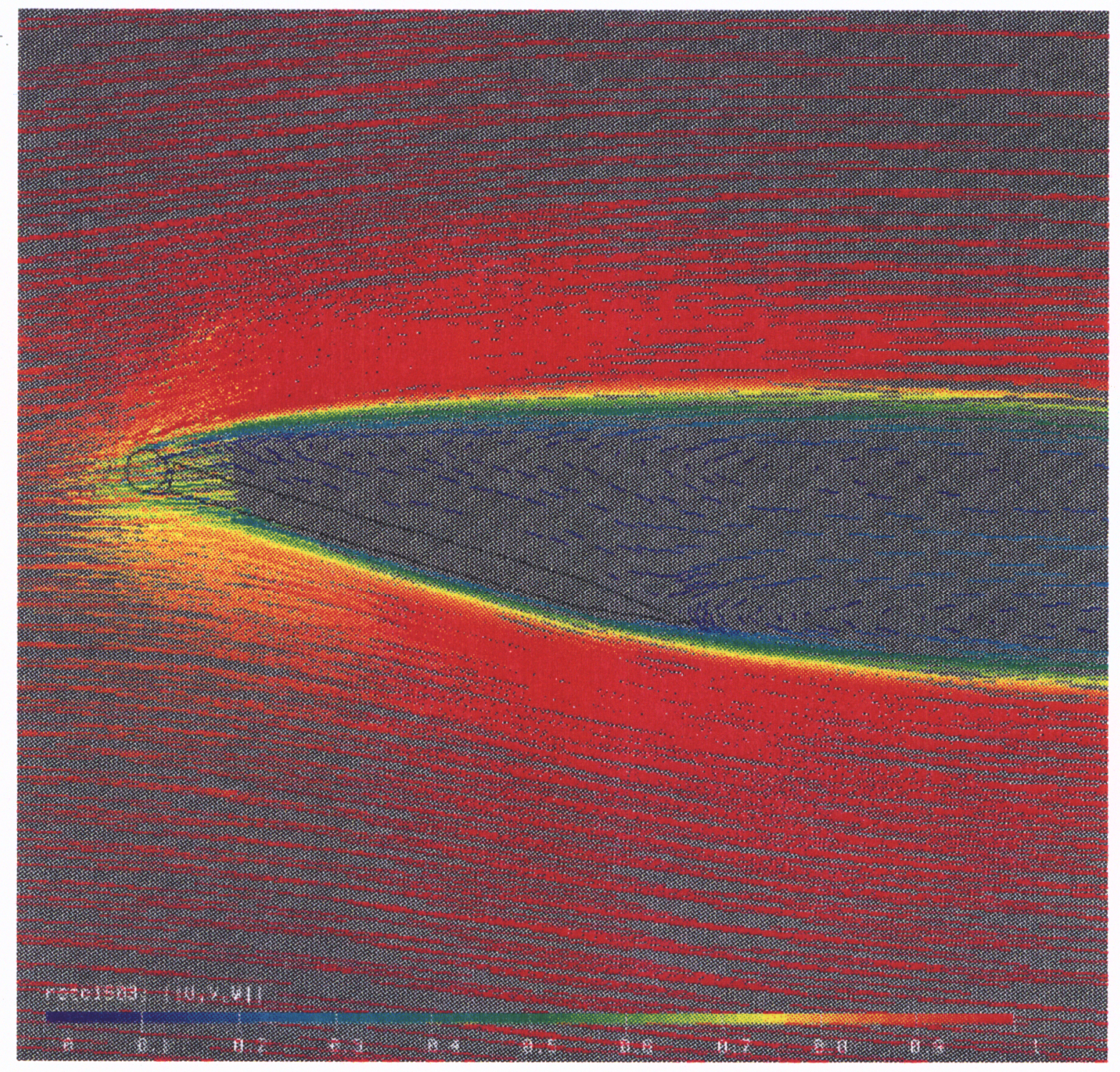

Figure 3.29 Flat Plate with Rotating L.E. Cylinder, Alpha $=15,\left(r^{*} \mathrm{~W}\right) / \mathrm{U}=0.1$, Velocity Distribution 
Figure 3.30 Flat Plate with Rotating L.E. Cylinder, Alpha $=15,\left(r^{*} \mathrm{~W}\right) / \mathrm{U}=0.1$, Pressure Distribution 
a) Cycle (a) in Figure 3.27

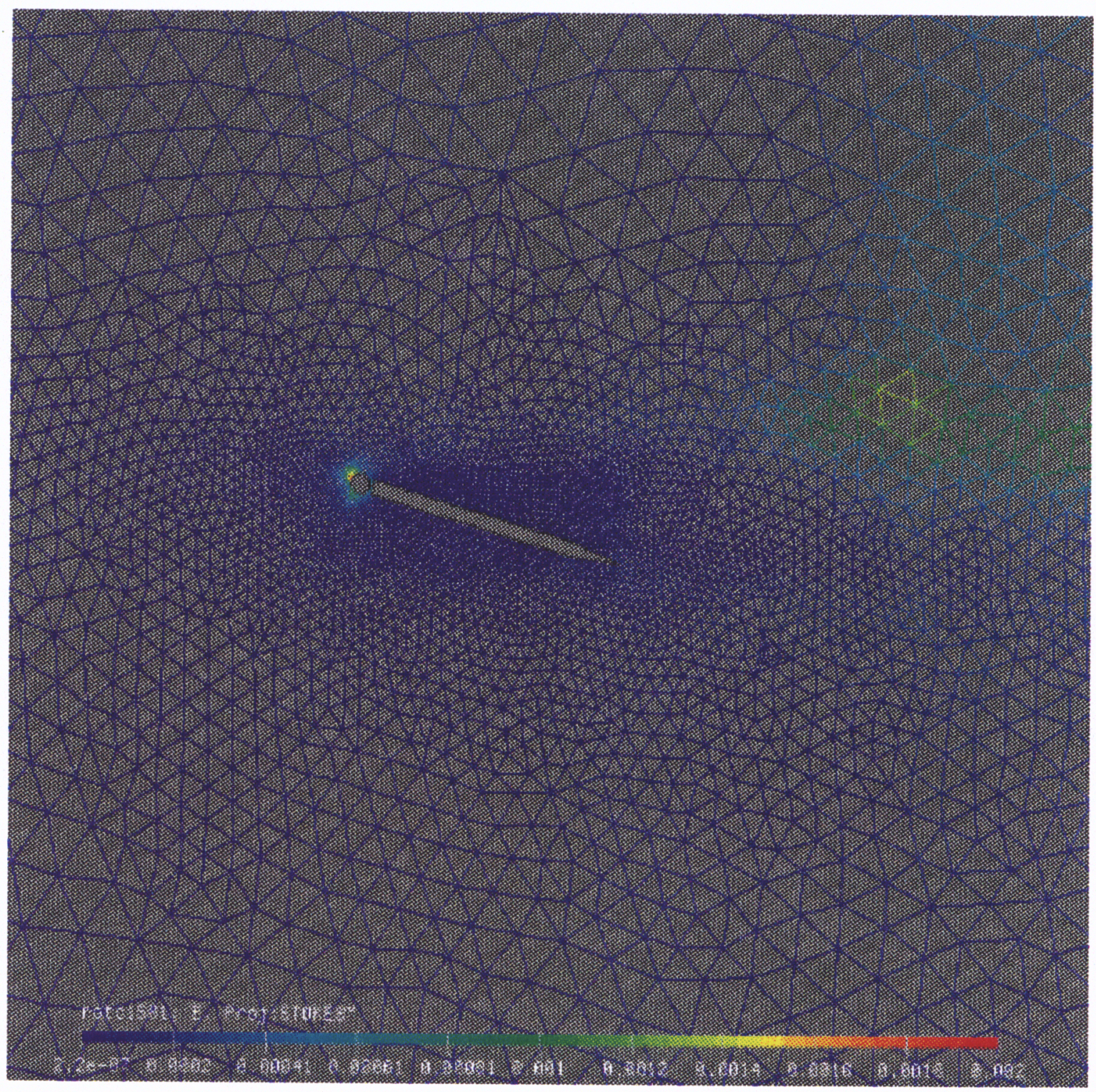

Figure 3.31 Flat Plate with Rotating L.E. Cylinder, Alpha $=15,\left(r^{*} \mathrm{w}\right) / \mathrm{U}=0.1$, Error Distribution 
b) Cycle (b) in Figure 3.27

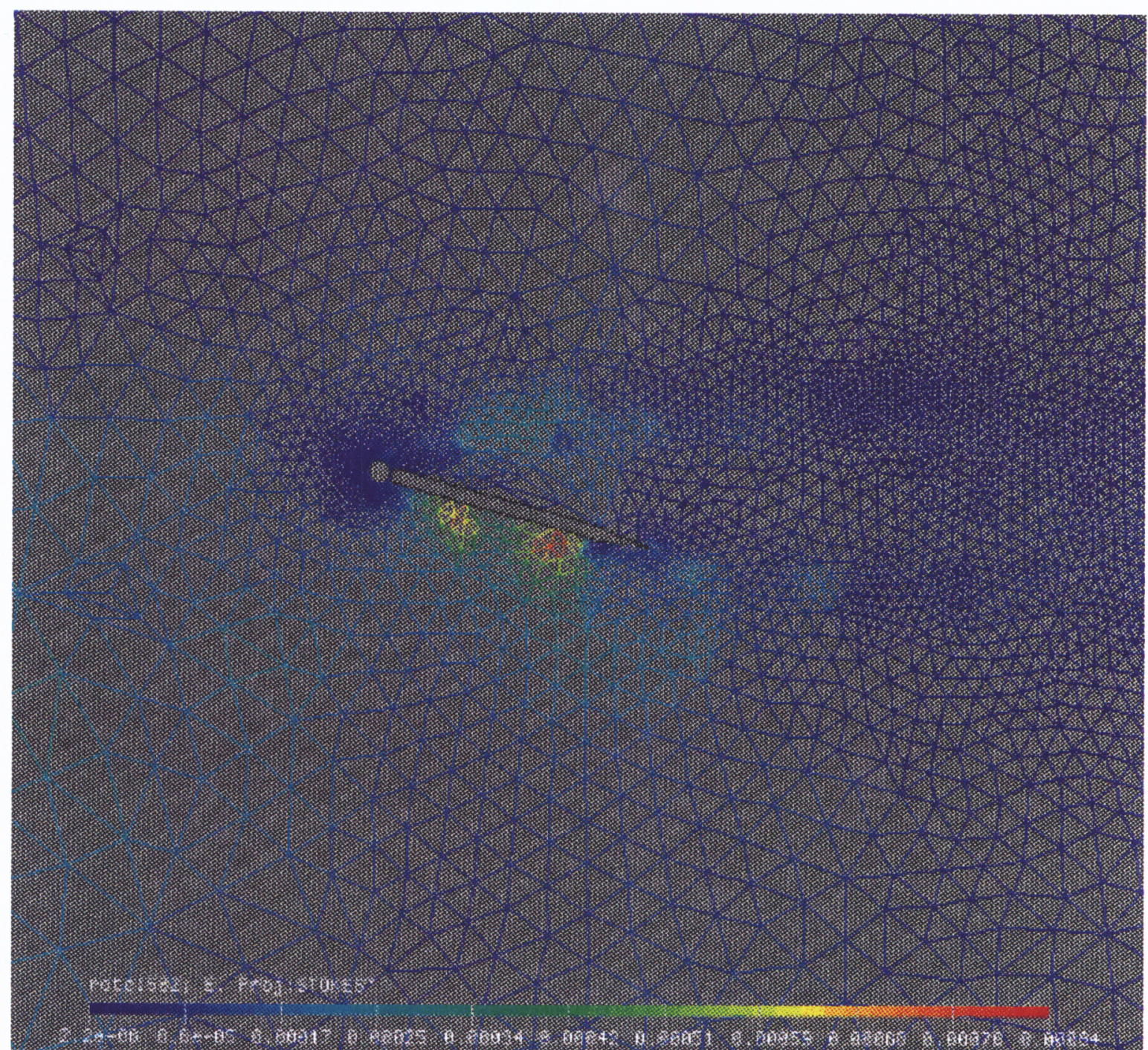

Figure 3.31 Flat Plate with Rotating L.E. Cylinder, Alpha $=15,\left(r^{*} \mathrm{~W}\right) / \mathrm{U}=0.1$, Error Distribution 
c) Cycle (c) in Figure 3.27

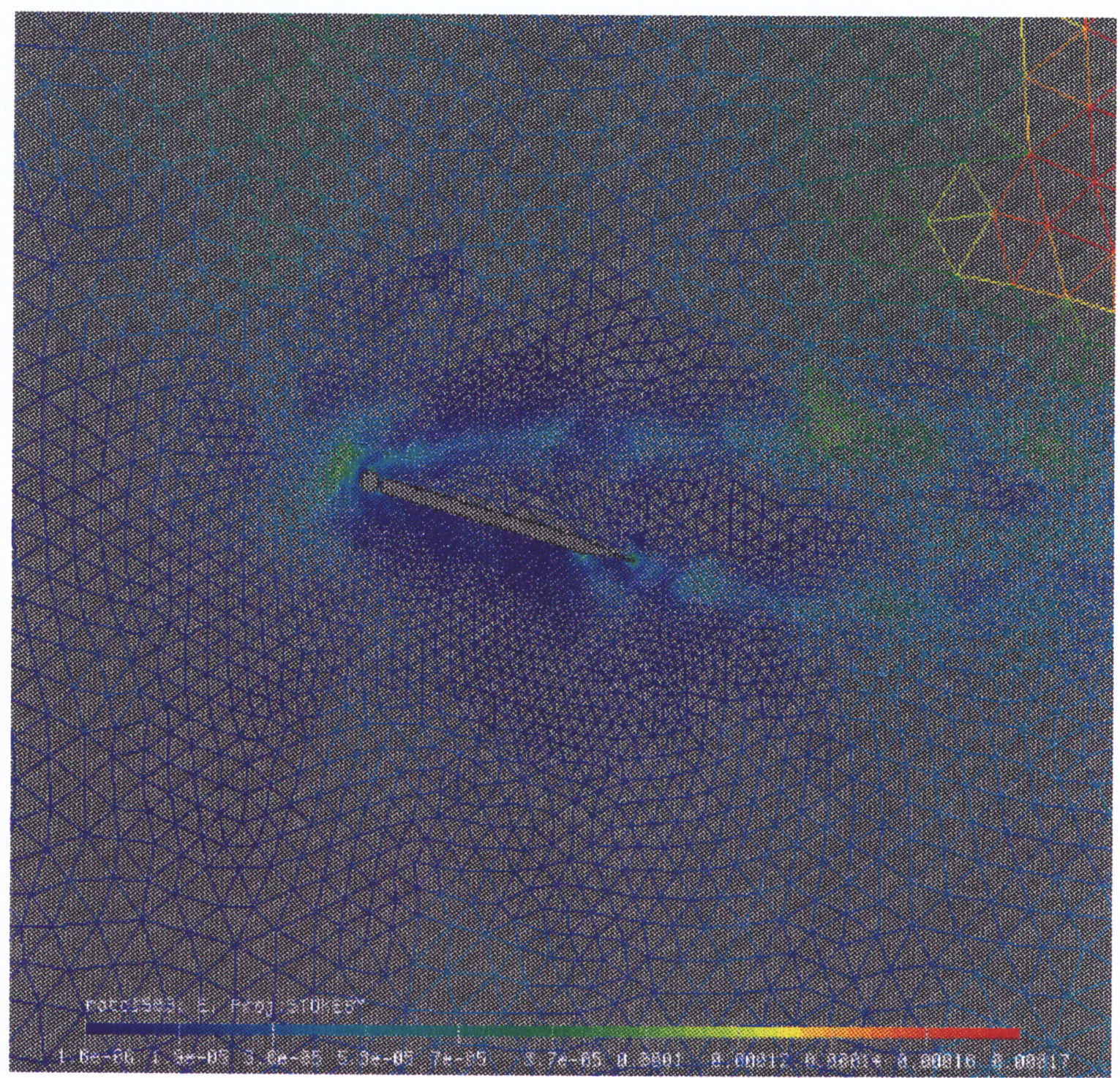

Figure 3.31 Flat Plate with Rotating L.E. Cylinder, Alpha $=15,(r * w) / U=0.1$, Error Distribution 
Table 3.11 Flat Plate with Rotating Cylinder at Leading Edge and Non-Rotating Cylinder at Trailing Edge, $\alpha=15^{\circ}, \operatorname{Re}=30000$, Aerodynamic Coefficients

\begin{tabular}{|c|c|c|c|}
\hline$(r \cdot \omega) / \mathrm{U}_{\infty}$ & $\mathrm{Cl}$ & $\mathrm{Cd}$ & $\mathrm{Cl} / \mathrm{Cd}$ \\
\hline 0.0 & 0.40 & 0.40 & 1.0 \\
\hline 1.0 & 0.95 & 0.28 & 3.4 \\
\hline 2.0 & 1.33 & 0.28 & 4.8 \\
\hline 3.0 & 1.13 & 0.22 & 5.1 \\
\hline
\end{tabular}

The two problems are somewhat similar but still pretty different in the geometry and the flow viscosity. Modi \& Fernando have two cylinders with the flat plate (only the leading edge cylinder rotating) in a flow of $\mathrm{Re}=30000$ while we have only one cylinder and a wedge trailing edge in a flow of $\mathrm{Re}=1000$. However, it is interesting to compare both results in a qualitative manner (See figure 3.32).

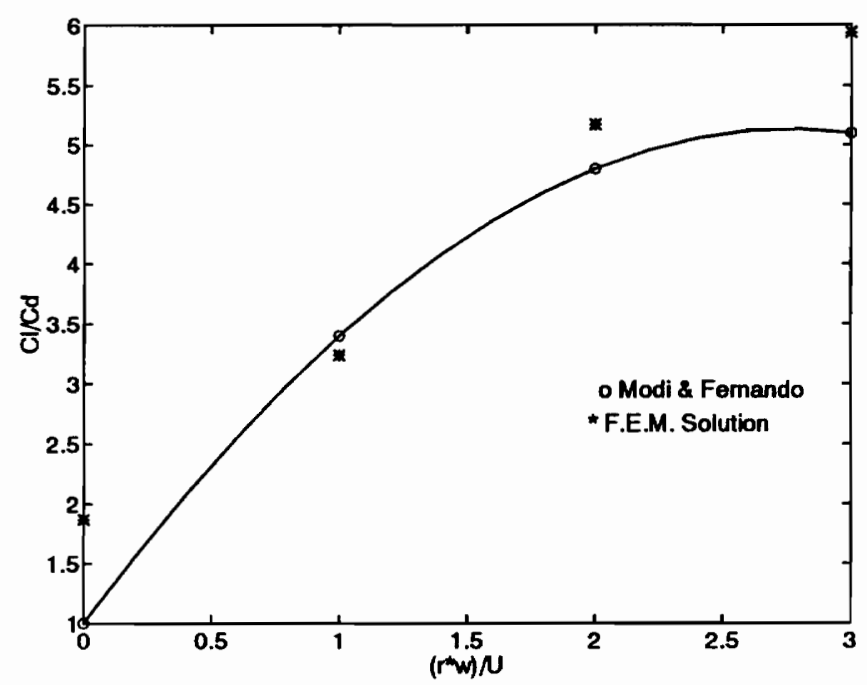

Figure 3.32 Flat plate with Rotating Cylinder at Leading Edge, Comparison with Modi \& Fernando's Flat Plate with rotating L.E. Cylinder and non-rotating T.E. Cylinder

Overall, Modi \& Fernando's ratio between the lift and drag coefficients is smaller than our results. That can be caused by the trailing edge configuration; a bluff body like a cylinder at the trailing edge of the plate is much more likely to produce drag than a wedge shape end. Other influencing factors are the viscosity of the flow and the geometrical dimensions of the plate and cylinder(s). 
When we examine Table 3.10, we conclude that the aerodynamic characteristics of the plate-cylinder system keep improving as the rotational speed of the cylinder increases. This is true, but there exists one consideration that has not been studied in previous experiments: the power associated with the cylinder's rotation. We will see in the next chapter how this component affects the ratio of gain to cost. 


\section{CHAPTER 4}

\section{Optimization Problem}

In this chapter, we formulate an optimal design problem for the aerodynamic performance of a flat-plate airfoil with a leading edge rotating cylinder. But first, it is worthwhile to put our problem in context. Mathematically, a somewhat general class of optimization problems can be stated as:

$$
\begin{array}{cl}
\underset{x \in}{\operatorname{minimize}} & \mathrm{F}(\mathrm{x}) \\
\text { subject to } & \mathrm{c}_{\mathrm{i}}(\mathrm{x})=0, \mathrm{i}=1,2, \ldots, \mathrm{m}^{\prime} ; \\
& \mathrm{c}_{\mathrm{i}}(\mathrm{x}) \geq 0, \quad \mathrm{i}=\mathrm{m}^{\prime}+1, \ldots, \mathrm{m} .
\end{array}
$$

where $x$ represents the problem parameters and $F(x)$, is the objective function. This function provides a measure of the quantity to be minimized. The role of the constraint functions $c_{i}(x)$ is to apply some restrictions on the parameters' values. Both objective and constraint functions are real-valued scalar functions. In this setting, we must describe the parameter vector $x$, the cost function, $F$ and any constraint function, $c$. Before doing so, we describe a non-dimensional formulation of the flow problem.

\subsection{Parameters}

The first step of an optimization problem definition consists in the derivation of the possible system parameters or independent variables. Dimensional analysis can help establish the relative role of the different quantities involved in the flow problem. The Buckingham Pi theorem states that if an equation is dimensionally homogeneous, it can be reduced to a relationship among a complete set of dimensionless products. Using the Buckingham method 24 we can find which dimensionless parameters affects the aerodynamic characteristics of the system. The number of dimensionless groups $i$ is the difference between the number of characteristic quantities $n$ involved in the problem and the number of fundamental dimensions $j$ necessary to express the dimensions on those quantities. Figure 4.1 shows the characteristic quantities of the flat plate with a leading edge rotating cylinder problem. 


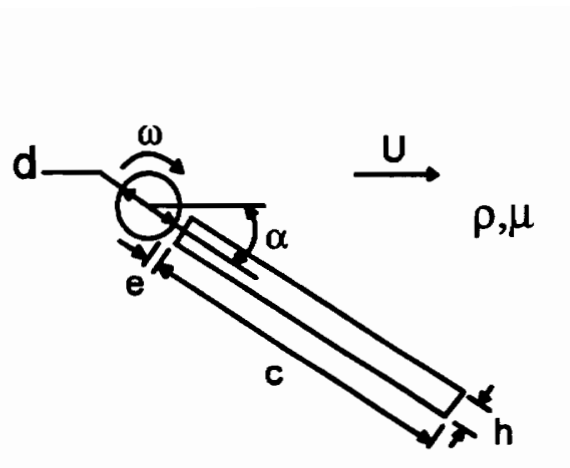

Quantities

$$
\text { F (force) }
$$

$\rho$ (density)

$U$ (free stream velocity)

$c$ (chord)

$\mu$ (viscosity)

$d$ (cylinder diameter)

e (cylinder gap)

$h$ (plate width)

$\alpha$ (angle of attack)

$\omega$ (angular velocity)
Dimensions

$\mathrm{mL} / \mathrm{t}^{2}$

$\mathrm{m} / \mathrm{L}^{3}$

$\mathrm{L} / \mathrm{t}$

$\mathrm{L}$

$\mathrm{m} / \mathrm{Lt}$

$\mathrm{L}$

L

L

none

$\mathbf{t}^{-1}$

Figure 4.1 Characteristic Quantities involved in the Flat Plate with Rotating Cylinder at Leading Edge problem

Quantities: $n=10$ Dimensions: $\mathrm{j}=3$ Dimensionless groups: $\mathrm{i}=\mathrm{n}-\mathrm{j}=7$

Selecting $\rho, U$, and $c$ as the three quantities which, between them, contain all the fundamental dimensions, the dimensionless groups are

$$
\begin{aligned}
& \Pi_{1}=\rho^{21} U^{b 1} c^{c 1} \mu \\
& \Pi_{2}=\rho^{22} U^{b 2} c^{c 2} d \\
& \Pi_{3}=\rho^{23} U^{b 3} c^{c 3} e \\
& \Pi_{4}=\rho^{24} U^{b 4} c^{c 4} h \\
& \Pi_{5}=\rho^{25} U^{b 5} c^{c 5} \alpha \\
& \Pi_{6}=\rho^{26} U^{b 6} c^{c 6} \omega \\
& \Pi_{7}=\rho^{27} U^{b 7} c^{c 7} F
\end{aligned}
$$

The $a j, b j$, and $c j$ are such that the right hand side of these equations is dimensionless.

$$
\left(\frac{m}{L^{3}}\right)^{a 1}\left(\frac{L}{t}\right)^{b 1}(L)^{c 1}\left(\frac{m}{L t}\right)
$$




$$
\begin{aligned}
& a 1+1=0 \\
& -b 1-1=0 \\
& -3 a 1+b 1+c 1-1=0
\end{aligned}
$$

$$
\begin{gathered}
a 1=-1 \\
\Rightarrow \quad b 1=-1 \\
c 1=-1 \\
\Rightarrow \Pi_{1}=\frac{\mu}{c U \rho}=\frac{1}{R e}
\end{gathered}
$$

(2)

$$
\begin{aligned}
& \left(\frac{m}{L^{3}}\right)^{a 2}\left(\frac{L}{t}\right)^{b 2}(L)^{c 2} L \\
& a 2=0 \\
& -3 a 2+b 2+c 2+1=0 \\
& -b 2=0
\end{aligned}
$$

$$
\begin{array}{rlrl}
a 2 & =0 \\
\Rightarrow \quad & b 2 & =0 \\
c 2 & =-1 \\
\Rightarrow & \Pi_{2} & =\frac{d}{c}
\end{array}
$$

Similarly, for (3) and (4), we get

(5) $\alpha$ is already dimensionless

$$
\begin{array}{ll}
\Rightarrow & \Pi_{3}=\frac{e}{c} \\
\Rightarrow & \Pi_{4}=\frac{h}{c} \\
\Rightarrow & \Pi_{5}=\alpha
\end{array}
$$

(6)

$$
\begin{array}{lll}
\left(\frac{m}{L^{3}}\right)^{a 6}\left(\frac{L}{t}\right)^{b 6}(L)^{c 6} \frac{1}{t} & & \\
a 6=0 & \Rightarrow 6=0 \\
-3 a 6+b 6+c 6=0 & b 6=-1 \\
-b 6-1=0 & c 6=1 \\
& \Rightarrow \quad \Pi_{6}=\frac{\omega c}{U} \times \frac{d}{c}=\frac{\omega d}{U} \text { or } \frac{\omega r}{U}
\end{array}
$$




$$
\begin{aligned}
& \left(\frac{m}{L^{3}}\right)^{a 7}\left(\frac{L}{t}\right)^{b 7}(L)^{c 7} \frac{m}{L t^{2}} \\
& \text { a7 }+1=0 \quad \text { a7 }=-1 \\
& -3 a 7+b 7+c 7+1=0 \quad \Rightarrow \quad b 7=-2 \\
& -b 7-2=0 \quad c 7=-2 \\
& \Rightarrow \quad \Pi_{7}=\frac{F}{\rho U^{2} c^{2}} \times 2=C_{L}, C_{D} \text { when } F=L, D
\end{aligned}
$$

Finally, we can express the dimensionless lift and drag coefficient as a function of the other dimensionless groups:

$$
C_{L}, C_{D}=f\left(R e, \alpha, \frac{U}{\omega r}, \frac{d}{c}, \frac{h}{c}, \frac{e}{c}\right)
$$

In this study, we set fixed values for $R e, d, h$ and $e$. For the sake of simplicity, we choose only one optimization parameter: the most influent parameter acting on the boundary layer separation delay, that is the ratio of the free stream velocity to the tangential speed of the cylinder $\mathrm{U} / \omega \mathrm{r}$. It is also the most straight forward choice for the finite-element procedure since, unlike any geometrical parameter, it does not require any changes to the grid. The geometrical dimensions for the flat plate are based on previous models ${ }^{14,28}$. The Reynolds number is fixed to a moderate value for numerical considerations. At high Reynolds number, the flow quickly becomes unsteady as we increase $\alpha$ and it is not possible to obtain convergence for the steady finite element problem. At very low Reynolds number, the influenced zones are bigger and the boundary-layer effect of viscosity is more widespread, since the flow is more uniform over the domain. This is quite undesirable, considering that the purpose of this study is to analyze the boundary-layer behavior. By fixing the Reynolds number to a value of a thousand, we set a compromise between those two cases. The angle of attack $\alpha$ will be varying from zero to the airfoil stall value, each angle providing a different flow problem. In this sense, it can be considered more as an independent parameter. An value of cylinder optimum rotating speed is found for each angle of attack. 


\subsection{Objective function}

The objective function serves the goal of describing good performance. In the present problem, there are two important physical quantities:

1. the lift produced by the airfoil (L)

2. the power required to move the airfoil $(\mathrm{P})$

The power $\mathrm{P}$ consist of two contributions:

1. the power to translate the airfoil $(\mathrm{drag} \cdot \mathrm{U})$

2. the power to rotate the cylinder $\left(\mathrm{P}_{\mathrm{cy}}\right)$

The formulation of the objective function depends on the type of minimization problem we define. Using the general problem formulation (4.1), we can generate three particular formulations for our problem:

\section{Problem I}

$$
\text { minimize }-\mathrm{L}(\omega) \text { or } \mathrm{L}^{-1}(\omega)
$$

$$
\omega \in \mathfrak{a}
$$

\section{Problem II}

$$
\text { subject to } c(\omega)=P-C_{p}=0
$$

$$
\text { minimize } \quad P(\omega)
$$

$$
\omega \in \mathbb{R}
$$

$$
\text { subject to } c(\omega)=L-C_{L}=0
$$

\section{Problem III}

$$
\begin{aligned}
& \underset{\omega \in \mathbb{R}}{\operatorname{minimize}} \mathrm{P}(\omega) / \mathrm{L}(\omega) \\
& \text { subject to no constraint }
\end{aligned}
$$

The necessary conditions for constrained minimization are stated as a vanishing derivative of the Lagrangian ${ }^{23}$. For an unconstrained problem, the necessary condition is simply the zero derivative of the objective function itself. 
Consider the necessary conditions for the previous problems:

I.

$$
\begin{gathered}
L^{\mathrm{I}}\left(\omega, \lambda^{\mathrm{I}}\right) \equiv-L(\omega)+\lambda^{\mathrm{I}}\left(P(\omega)-C_{P}\right) \\
-L^{\prime}+\lambda^{\mathrm{I}} P^{\prime}=0 \\
P-C_{P}=0 \\
\\
\mathcal{L}^{\mathrm{II}}\left(\omega, \lambda^{\mathrm{II}}\right) \equiv P(\omega)+\lambda^{\mathrm{II}}\left(L(\omega)-C_{L}\right) \\
P^{\prime}+\lambda^{\mathrm{II}} L^{\prime}=0 \\
L-C_{L}=0
\end{gathered}
$$$$
\text { II. } \quad P^{\prime}+\lambda^{\mathrm{Il}} L^{\prime}=0
$$

$$
\text { III. } \frac{P^{\prime}}{L}-\frac{P}{L^{2}} L^{\prime}=0 \text { or } P^{\prime}-\frac{P}{L} L^{\prime}=0
$$

We can show that problems I and II are just two different formulations for the same problem. Suppose $\omega^{*}$ solves ("solving" here means that $\omega^{*}$ satisfies the first order necessary conditions, technically we say that $\omega^{*}$ is a stationary point for the minimization problem) I for the specified $C_{p}$, i.e. $\exists \lambda^{I *}$ so that

$$
-L^{\prime}\left(\omega^{*}\right)+\lambda^{1} P\left(\omega^{*}\right)=0
$$

and

$$
P\left(\omega^{*}\right)=C_{P},
$$

then $\omega^{*}$ "solves" II for $C_{L}=L\left(\omega^{*}\right)$ if we replace

$$
\lambda^{\mathrm{II} *}=1 / \lambda^{\mathrm{I}} \text { * }
$$

in expression (4.14). The argument can be reversed to show that if $\omega^{*}$ "solves" II for a specified $C_{L}$ then it also "solves" I.

Problem III can be considered as a particular case of Problems I and II. Suppose that $\hat{\omega}$ solves Problem III and that $L(\hat{\omega})=\hat{C}_{L} \neq 0$. We claim that $\hat{\omega}$ solves Problem II (for the specified value $\left.C_{L}=\hat{C}_{L}\right)$. To demonstrate, we observe that the choice $\lambda^{I I}=-P(\hat{\omega}) / L(\hat{\omega})$ leads to a solution of the system (4.14). Because no constraint functions are involved, this problem is simpler that the other two and will be the chosen one to formulate the optimization problem. The objective function is therefore established as the ratio of 
power over lift, or if we tranform the problem as a maximization, the ratio of lift over total power:

$$
F(\omega)=L \cdot U /\left(D \cdot U+P_{c y l}\right)
$$

To simplify the notation, we will refer to $F(\omega)$ as $L / P$ since the free stream velocity $U$ is equal to one. The "gain" here translates as the lift $L$ acting on the system airfoil-cylinder multiplied by the free stream velocity $U$. The "cost" includes the product of the $\operatorname{drag} D$ and the free stream velocity, as well as the power $P_{\text {oyl }}$ required to rotate the cylinder at an angular speed of $\omega$.

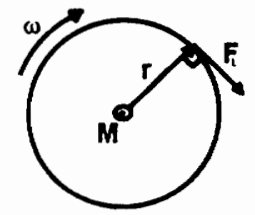

The power necessary to rotate the cylinder is computed as the product of the moment $M$ operating on the cylinder and its rotating speed $\omega$. This moment is defined as the cross product of the force acting on the edge of the cylinder and its lever arm, the radius vector. Theoretically, the normal component of the force brings no contribution to the moment since its

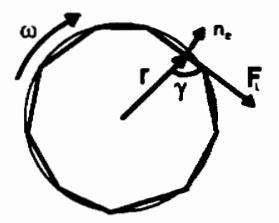
cross product with the radius vector is zero. Now, in a finite element approximation, the boundary defined by the elements does not coincide with a perfect circle. The angle between the position vector and the edge

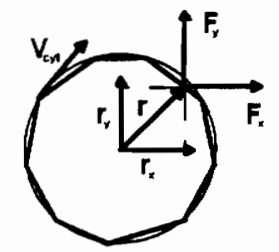

Figure 4.2

Rotation Power of the element, $\gamma$, differs from $90^{\circ}$. Therefore, the cross product between the normal force component and the radius vector cannot be excluded from the moment calculation. The use of a Cartesian decomposition of the force and the position vectors takes this consideration into account. The equation for the power calculation is thus formulated as:

$$
\begin{aligned}
P_{\text {cyl }} & =\left[\left(F_{x} \hat{i}+F_{y} \hat{j}\right) \times\left(r_{x} \hat{i}+r_{y} \hat{j}\right)\right]_{\text {cyl }} \omega \\
& =\left[F_{x} r_{y}-F_{y} r_{x}\right]_{c y}\left(\frac{V_{c y}}{|r|}\right) \\
& =\int_{\Gamma_{\text {cyy }}}\left[\left(\tau_{x x} n_{x}+\tau_{x y} n_{y}\right) r_{y}-\left(\tau_{x y} n_{x}+\tau_{y y} n_{y}\right) r_{x}\right]_{c y 1} d \Gamma_{\text {cyl }}\left(\frac{V_{\text {oy }}}{|r|}\right)
\end{aligned}
$$


Note that, naturally, this integral is only performed along the cylinder contour. The expression for the stress tensor and the integration procedure are given in section 2.5 of chapter 2 .

\subsection{Problem definition}

With the objective function and parameters defined, we now possess the necessary tools to construct the optimization problem. Since we choose only one parameter for the objective function, we restrict our choice to methods for univariate functions. In the previous section we defined the optimization problem as an unconstrained case. There is no constraint applied on lift or power but some restrictions may be imposed on the parameter $\omega$. Consequently, we must define a feasible region (interval, in a onedimensional case) in which lies all the feasible points $\left(\omega^{*}\right)$ to the optimization problem. In order to be optimal, a point must be feasible and respond to certain qualifications called optimality conditions. In a one-dimensional case, the only restriction that may be applied to the single parameter (here, the cylinder rotational speed $\omega$ ) is simple bounds on its value. The feasible interval for the cylinder rotational speed is described by:

$$
0.0<\omega^{\star}<4.0
$$

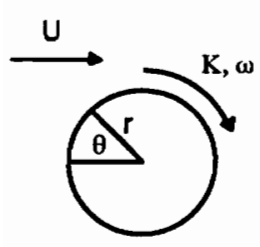

The lower bound restricts the cylinder to a clockwise rotation. The upper bound comes from circulation theory. In a flow past a rotating cylinder of radius $r$ superimposed on a uniform flow with velocity $U$, if the tangential velocity of the cylinder is four times the free stream velocity, the circulation $\mathrm{K}$ is equal to $4 \pi \mathrm{r} U$. The velocity at the surface is equal to $2 U \sin \theta+\mathrm{K} /(2 \pi r)$. Then, there is only one point of stagnation, at $\theta=3 \pi / 2$ and the velocity is everywhere in the same direction as that of the surface. As the cylinder rotational speed is increased, the stagnation point leaves the surface and moves downward. Therefore, at $(r * \omega) / U \geq 4$, there is no further tendency of the circulation to expands. Consequently, since the lift is function of the circulation, it will remain the same as $\omega$ increases. Considering that the power required to rotate the cylinder increases monotonically with $\omega$, it follows that the same gain would be generated at a higher cost. This is a theoretical result based on potential flow. In practice, some circulation is still generated by the cylinder at a tangential velocity higher that $4 \mathrm{U}$. However, the lift 
produced is small compared to the associated drag, therefore there is no advantage to go beyond this value.

For the unconstrained univariate case, the necessary conditions for an interior maximum are:

$$
\begin{aligned}
& F^{\prime}\left(\omega^{*}\right)=0 \\
& F^{\prime \prime}\left(\omega^{*}\right) \leq 0 .
\end{aligned}
$$

The objective function, as it is described in section 4.2, gives one value of $(\mathrm{L} / \mathrm{P})$ for a single input of $\omega$. To obtain a description of the objective function over the feasible interval, we must compute a finite element solution and generate from it a data point for the objective function at various values of $\omega$ over the interval.

\subsection{Methodology}

One optimization problem must be solved for each angle of attack. The mesh used to discretize the model at an angle $\alpha$ is fixed for the whole process. The first step is therefore to obtain a grid refined to a reasonable accuracy for a low rotational speed, as described in section 3.2. Subsequently, we vary the boundary conditions on the cylinder (i.e. $\omega$ ) with a fixed increment until the upper bound limit is reached. At each iteration, we compute the finite element solution at the given rotational speed and generate from it a data point for the objective function. Each solution uses the one previously obtained as initial conditions. This constitutes the first set of data points.

Of the three methods generally used for univariate minimization (Fibonacci search, Golden section search and Polynomial interpolation)23, we choose the polynomial interpolation method. When the function to optimize is smooth, this method provides an easy and efficient way to locate the optimum. The objective function is approximated by a low-degree polynomial, say 2 or 3 , whose minimum (or maximum) is easily found. We first pass a least squares approximation of a third order polynomial (in the case where we would have only three or four initial data points, we apply a Lagrange second or third order polynomial) through all data points. The maximum of the cubic polynomial provides a first estimate for the parameter's optimal value.

Once we have located the initial guess for the maximum, we use the four surrounding data points and work with an exact third order polynomial to iterate. When a new 
estimate for the maximum is calculated, we compute a new finite-element data point with the rotational speed corresponding to the polynomial estimate. If the difference between the new data point and the polynomial estimate is larger that the convergence criteria, we perform a new iteration. Since we have now five points, we must eliminate one of them in order to obtain an exact third order polynomial for the next iteration. We will describe the elimination process in the next section. The iterative process goes on until the relative error between the polynomial estimate and the finite element solution reaches a value below the desired tolerance. If there is no maximum in the feasible interval, the larger value between the lower and the upper bound function values is taken as the optimum.

\subsection{Application}

To understand how the optimization process is applied, we will study in this section one case at a fixed angle of attack $\left(\alpha=20^{\circ}\right)$. Before starting with the optimization process, we elaborate a set of data points for the objective function (4.19). For lower angles of attack (from $0^{\circ}$ to $15^{\circ}$ ) we could obtain a solution for the flow problem with zero rotation of the cylinder and therefore generate data points for the whole feasible interval.

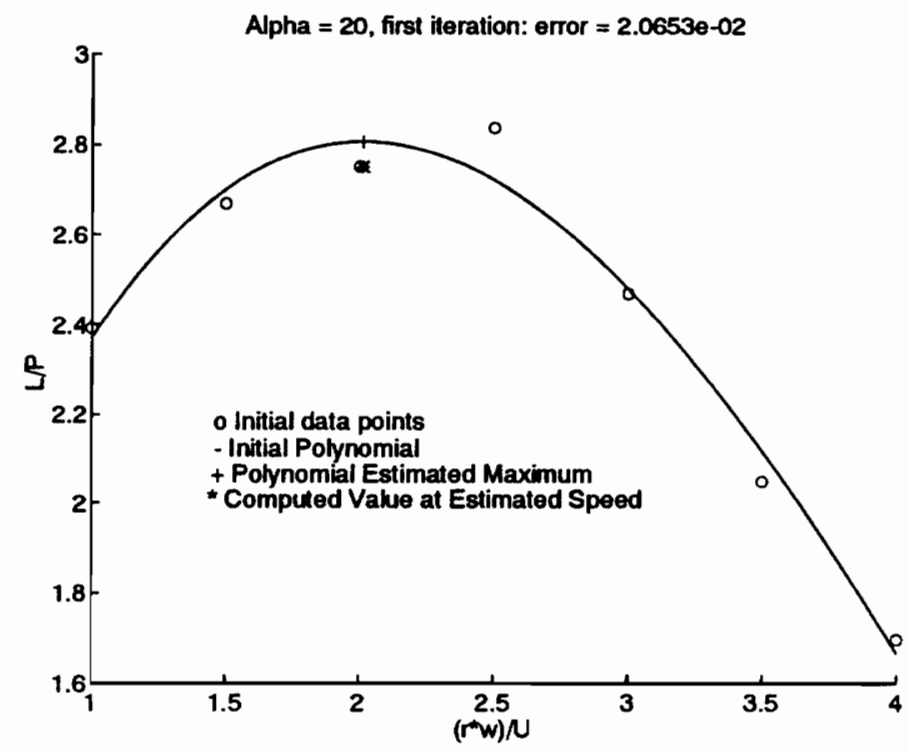

Figure 4.3 Initial Curve for the $20^{\circ}$ Cylinder-Flat Plate System

However, when we reach the $20^{\circ}$ case, separation occurs if the cylinder is not rotating and we need to increase its rotational speed until the flow is reattached to be able to get a 
finite element solution (see discussion in paragraph 3.2.2). Figure 4.3 shows the initial curve for the optimization study.

Starting the optimization process, we located an initial estimate for the maxima at $(r \cdot \omega) / U=2.2$. The optimization convergence criteria is fixed at 1.0e-04 of relative error. Since the new data point generated from this rotational speed was too far from the estimate (relative error $=2.0653 \mathrm{e}-02$ ), we kept the four surrounding data points and passed through them an exact third order polynomial. With this polynomial, a new estimate for the maximum was calculated and compared to the f.e.m. solution computed with the corresponding angular speed (Figure $4.4 \mathrm{a}$ )).

Since the difference between the two values was still larger then the desired tolerance, we had to throw out one of the five points for the next iteration. Since we want a maximum, the first idea that comes to mind is to simply keep the four points with largest $(\mathrm{L} / \mathrm{P})$ ratio. However, there exists no guarantee, as we will see, that the maximum is located within the interval selected in that manner. Figure 4.4 and table 4.1 present in details the iterative optimization process.

a) Second Iteration

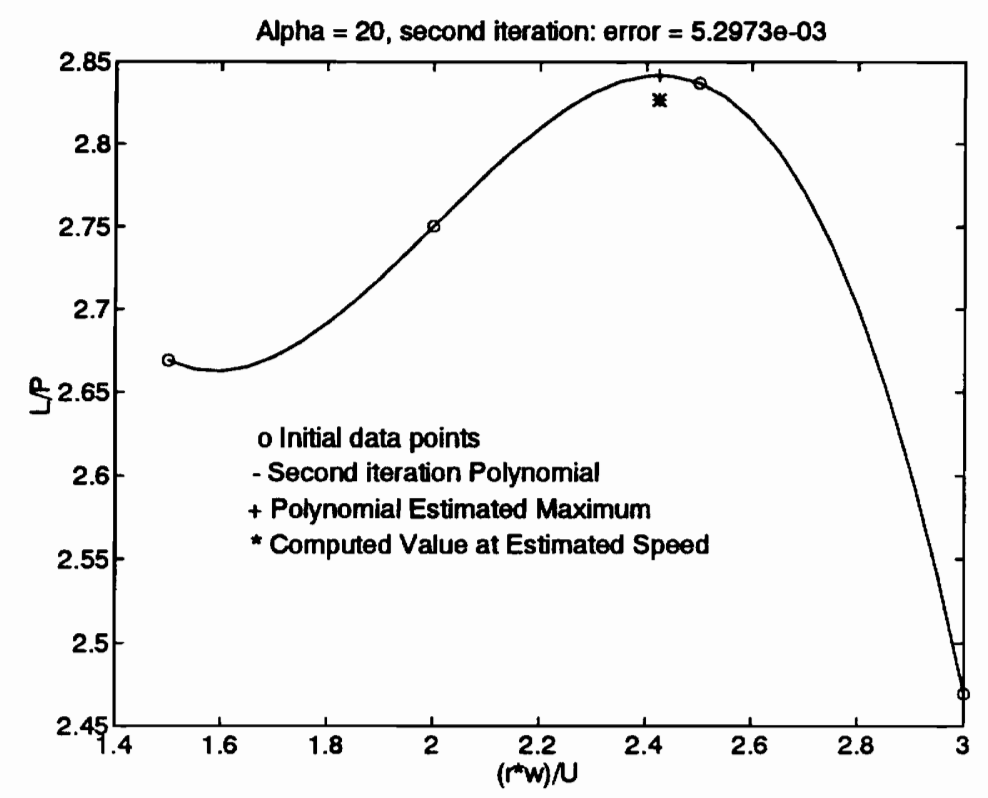

Figure 4.4 Iterative Optimization Process for the $20^{\circ}$ Cylinder-Flat Plate System 
b) Third Iteration

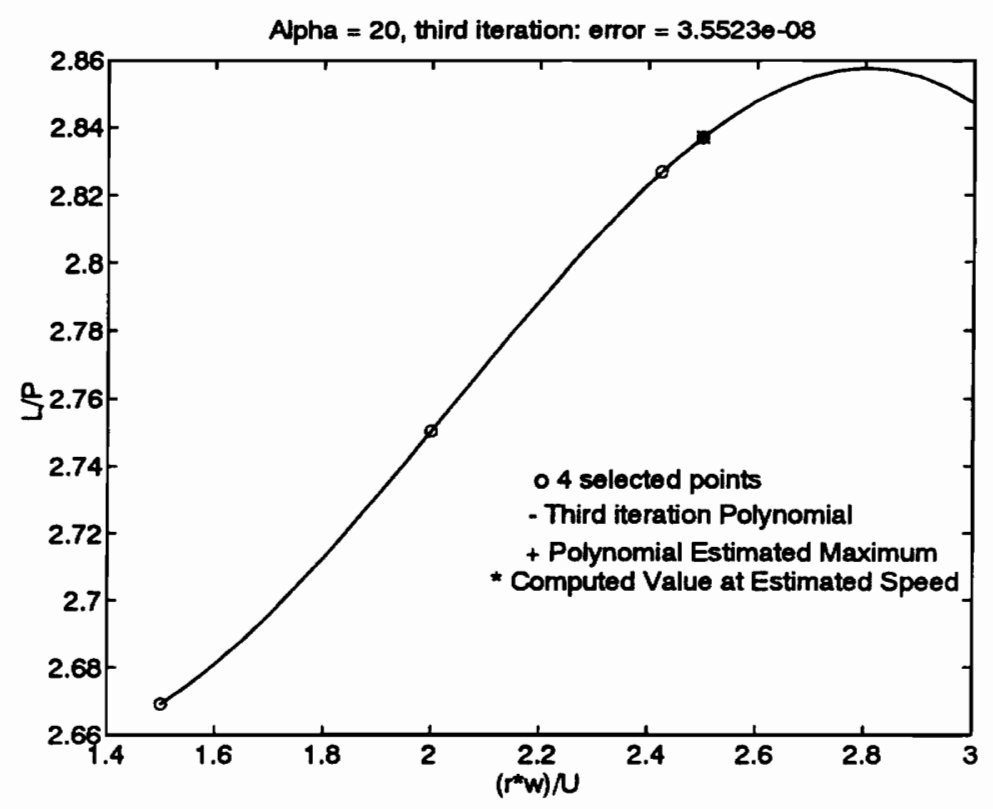

c) Corrected Third Iteration

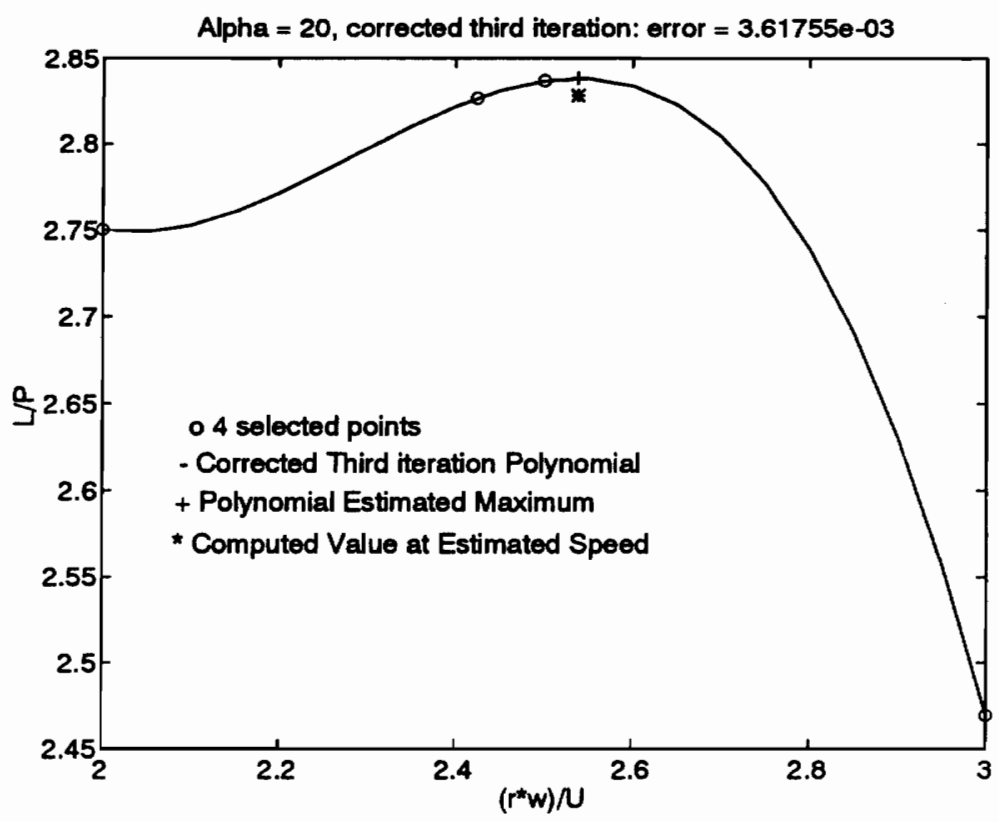

Figure 4.4 Iterative Optimization Process for the $20^{\circ}$ Cylinder-Flat Plate System 
d) Fourth Iteration

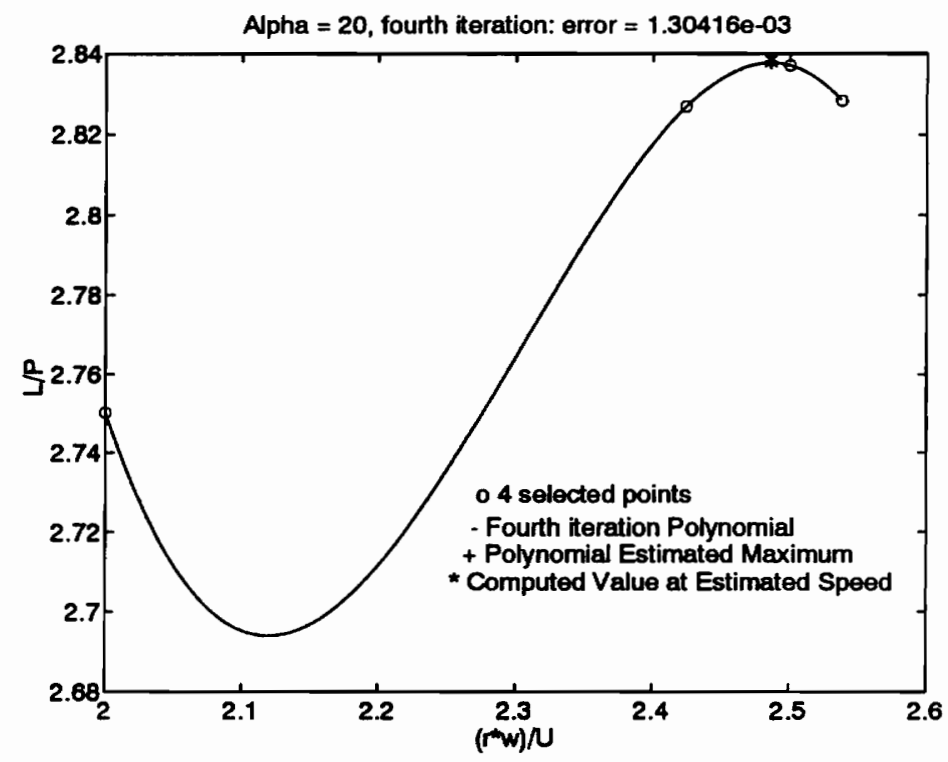

e) Fifth Iteration

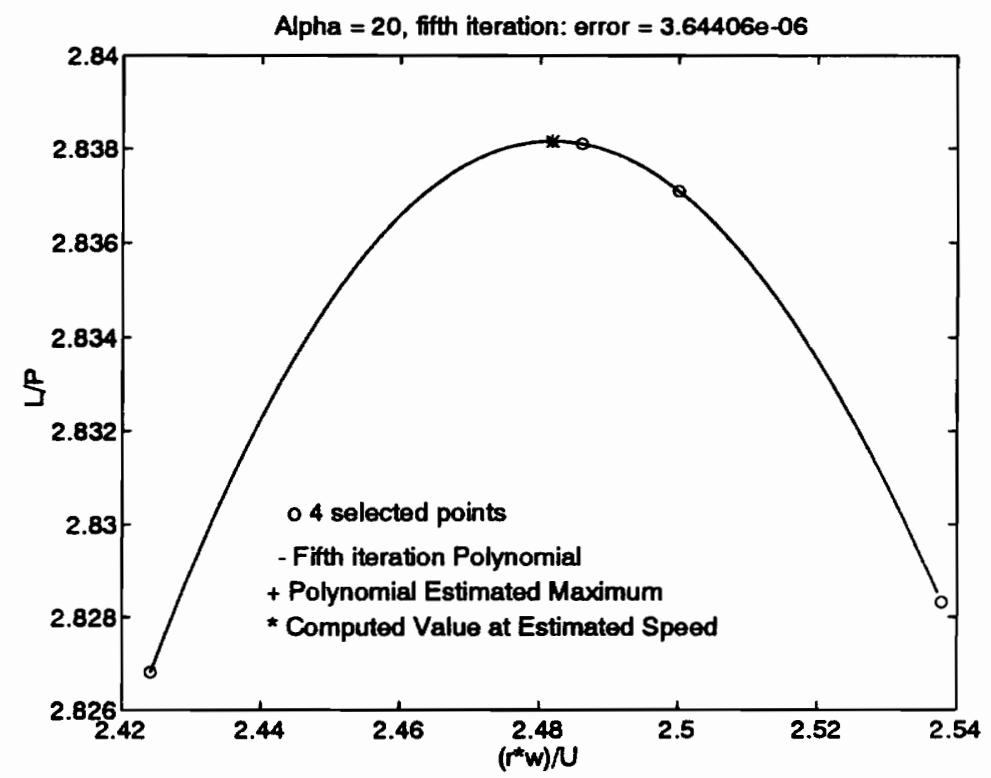

Figure 4.4 Iterative Optimization Process for the $20^{\circ}$ Cylinder-Flat Plate System 
Table 4.1 Iterative Optimization Process for the $20^{\circ}$ angle case

\begin{tabular}{|c|c|c|c|c|}
\hline Iteration & $\begin{array}{c}\text { Estimated } \\
\text { Optimal } \\
\text { Speed }\end{array}$ & $\begin{array}{c}\text { Estimated } \\
\text { Optimum } \\
\text { L/P }\end{array}$ & $\begin{array}{c}\text { Computed } \\
\text { F.E.M. } \\
\text { Optimum }\end{array}$ & $\begin{array}{c}\text { Relative } \\
\text { Error }\end{array}$ \\
\hline \hline 1 & 2.01194 & 2.80676 & 2.74996 & $2.065 \mathrm{e}-02$ \\
\hline 2 & 2.42416 & 2.84178 & 2.82681 & $5.297 \mathrm{e}-03$ \\
\hline 3 & 2.50000 & 2.83709 & 2.83709 & $3.552 \mathrm{e}-08$ \\
\hline $3 \mathrm{r}$ & 2.53780 & 2.83854 & 2.82831 & $3.618 \mathrm{e}-03$ \\
\hline 4 & 2.48604 & 2.83773 & 2.83810 & $1.304 \mathrm{e}-04$ \\
\hline 5 & 2.48180 & 2.83816 & 2.83815 & $3.644 \mathrm{e}-06$ \\
\hline
\end{tabular}

If we keep the four largest values in this case, we notice in the enlarged scale of Figure 4.4 b) that the polynomial maxima is located outside the interval delimited by the four selected points. Now, because no maximum was found within the interval, the largest function value of the delimiting points would be picked as the final optimum. Considering Figure 4.3 , nothing indicates for sure that the maximum is really 2.50000 to a tolerance below $1.0 \mathrm{e}-04$ ! To avoid this error, we need a more reliable algorithm for the choice of the four "better" points to keep.

One algorithm that could be considered is an analysis of the difference in the function value among the five given points. However, this may be too elaborate for a problem of this size. Analyzing the problem more closely, we realize that if an optimum has already been detected in a previous iteration, it must exists within the five selected points. An easy solution to the problem presented above is to simply select a different set of four points by throwing out the second smallest point instead of the one previously chosen. There is little chance that the same error will occur twice within such a small number of points. In the case that it did, we just stop the process and generate a message that the algorithm has failed. At that point, one could look at the curves involved in the optimization process and see easily the cause of the problem. Figure $4.4 \mathrm{c}$ ) shows the corrected polynomial approximation. As suspected, the maximum is found within the second interval selected within the five points of iteration 2 . Finally, two more iterations were necessary to obtain the desired accuracy (Figure $4.4 \mathrm{~d}$ ) and $\mathrm{e})$ ). 
Applying this algorithm to all angles of attack, we finally obtain the ideal rotational speed trajectory for the flat plate with rotating leading edge cylinder problem. Chapter 5 presents the final results and comments to this study. 


\section{CHAPTER 5}

\section{OPTIMIZATION RESULTS}

In this chapter, we presents the main results on our optimization study for the flat-plate airfoil with leading-edge cylinder. The basic procedure for modelling the flow and for solving the optimization problem have been discussed in earlier chapters.

\subsection{Flat Plate Alone}

To begin the study on the effects of the rotating cylinder on the aerodynamic performance of the flat plate profile, we first look at the results generated without the cylinder. Note that obviously, the power component of the objective function in this case contains only the aerodynamic drag. Figure 5.1 presents the objective function variations with respect to the angle of attack of the plate. No solution could be obtained for angles greater than $15^{\circ}$.

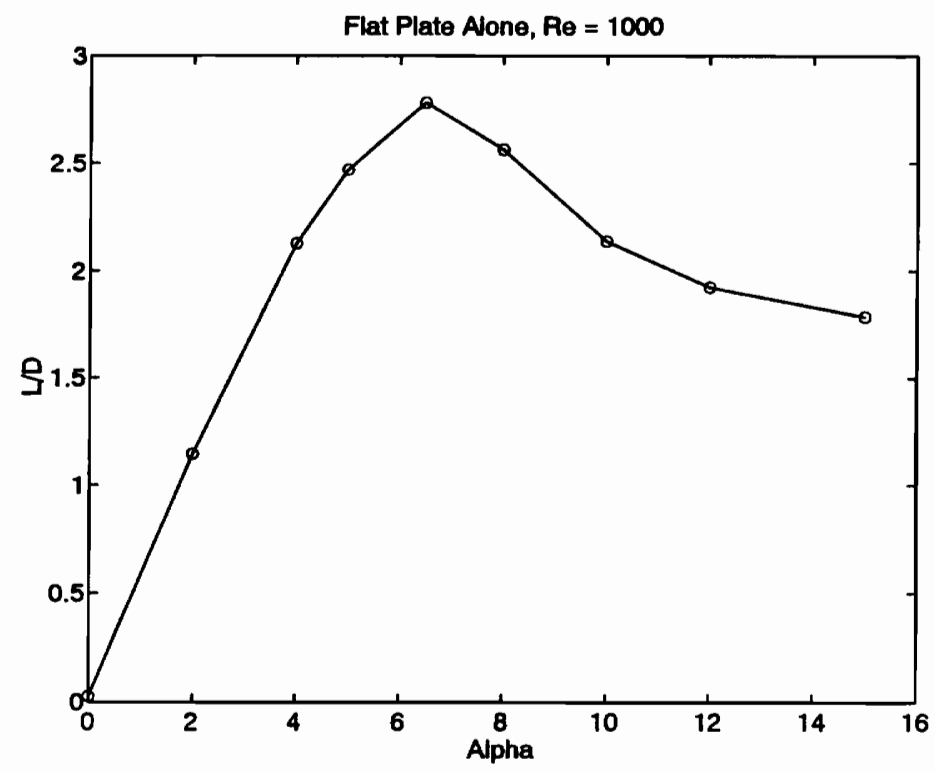

Figure 5.1 Lift to Drag Ratio versus Angle of Attack for Flat Plate Alone

As expected for a flat surface, no lift is generated at zero angle of attack. The lift to drag ratio subsequently reaches a peak around $6.5^{\circ}$ and then decreases as $\alpha$ increases to $15^{\circ}$. This is due to a substantial increase in drag compare to the lift around this same angle, as shown in Figure $5.2 a$ ) and $b$ ). 
a) Lift coefficient

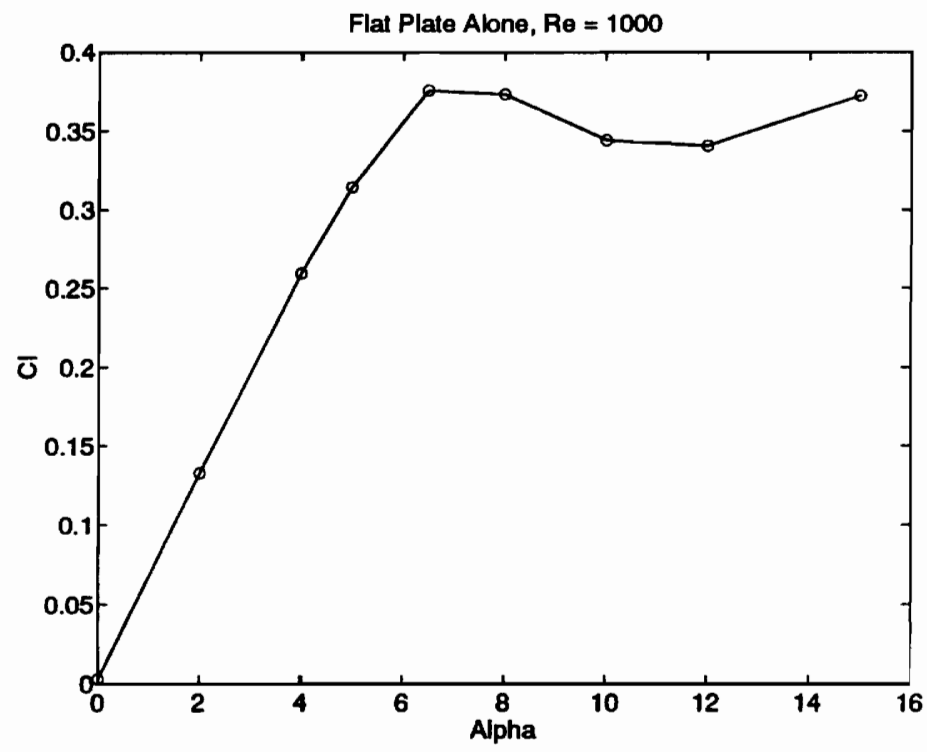

b) Drag Coefficient

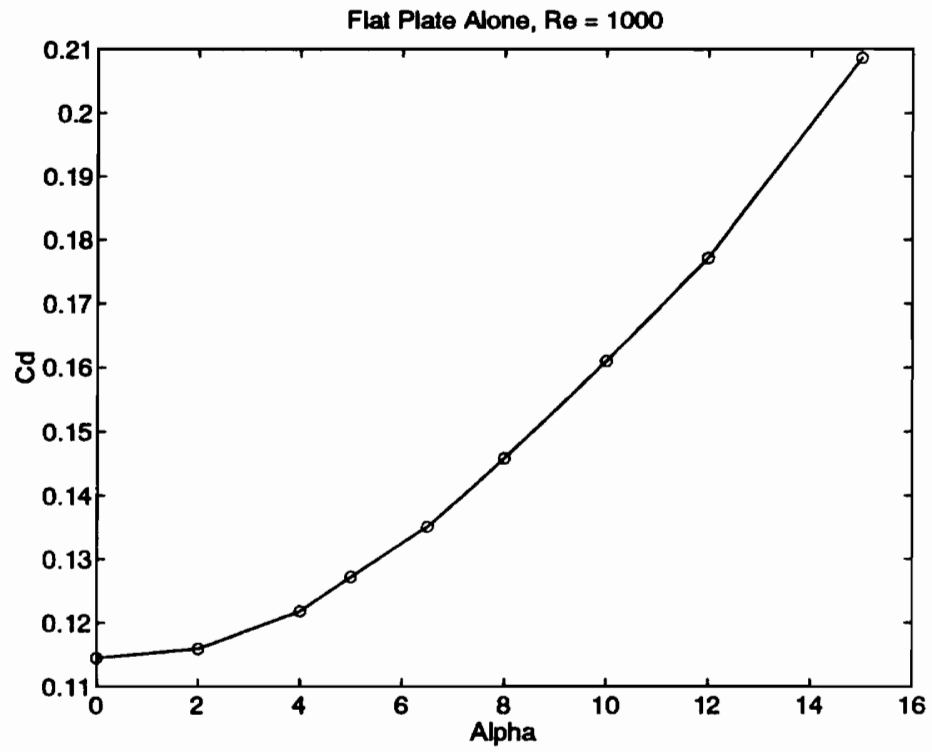

Figure 5.2 Lift and Drag coefficients versus Angle of Attack for Flat Plate Alone 


\subsection{Flat Plate with Rotating Cylinder at Leading Edge}

Before discussing the optimization results, we calibrate the computed aerodynamic forces by comparing them in a qualitative manner to similar experiments. In previous studies on the effects of a rotating cylinder as a boundary-layer control, most consider only the lift coefficient as a performance measure. Modi et al.14 analyzed briefly the lift to drag ratio in their investigation on a NACA 63-218 profile. However, the camber of the model and the difference in flow conditions $\left(\operatorname{Re}=2.9 \times 10^{6}\right)$ are such that there is no basis for comparison. Despite the larger plate thickness and cylinder diameter as well as the trailing edge configuration of Modi \& Fernando's flat plate2s, we can compare qualitatively the lift coefficient versus angle of attack graphs. While it has no major influence on the lift, such difference in thickness and diameter has a substantial effect on the drag so there is no point in this case to compare drag coefficients.
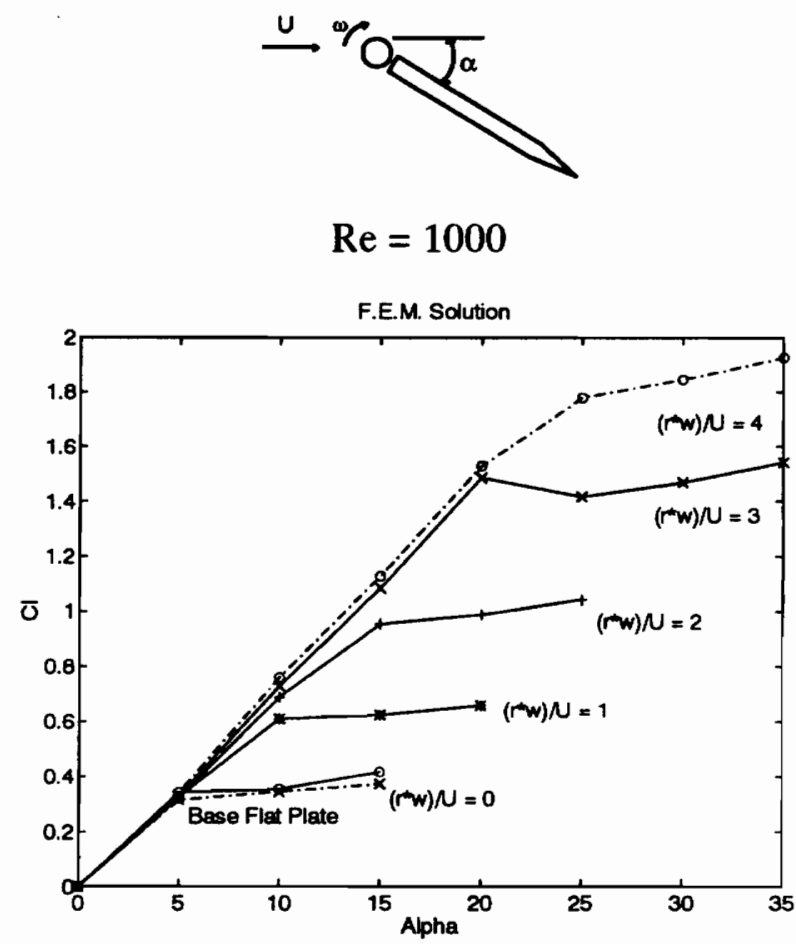

Figure 5.3 Finite Element Model, Lift Coefficient versus Angle of Attack 
a) Modi \& Fernando, Flat Plate with Leading and Trailing Cylinders

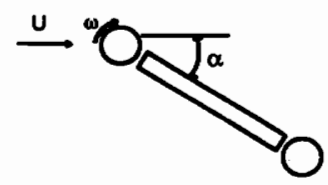

$$
\operatorname{Re}=30000
$$

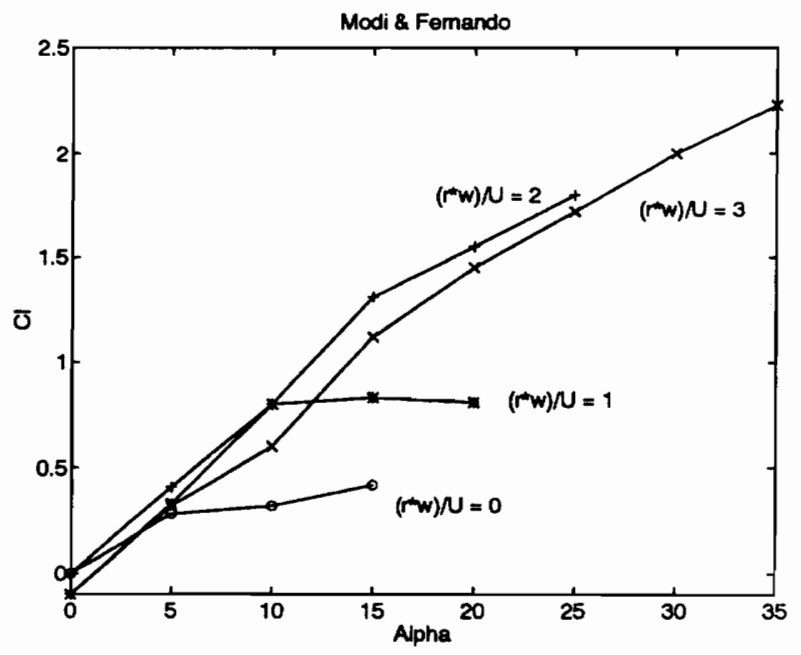

b) Mokhtarian, Modi \& Yokomiso, Joukowsky Airfoil with Leading Edge Cylinder

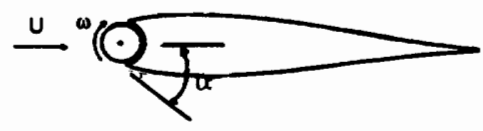

$$
\operatorname{Re}=46200
$$

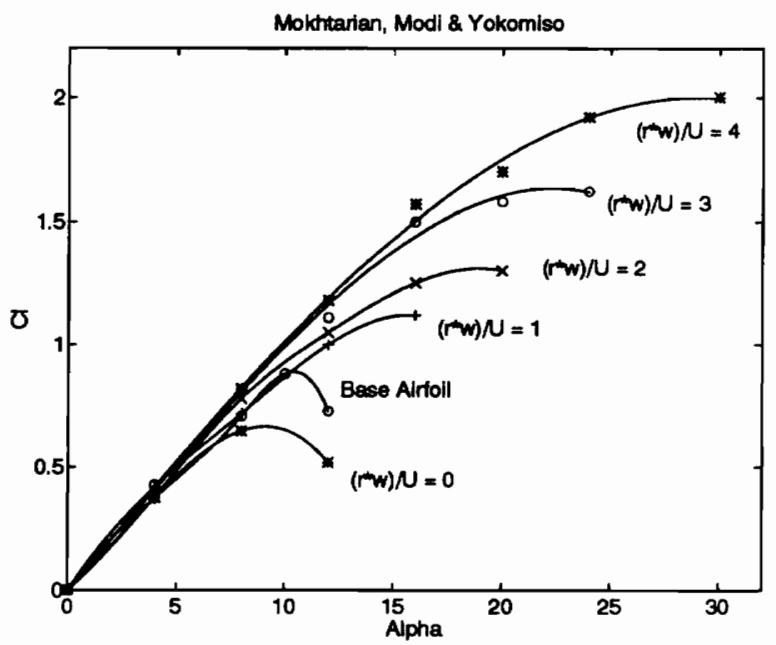

Figure 5.4 Experimental Results, Lift Coefficient versus Angle of Attack 
Figure 5.3 shows the lift coefficient obtained with our finite element model at several angles of attack for various cylinder angular speeds. If we compare this graph with Figure 5.4 a), we observe a fairly good agreement between the lower speed curves $\left(\left(r^{*} w\right) / \mathrm{U}=0\right.$ and $\left.\left(r^{*} w\right) / U=1\right)$. At higher speeds, however, Modi \& Fernando obtained larger lift coefficients and the qualitative agreement between the curves deteriorates.

Considering Figure 5.4 b), we observe that the general trend of the different angular speed curves within this graph is similar to what we observe in the finite element model graph. As the angular speed increases, the lift curve gradually extends without change in slope and the stall peak flattens. Although it is difficult to compare these three diverse cases since the models and the flow conditions present many differences, we can conclude that the finite element model gives reasonable results for the computation of aerodynamic forces.

We are now ready to discuss of a numerical modeling and optimization effort. Recall that our performance index is the ratio of lift produced to power supplied. This is a natural extension of the usual lift-to-drag ratio since we explicitly penalise for the power required to rotate the leading-edge cylinder. Shown in figure 5.5 a) is the classical lift to drag ratio, while figure $5.5 \mathrm{~b}$ ) shows our lift-to-power ratio.

Note in figure 5.5 a) that the inclusion of the drag in the performance ratio increases its scale and induces curvature in the stall peak. If we only consider the translation power (drag) in the cost function, we reach the conclusion that the maximum cylinder angular speed provides the optimum performance for all angles of attack. However, there exists also a cost associated with the rotation of the cylinder which is taken into account in figure $5.4 \mathrm{~b}$ ). The geometry of the different speed ratio curves is drastically changed by this inclusion. The maximum performance ratio at highest angular speed (4) is reduced approximately by $70 \%$ and this reduction decreases gradually to $20 \%$ for lowest angular speed (1). Figure 5.6 shows the lift to total power ratio for intermediate angular speeds to the ones presented in figure $5.5 \mathrm{~b}$ ). Considering the intricate curves of figure 5.6, we see that the optimum value for the cylinder angular speed is not easily determined just by looking at this graph. The optimization procedure described in chapter 4 provides the necessary tool to determine the rotational speed that will generate the optimal performance ratio. Figures 5.7 through 5.15 present the optimization problems for all angles of attack. 
a) Lift to Drag ratio

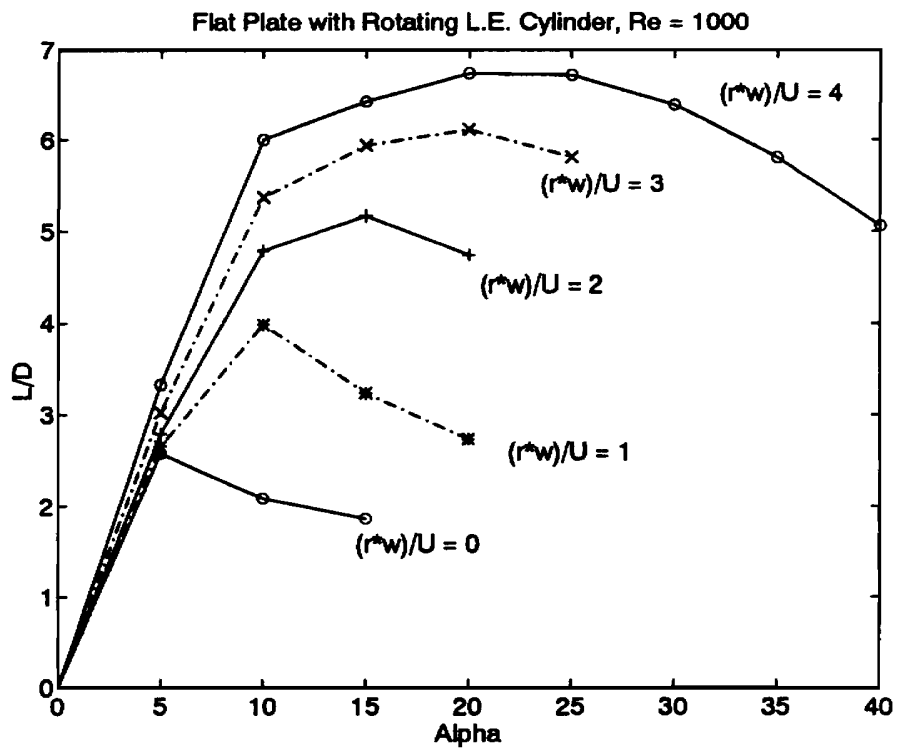

b) Lift to Total Power ratio

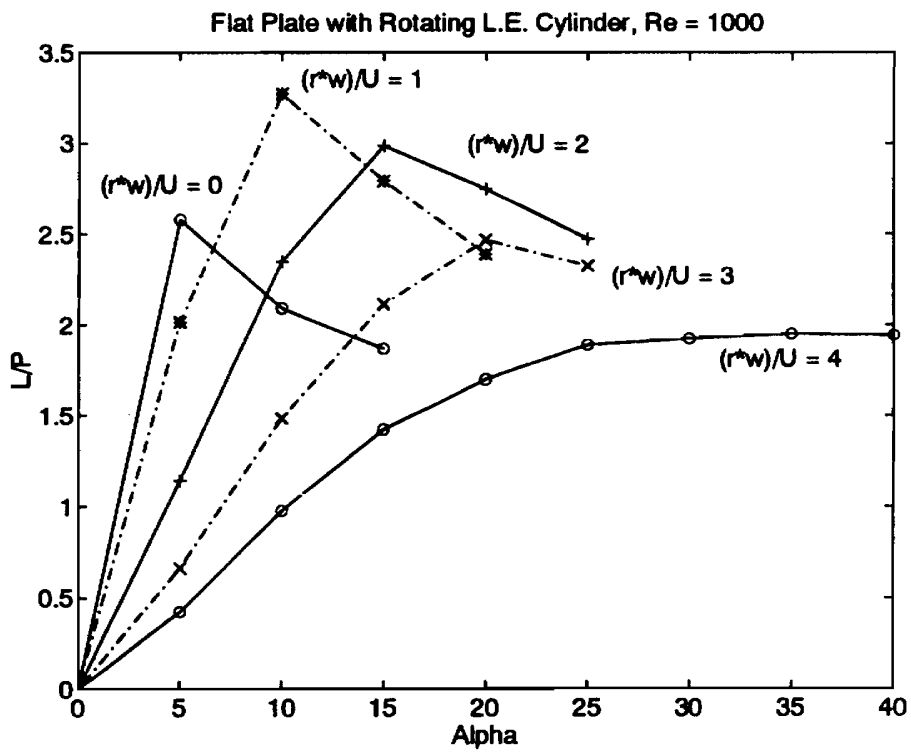

Figure 5.5 Effect of Including Rotation Power in the Performance Ratio 
a) $0.0<(r \cdot \omega) / U<2.0$

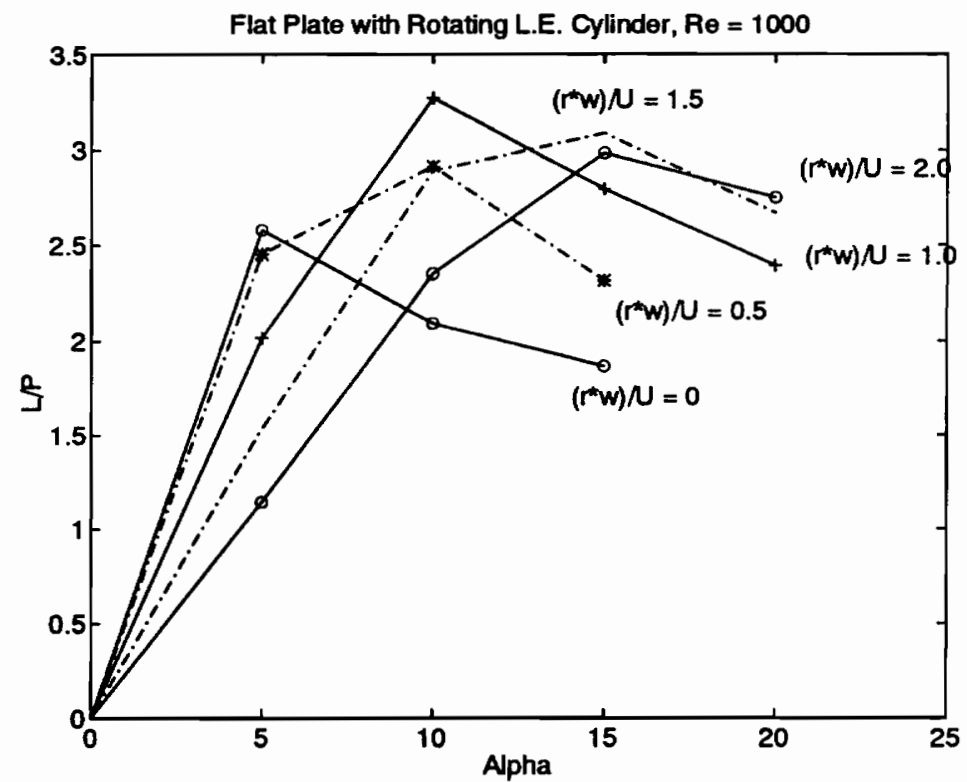

b) $2.0<(r \cdot \omega) / U<4.0$

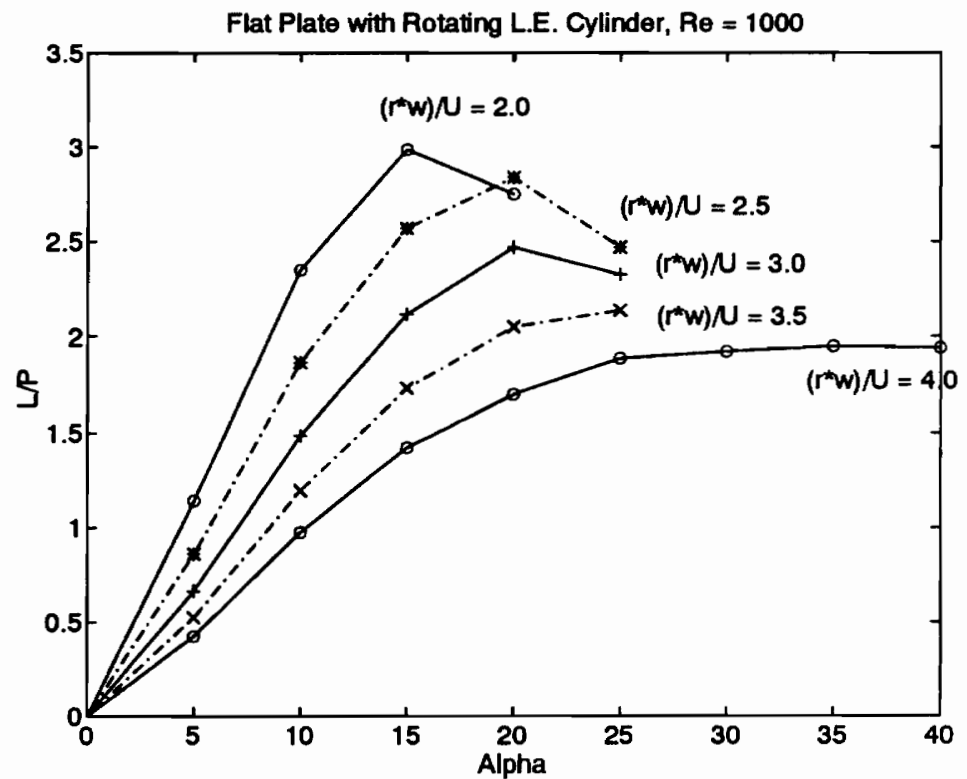

Figure 5.6 Lift to Total Power ratio for various Rotation Speeds 
a) Non Symmetrical Domain

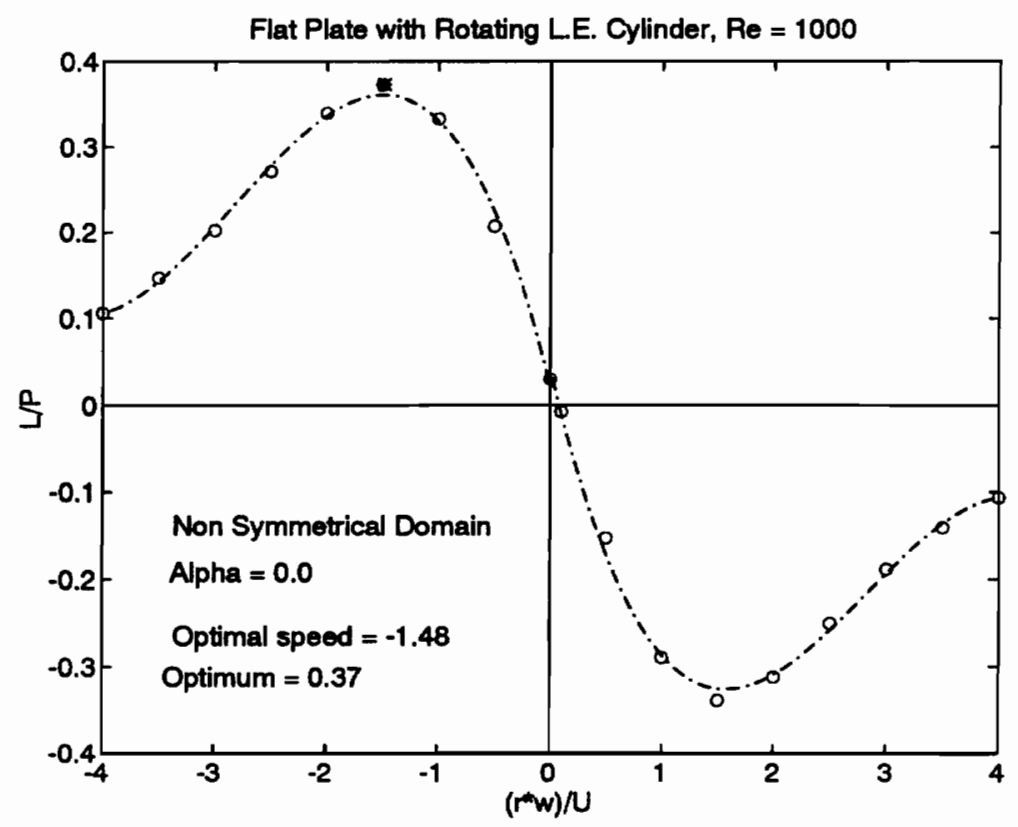

b) Symmetrical Domain

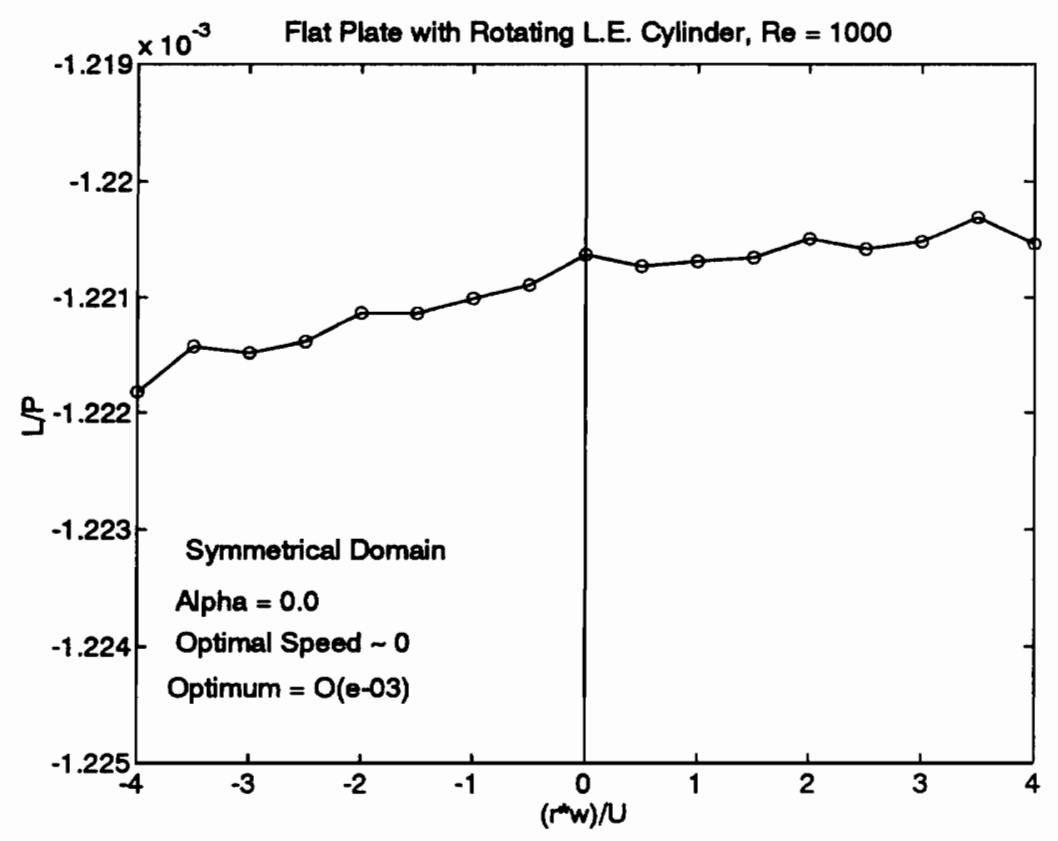

Figure 5.7 Optimization Problem, $\alpha=0^{\circ}$ 


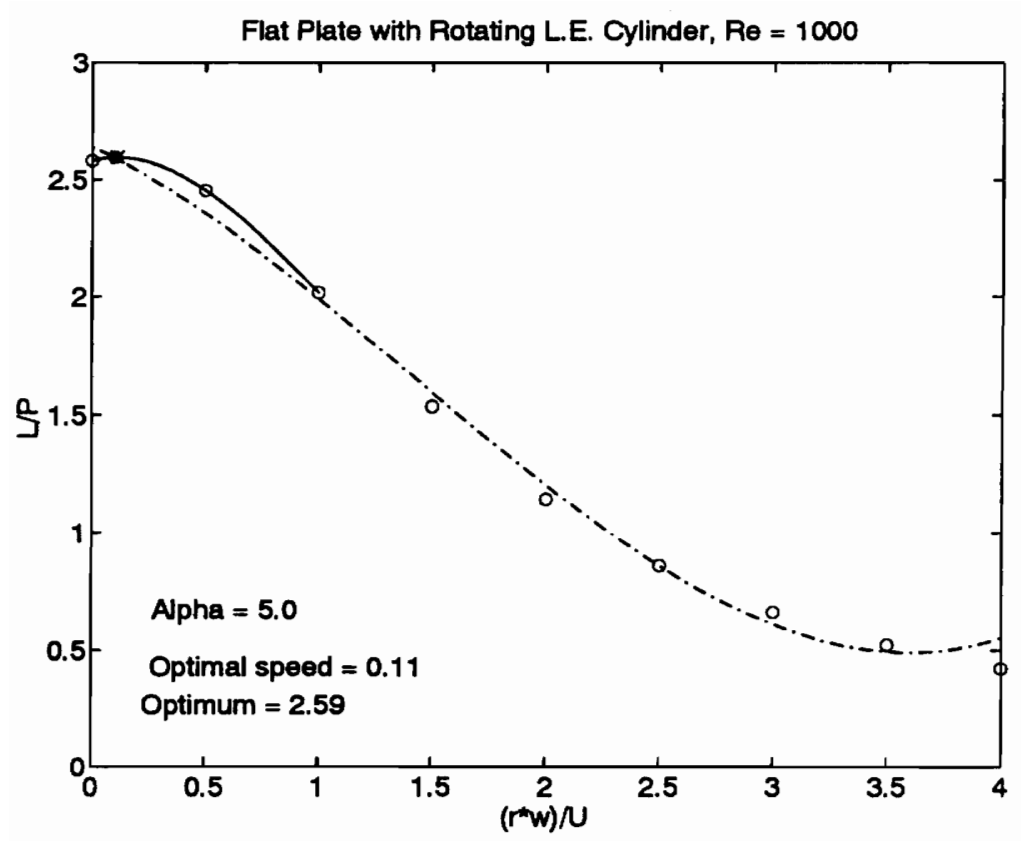

Figure 5.8 Optimization Problem, $\alpha=5^{\circ}$

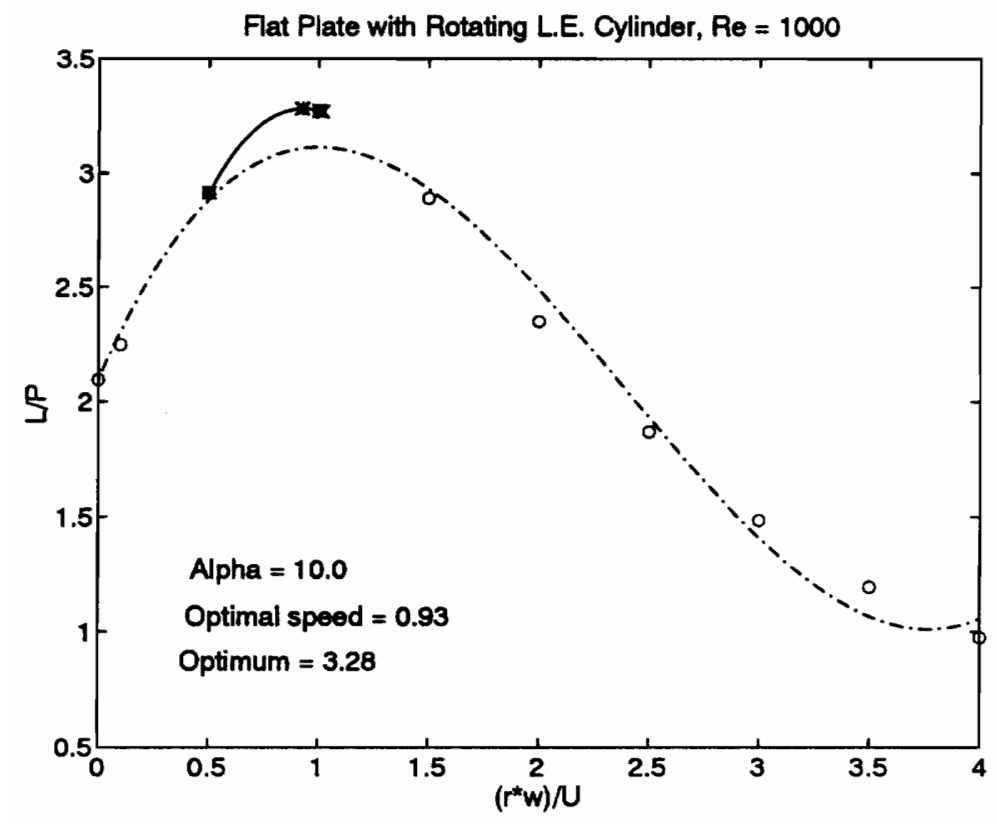

Figure 5.9 Optimization Problem, $\alpha=10^{\circ}$ 


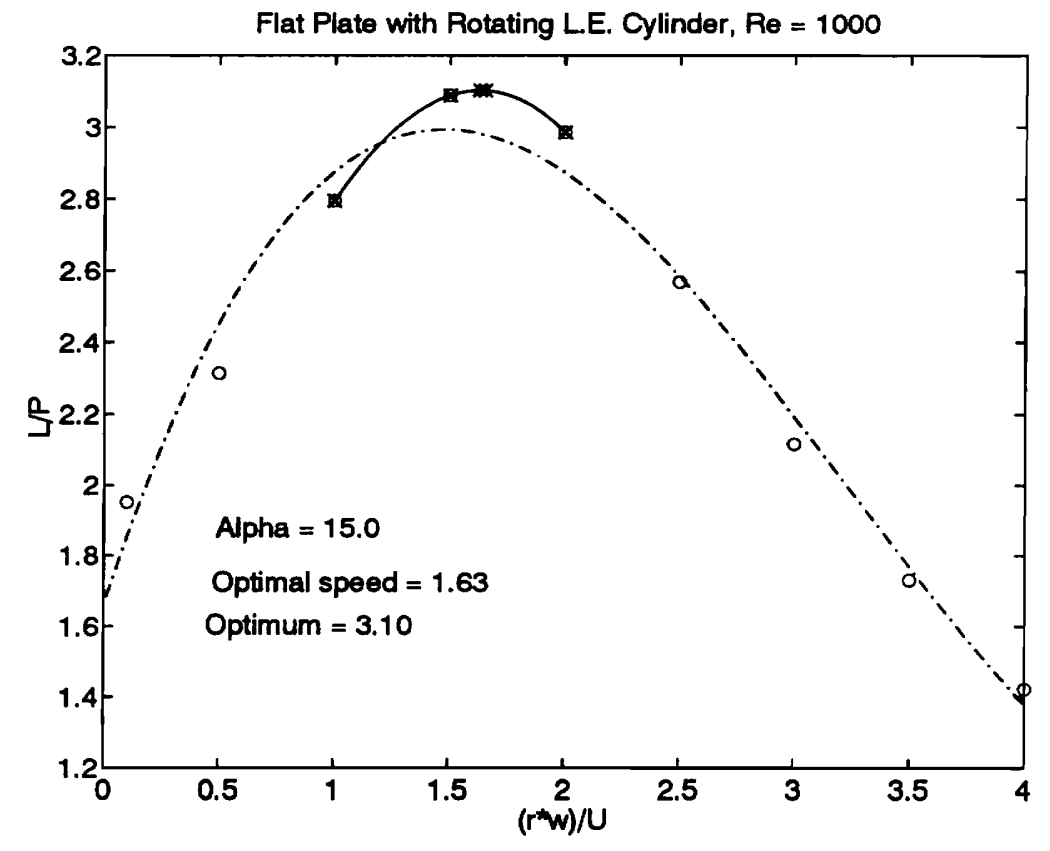

Figure 5.10 Optimization Problem, $\alpha=15^{\circ}$

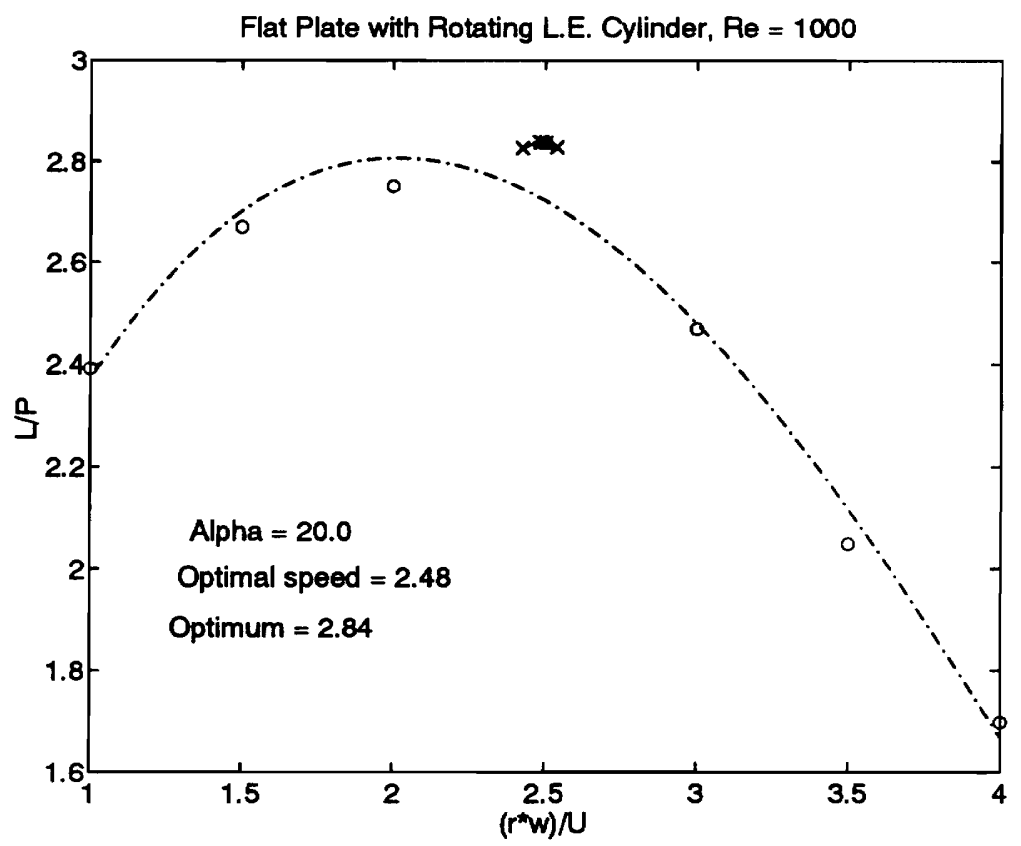

Figure 5.11 Optimization Problem, $\alpha=20^{\circ}$ 


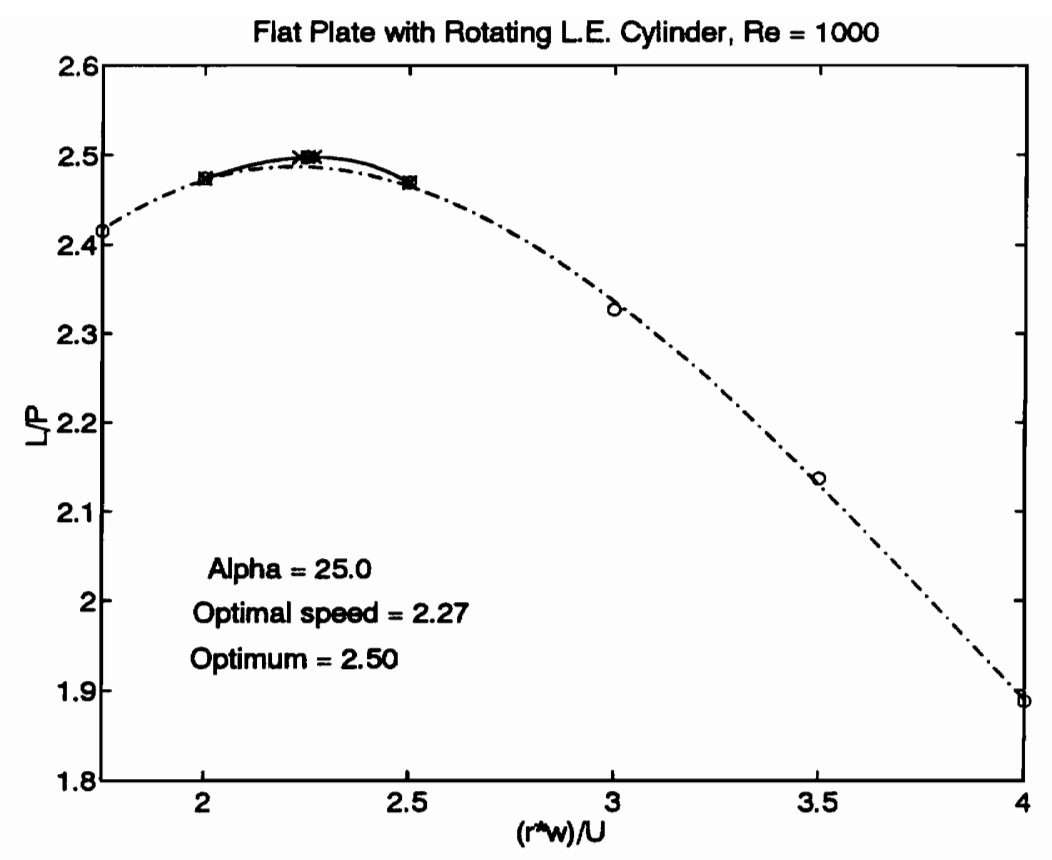

Figure 5.12 Optimization Problem, $\alpha=25^{\circ}$

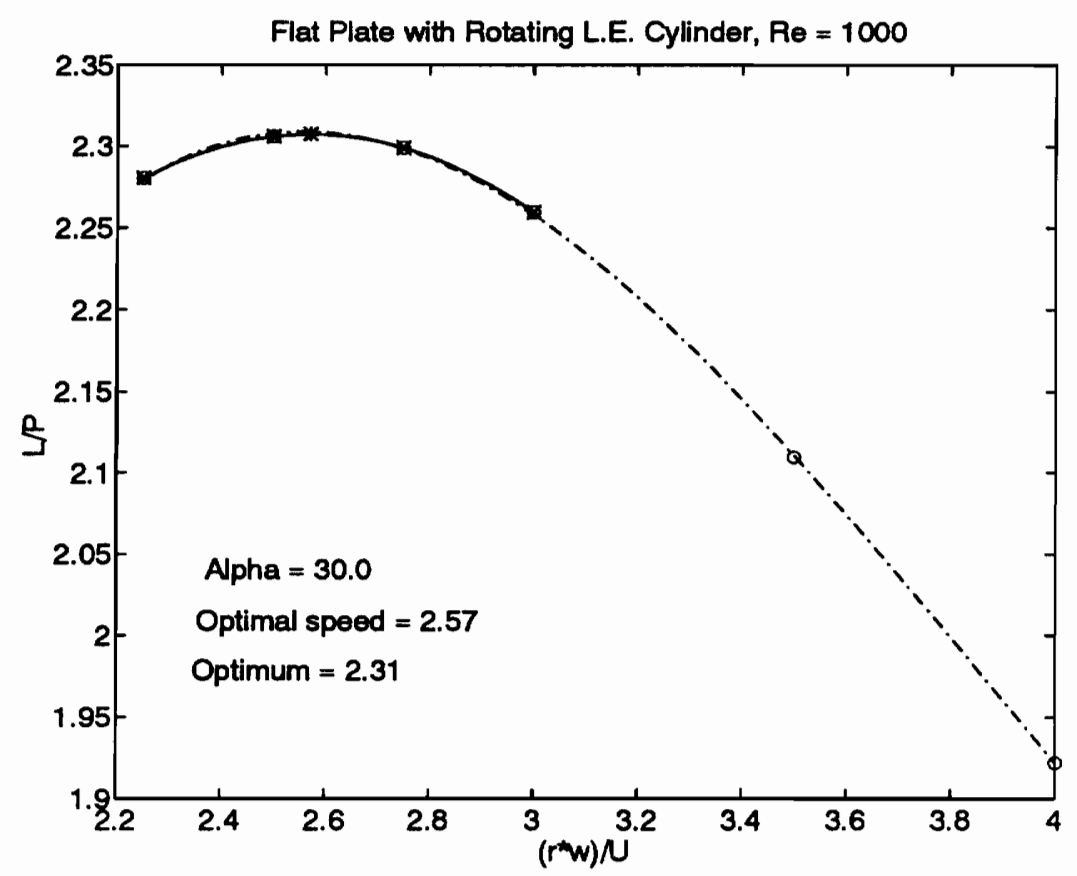

Figure 5.13 Optimization Problem, $\alpha=30^{\circ}$ 


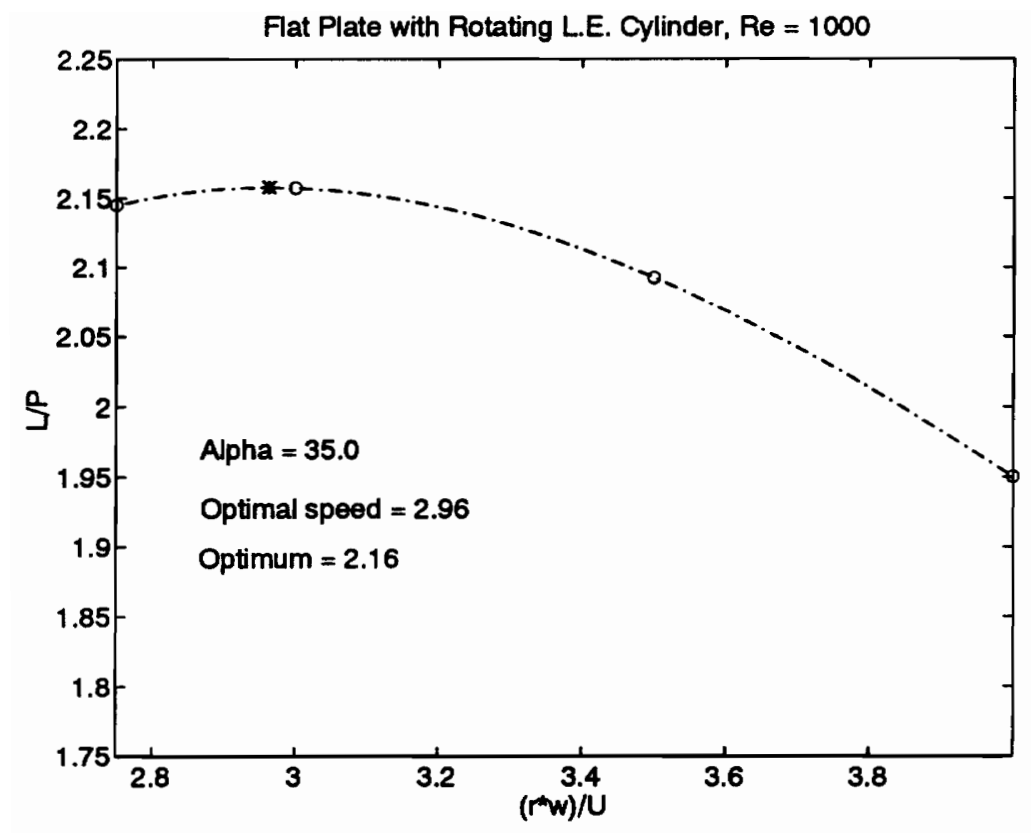

Figure 5.14 Optimization Problem, $\alpha=35^{\circ}$

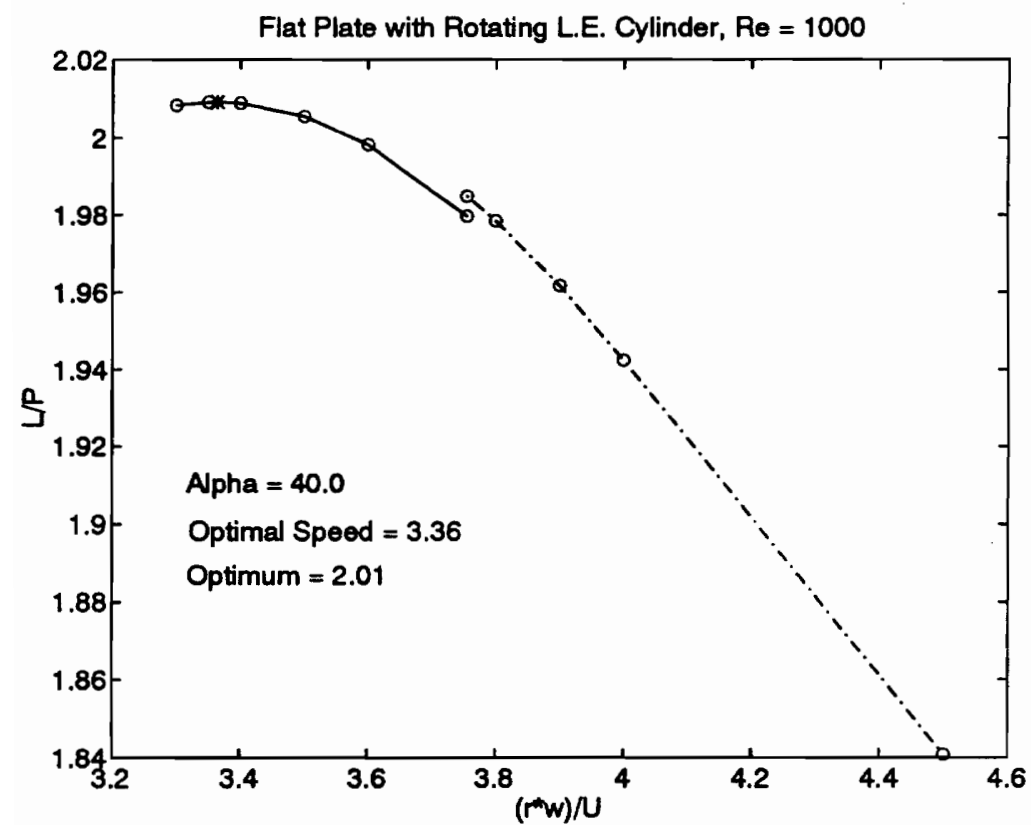

Figure 5.15 Optimization Problem, $\alpha=40^{\circ}$ 


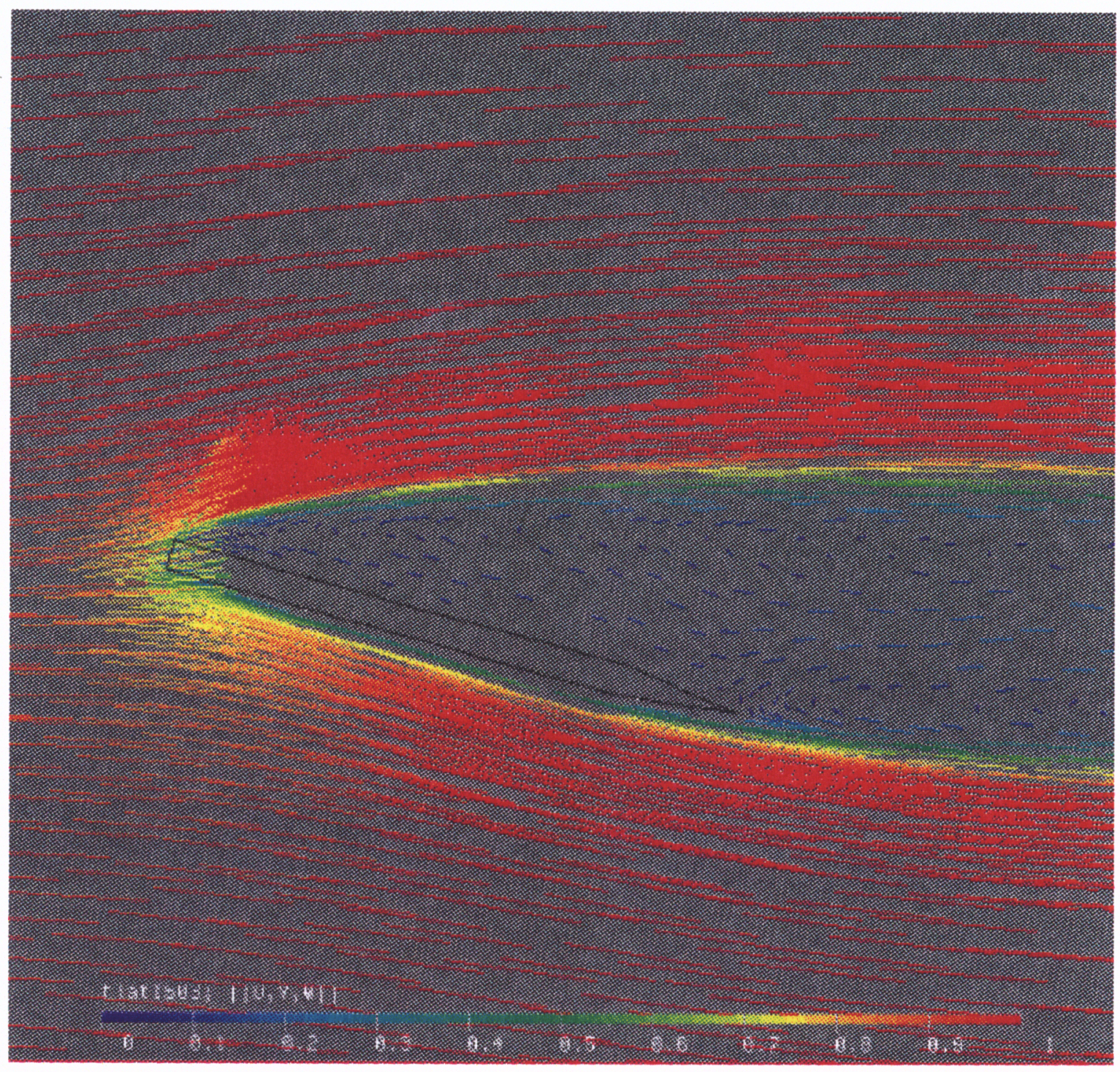

Figure 5.16 Base Flat Plate, Alpha = 15, Velocity Distribution 


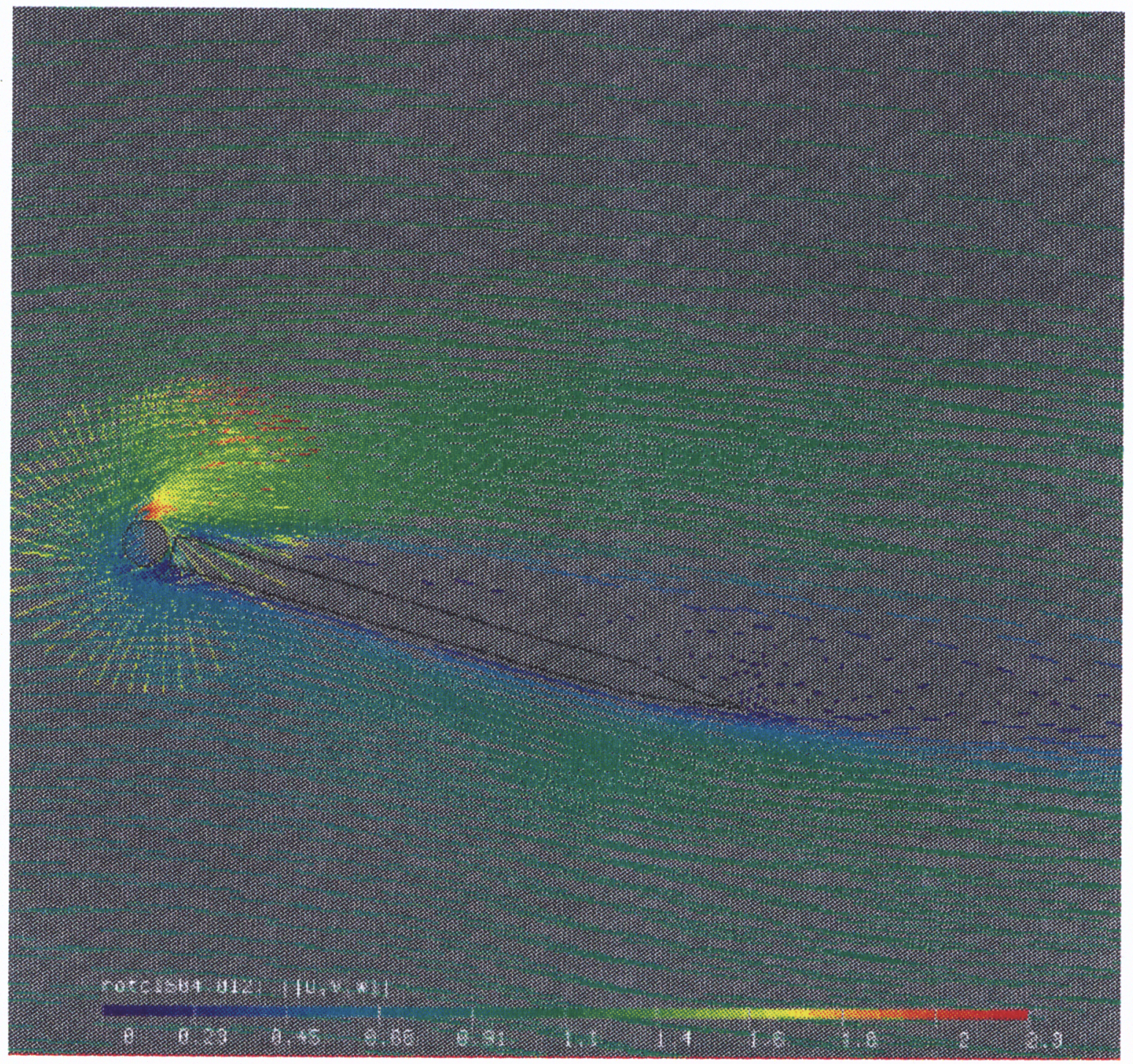

Figure 5.17 Flat Plate with Rotating L.E.

Cylinder, Alpha $=15,(r * w) / U=1.63$

= optimal speed 
Except for the special case of $\alpha=20^{\circ}$ discussed in section 4.5, no problem occured in the optimization process for the angles of attack from $5^{\circ}$ to $35^{\circ}$. On the other hand, both upper and lower limits (i.e. $\alpha=0^{\circ}$ and $\alpha=40^{\circ}$ ) needed special care in the treatment. First, we will discuss the zero angle of attack case.

The non symmetry in the $y$ direction of the overall domain presented in section 3.2.1 provoked unexpected results for $\alpha=0^{\circ}$ (see figure $5.7 \mathrm{a}$ )). The different amount of fluid on the upper and the lower side of the plate combined with the cylinder rotation induced non negligible fluctuations in the lift. This consequence combined with the rotational power effect produced large variations in the performance ratio. Because of the particular curve obtained over the feasible interval, we decided to investigate further and reverse the rotational sense of the cylinder. We obtained the graph presented in figure 5.7 a). The sign of the performance ratio became positive for negatives values of $\omega$ and the optimization process run on this curve resulted in an optimal speed of around -1.5 . When we changed the external boundaries in order to get a symmetrical domain, we obtained the curve shown in Figure 5.7 b).

This results is rather more physically acceptable. The fluctuations in the lift are practically eliminated and the rotational power effect has little influence on the performance ratio. Physical symmetry suggests that the graph should be symmetric about $\omega=0$. The grid generation procedure is not symmetrical, as can be seen in the annulus flow results of section 3.1.3. We believe the non-zero performance ratio is an artifact of the finite element grid and "claim" that $\omega=0$ is a reasonable optimal value for the cylinder rotational speed.

On the upper bound of the angle of attack interval $\left(\alpha=40^{\circ}\right)$, it was more difficult to obtain an optimization problem. At such a high angle of attack, the flow becomes very difficult to solve even with the cylinder rotating at high speed $\left(\left(r^{*} w\right) / U=4\right)$. In order to obtain a solution on the initial grid, we had to cut the Reynolds number by half and then increase the Reynolds number in steps to reach a thousand. Subsequently, we tried to produce a few surrounding data points for the objective function to locate a peek in the curve. Yet, at speed ratio smaller that 4 , the flow resolution becomes increasingly difficult to solve. We proceeded by slowly decreasing the cylinder angular speed until no solution could be obtained with the grid used. At that point, the performance ratio had increased little by little but had never gone down. With a slightly finer grid, we were able to decrease 
the cylinder rotational speed further in order to obtain a maximum for the performance ratio. We can observe in Figure 5.15, a discontinuity at the point were the grid changed. For the optimization problem, we only used the left portion of the curve, i.e. the finer grid calculations.

Figure 5.16 and 5.17 present respectively the flat plate alone and with a cylinder rotating at optimal speed for an angle of attack of $15^{\circ}$. The cylinder rotation reattaches the flow to the surface effectively to obtain maximal gain.

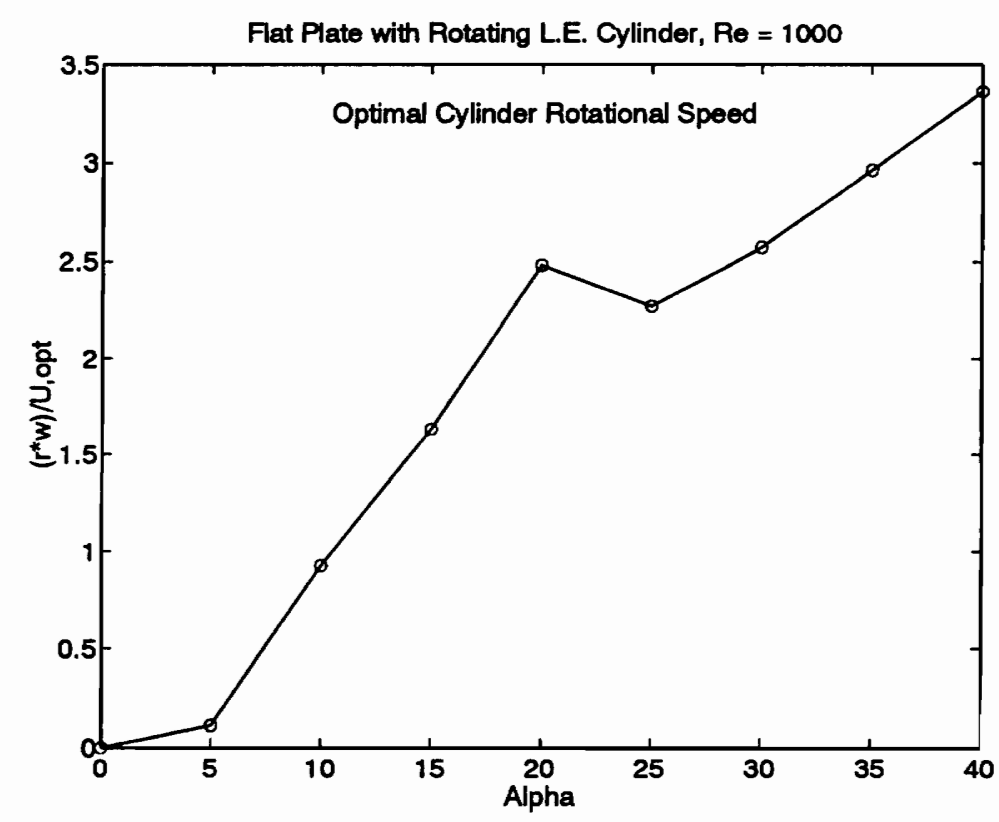

Figure 5.18 Optimal Cylinder Angular Speed versus Angle of Attack

Table 5.1 Optimization Results

\begin{tabular}{|c|c|c|c|c|}
\hline $\begin{array}{c}\text { Angle of } \\
\text { Attack }\end{array}$ & $\begin{array}{c}\text { Number } \\
\text { of } \\
\text { Iterations }\end{array}$ & $\begin{array}{c}\text { Optimal } \\
\text { Performance } \\
\text { Ratio }\end{array}$ & $\begin{array}{c}\text { Optimal } \\
\text { Angular Speed }\end{array}$ & Error \\
\hline \hline 0 & 0 & $-1.22 \mathrm{e}-03$ & 0.000 & O(e-03) \\
\hline 5 & 2 & 2.59399 & 0.11114 & $1.72 \mathrm{e}-05$ \\
\hline 10 & 4 & 3.28097 & 0.926646 & $9.57 \mathrm{e}-06$ \\
\hline 15 & 3 & 3.10177 & 1.62892 & $7.90 \mathrm{e}-06$ \\
\hline 20 & 6 & 2.83815 & 2.48180 & $3.64 \mathrm{e}-06$ \\
\hline 25 & 4 & 2.49773 & 2.26696 & $1.81 \mathrm{e}-05$ \\
\hline 30 & 2 & 2.30759 & 2.57091 & $9.90 \mathrm{e}-05$ \\
\hline 35 & 1 & 2.15770 & 2.96324 & $5.05 \mathrm{e}-05$ \\
\hline 40 & 1 & 2.00921 & 3.36385 & $2.46 \mathrm{e}-07$ \\
\hline
\end{tabular}


The final results for the optimization study are summarized in Figure 5.18 and Table 5.1. As we can observe by considering the performance ratio versus cylinder speed graphs in Figures 5.8 through 5.15 , the performance ratio peak gradually shifts to the right as the angle of attack increases. This observation is confirmed by Figure 5.18 and results from the growing adverse pressure gradient as the angle of attack increases. The small peak observed around $\alpha=20^{\circ}$ cannot be clearly explained. It may be caused by the separation phenomenon which occurs around this value or, recalling the problem mentioned in section 4.5 , it may be a weakness of the optimization algorithm. As for the possibility of a mathematical relation between the optimal cylinder rotational speed and the angle of attack, Johnson, Tennant and Stamps ${ }^{10}$ obtained a fairly linear relation between the two quantities. However, their study only covered the interval $0^{\circ}$ to $15^{\circ}$ angle of attack. Figure 5.18 presents a linear behavior in the same interval but it subsequently deteriorates after $20^{\circ}$ and becomes linear again between $25^{\circ}$ and $40^{\circ}$. In this sense, the optimal curve may be piecewise linear. 


\section{CHAPTER 6}

\section{CONCLUSION}

In the previous chapters, we presented an optimization procedure to determine the "best" rotational speed of a spinning leading edge cylinder acting as a high lift device for a flatplate airfoil model. We used an adaptive finite element method to solve the flow problem and compute the aerodynamic forces acting on the plate-cylinder system. This method has been proven effective in three different calibration examples studied in the third chapter.

The optimization problem has been stated as a simple unconstrained case, maximizing the lift to cost ratio. In the cost function, we added the power required to rotate the cylinder to form an extension of the usual lift to drag ratio. Previous investigations on the effect of this boundary-layer control method neglected this design consideration. The general conclusion drawn from all these experiments is that good aerodynamic performance can be obtained with this method and that higher rotational speeds are more effective than lower speeds. The inclusion of the required power to rotate the cylinder made possible the optimization problem by adding a penalty to the more powerful high rotational speeds. The optimization results show a gradual increase in the optimal rotational speed as the angle of attack goes up. An apparent linear relation between $5^{\circ}$ and $20^{\circ}$ for the graph of the optimal speed versus angle of attack confirms some observations made by Johnson, Tennant and Stamps ${ }^{10}$ for low angles of attack. However, this linear relation deteriorates past $20^{\circ}$ to become linear again between $25^{\circ}$ and $40^{\circ}$. This discontinuity might be caused by the separation phenomenon occuring around $20^{\circ}$.

This optimization study provides a reasonable portrayal of the behavior for a moving boundary-layer control on a lifting surface. However, some parameters could be changed or added to improve the realistic representation of the problem. For example, with a more powerful computer system, we could generate very fine grids and solve the flow at higher Reynolds numbers. Furthermore, we could replace the flat-plate model with a real airfoil and compare the numerical results to previous experiments in a quantitative way. Modifications to the optimization problem could be made in order to provide more details to the optimal design. In addition to the rotational speed of the cylinder, we could investigate other parameters such as the cylinder diameter, the airfoil-cylinder gap, and the location of the cylinder on the airfoil. With all of the pertinent design parameters 
considered, an ideal airfoil could be primarily designed with numerical analysis, greatly reducing time and resources in the total design process. 


\section{REFERENCES}

1. Von Kármán, T., Aerodynamics: Selected Topics in the Light of Their Historical Development, Cornell University Press, Ithaca, N.Y., 1954, chap. I \& II.

2. Anderson, J.D., Introduction to flight, McGraw-Hill, N.Y., 1978, pp. 129-133.

3. Gad-el-Hak, M., Flow Control, Appl. Mech. Rev. v.42, 1989, pp. 261-293.

4. Swanson, W.M., The Magus Effect: A Summary of Investigation to Date, Transaction of the ASME, Journal of Basic Engineering, v.83, Sept. 1961, pp. 461-470

5. Goldstein, S., Modern Developments in Fluid Dynamics, Clarendon Press, Oxford, England, 1938, pp. 545-549.

6. Alvarez-Calderon, A., Arnold, F.R., Rotating Cylinder Flaps for V/S.T.O.L. Aircraft, Aircraft Engineering, v.36, 1964, pp. 304-309.

7. Cook, W.L., Mickey, D.M., Quigley, H.G., Aerodynamics of Jet Flap and Rotating Cylinder Flap STOL Concept, AGARD Fluid Dynamic Panel on V/STOL Aerodynamics, Delft, Netherlands, April 1974, Paper No.10.

8. Rotating Cylinder Flaps Tested on OV-10A, Aviation Week and Space Technology, v.95, No.16, Oct. 1971, p.19 and No.24, Dec. 1971, cover page.

9. Cichy, D.R., Harris, J.W. and MacKay, J.K., Flight Tests of a Rotating Cylinder Flap on a North American Rockwell YOV-10A Aircraft, NASA CR-2135, Nov. 1972.

10. Jonhson, W.S., Tennant, J.S., and Stamps, R.E., Leading Edge Rotataing Cylinder for Boundary-Layer Control on Lifting Surfaces, Journal of Hydronautics, v.9, No.2, April 1975, pp. 76-78.

11. Tennant, J.S., Johnson, W.S., and Krothapalli, A., Rotating Cylinder for Circulation Control on an Airfoil, Journal of Hydronautics, v.11, No.3, July 1976, pp. 102-105.

12. Tennant, J.S., Johnson, W.S., and Keaton, D.D., Boundary-Layer Flows from Fixed to Moving Surfaces Including Gap Effects, Journal of Hydronautics, v.12, No.2, April 1978, pp. 81-84.

13. Mokhtarian, F., Modi, V.J., and Yokomizo, T., Effects of Moving Surfaces on the Airfoil Boundary-Layer Control, AIAA Atmospheric Flight Mechanics Conference, Minneapolis, Minnesota, August 1988. 
14. Modi, V.J., Sun, J.L.C., Akutsu, T., Lake, P. McMillian, K., Swinton, P.G., and Mullins, D., Moving Surface Boundary-Layer Control for Aircraft Operation at High Incidence, Journal of Aircraft, AIAA, v.18, No.11, Nov. 1981, pp. 963-968.

15. Mokhtarian, F., Modi, V.J., and Yokomiso, T., Rotating Air Scoop as Airfoil Boundary-Layer Control, Engineering Notes, v.25, No.10, Oct. 1988, pp. 973-975.

16. Modi, V.J., Mokhtarian, F., Fernando, M.S.U.K., Yokomizo, T., Moving Surface Boundary-Layer Control as Applied to Two-Dimensional Airfoils, AIAA 89-0296.

17. Mokhtarian, F., and Modi, V.J., Fluid Dynamics of Airfoils with Moving Surface Boundary-Layer Control, Journal of Aircraft, v.25, No.2, Feb. 1988, pp. 163-169.

18. Reddy, J.N., An Introduction to the Finite Element Method, McGraw-Hill, N.Y., 1984.

19. Hétu, J.-F., Méthodes d'Élements Finis Adaptatives pour les écoulements visqueux Incompressibles, $\mathrm{PhD}$ thesis, Applied Mathematics, École Polytechnique de Montréal, Dec. 1991.

20. Pelletier, and D.H., Hétu, J.-F., An Adaptative Finite Element Method for Thermal Hydraulics Problems, 31st Aerospace Sciences Meeting \& Exhibit, Jan. 11-14,1993, Reno, NV., AIAA 93-0920

21. Pelletier, D.H., Survey of Finite Elements for Incompressible Flows, M.Sc. thesis, Mathematics, Virginia Polytechnic Institute and State University, Oct. 1984.

22. Pelletier, and D.H., Hétu, J.-F., An Adaptative Finite Element Methodology for Incompressible Viscous Flow, Advances in Finite Element Analysis in Fluid Dynamics II, ASME Winter Annual Meeting, Anaheim CA, Nov. 8-13, 1992.

23. Gill, P.E., Murray, W., Wright, M.H., Practical Optimization, Academic Press, London, 1981, chap. $2 \& 3$.

24. Knudsen, J.G., Katz, D.L., Fluid Dynamics and Heat Transfer, McGraw-Hill, New York, 1958.

25. Burden, R.L., Faires, J.D., Numerical Analysis (Fourth Edition),PWS-KENT, Boston, 1989.

26. Caillé, J. and Schetz, J.A., Finite-Element Navier-Stokes Analysis of the Flow About a Finite Plate, AIAA Journal, v.27, No.8, Aug. 1989, pp. 1089-1096.

27. Walter, K.T. and Larsen, P.S., The FON Method for the Steady Two-Dimensional Navier-Stokes Equations, Computers and fluids, Vol. 9. No. 3, Sept. 1981, pp. 365-376. 
28. Modi, V.J., Fernando, M.S.U.K., Drag Reduction of Bluff Bodies Through Moving Surface Boundary Layer Control, AIAA-90-0298.

29. Peraire, J., Vahdati, M., Morgan, K., Zienkeiwicz, O.C., Adaptive Remeshing for Compressible Flow Computations, Journal of Computational Physics, Vol. 72, No. 2, pp. 26-37, 1987. 


\section{VITA}

The author was born in Montréal on August 28, 1968. She completed her Bachelor's degree in mechanical engineering at the École Polytechnique de Montréal in 1991. On summer internships from the National Research Council of Canada, she worked with Dr. D.H. Pelletier in 1990 and 1991 on computational fluid dynamics. With Dr. Pelletier's encouragement, she enrolled in the Aerospace and Ocean Engineering Department at VPI \& SU with a master scholarship from the government of Québec and an assistantship from VPI \& SU. She was a member of the International Development and Education Assembly (I.D.E.A.) under the International Club of VPI \& SU in 1992-1993 and participated in two projects with the Theater Department of Virginia Tech. She will work for the Interdisciplinary Center for Applied Mathematics during summer 1993. 